\title{
Modelos semiparamétricos com resposta binomial negativa
}

\author{
Fábio Hideto Oki
}

DisSERTAÇÃo APRESENTADA

AO

Instituto De MAtemÁtica e Estatística

DA

Universidade DE SÃo PAUlo

PARA

OBTENÇÃO DO TÍTULO

$\mathrm{DE}$

Mestre em CiÊnCIAS

\author{
Programa: Estatística \\ Orientador: Prof. Dr. Gilberto Alvarenga Paula
}

Durante o desenvolvimento deste trabalho o autor recebeu auxílio financeiro da CAPES

São Paulo, Maio de 2015 


\title{
Modelos semiparamétricos com resposta Binomial Negativa
}

\author{
Esta dissertação contém as correções e alterações \\ sugeridas pela Comissão Julgadora durante a defesa \\ realizada por Fábio Hideto Oki em 14/05/2014. \\ O original encontra-se disponível no Instituto de \\ Matemática e Estatística da Universidade de São Paulo.
}

Comissão Julgadora:

- Prof $^{\mathrm{a}}$. Dra . Gilberto Alvarenga Paula (orientador) - IME-USP

- Prof. Dr. Cristian Marcelo Villegas Lobos - ESALQ-USP

- Prof. Dr. Silvia Nagib Elian - IME-USP 


\section{Agradecimentos}

Agradeço meu orientador Dr. Gilberto Alvarenga Paula por toda sua dedicação e orientação que foram fundamentais para a realização deste trabalho.

Ao Instituto de Matemática e Estatística pela oportunidade para o meu crescimento profissional.

À CAPES que financiou este trabalho.

Aos meus familiares que apoiaram a minha vinda e continuaram incentivando por todo o período de realização deste trabalho.

Aos companheiros de pós-graduação que contribuíram com meu crescimento acadêmico. 


\section{Resumo}

O objetivo principal deste trabalho é discutir estimação e diagnóstico em modelos semiparamétricos com resposta binomial negativa, mais especificamente, modelos de regressão com resposta binomial negativa em que uma das variáveis explicativas contínuas é modelada de forma não paramétrica. Iniciamos o trabalho com um exemplo ilustrativo e fazemos uma breve revisão dos modelos paramétricos com resposta binomial negativa. Em seguida, introduzimos os modelos semiparamétricos com resposta binomial negativa e discutimos alguns aspectos de estimação, inferência e seleção de modelos. Dedicamos um capítulo a procedimentos de diagnóstico, tais como desenvolvimento de medidas de alavanca e de influência sob os aspectos de deleção de pontos e influência local, além de abordar a análise de resíduos. Reanalizamos o exemplo ilustrativo sob o enfoque semiparamétrico e apresentamos algumas conclusões.

Palavras-chave: dados de contagem, distância de Cook, distribuição binomial negativa, influência local, métodos não paramétricos, splines cúbicos. 


\section{Abstract}

The aim of this work is to discuss some aspects on estimation and diagnostics in negative binomial regression models which an explanatory continuous variable is modeled nonparametrically. First, an illustrative example is presented and analyzed under parametric negative binomial regression models. The proposed models are then introduced and some aspects on estimations, inference and model selection are presented. Particular emphasis is given on the development of diagnostic procedures, such as leverage measures, Cook distances, local influence approach and residuals. The motivated example is reanalyzed under the semiparametric viewpoint and some conclusions are given.

Keywords: count data, Cook distance, cubic splines, local influence, negative binomial distribution, nonparametric methods. 


\section{Sumário}

1 Introdução $\quad 1$

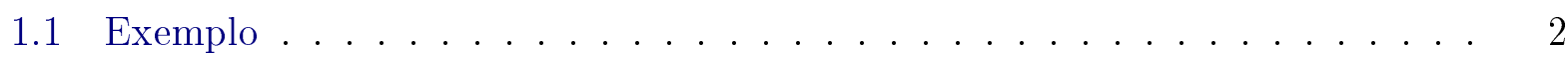

1.2 Organização do Trabalho . . . . . . . . . . . . . . . . . . 9

2 Revisão dos modelos paramétricos com resposta binomial negativa $\quad 10$

2.1 Distribuição binomial negativa . . . . . . . . . . . . . . . . . 10

2.2 Modelos com resposta binomial negativa . . . . . . . . . . . . . 11

2.3 Qualidade do ajuste . . . . . . . . . . . . . . . 14

2.4 Técnicas de diagnóstico . . . . . . . . . . . . . . . . 15

2.5 Seleção de modelos . . . . . . . . . . . . . . . . . . 16

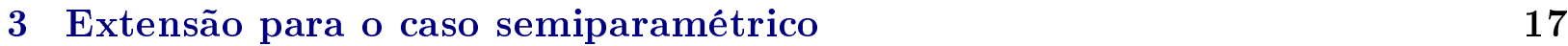

3.1 Especificação do modelo . . . . . . . . . . . . . . . . . . 17

3.2 Método de suavização spline cúbico . . . . . . . . . . . . . . . . . 18

3.3 Critério de penalização . . . . . . . . . . . . . . . . 20

3.3 .1 Função de penalização . . . . . . . . . . . . . . . . . 21

3.3.2 Logaritmo da função de verossimilhança penalizada . . . . . . . . . . 22

3.3.3 Função escore e informação de Fisher penalizadas . . . . . . . . . . . 23

3.4 Estimação dos parâmetros . . . . . . . . . . . . . . . . . . 25

3.4.1 Graus de liberdade efetivos . . . . . . . . . . . . . 27

3.4 .2 Estimação do parâmetro de suavização . . . . . . . . . . . . 28

3.5 Resultados assintóticos . . . . . . . . . . . . . . . . . 28

3.6 Considerações . . . . . . . . . . . . . . . . . . . . . . . 29

4 Métodos de diagnóstico $\quad 30$

4.1 Matrizes de alavancas . . . . . . . . . . . . . . . . . . . . 31

4.2 Resíduos . . . . . . . . . . . . . . . . . . . . . . 32

4.3 Distância de Cook . . . . . . . . . . . . . . . . . . 32

4.4 Graus de liberdade efetivos . . . . . . . . . . . . . . . . . . 34

4.5 Influência local . . . . . . . . . . . . . . . . . . . . . 34

4.5.1 Ponderação de casos . . . . . . . . . . . . . . 35

4.5.2 Perturbação aditiva em uma variável explicativa contínua . . . . . . . 36 
4.6 Considerações . . . . . . . . . . . . . . . . . . 37

5 Aplicação $\quad 38$

6 Conclusões $\quad 45$

6.1 Considerações Finais . . . . . . . . . . . . . . . . . 45

$\begin{array}{ll}\text { A Gráfico da variável adicionada } & 46\end{array}$

$\begin{array}{ll}\text { B Derivações do MBNS } & 48\end{array}$

B.1 Funções escore penalizadas . . . . . . . . . . . . . . . . . . 48

B.2 Informação de Fisher penalizadas . . . . . . . . . . . . . . 50

C Diagnóstico $\quad 55$

C.1 Ponderação de casos . . . . . . . . . . . . . . . . 55

C.2 Pertubação aditiva em uma variável contínua do modelo . . . . . . . . . 56

D Códigos em R $\quad 59$

D.1 Ajuste do modelo via gamlss . . . . . . . . . . . . . . . . . 59

D.2 Gráfico envelope para o MSRBN ajustado via gamlss . . . . . . . . . . . 59

D.3 Gráficos de diagnóstico para o MSRBN ajustado via gamlss . . . . . . . . . . 61

$\begin{array}{ll}\text { Referências Bibliográficas } & 65\end{array}$ 


\section{Capítulo 1}

\section{Introdução}

O tema para este trabalho foi escolhido pela vasta aplicação dos modelos semiparamétricos na análise estatística e pela pouca disponibilidade de metodologias estatísticas para avaliar a adequação desse tipo de modelo. Mais especificamente, o estudo é direcionado para a análise de dados com resposta binomial negativa em que se tem uma variável explicativa com comportamento não paramétrico em relação à resposta.

O modelo de regressão com resposta binomial negativa, para dados de contagem, é comumente aplicado quando é verificado o fenômeno de sobredispersão. Este fenômeno ocorre quando é esperada uma distribuição de Poisson para a resposta, porém a variância é maior do que a resposta média. Uma causa provável desse fenômeno é a heterogeneidade das unidades amostrais que pode ser devido às variabilidades interunidades experimentais.

Como é comum algumas variáveis explicativas apresentarem comportamentos não lineares, ou seja, comportamentos que não têm formas definidas ou conhecidas, então com o intuito de obter um ajuste mais flexível para os dados utilizamos funções não paramétricas, que tiveram origem no calculo numérico. Temos na literatura vários tipos de funções não paramétricas, dentre os quais médias móveis, kernel, lowess, splines de suavização e abordagem por funções base, por exemplo os B-splines, que são todas técnicas de suavização para funções.

Para este trabalho utilizamos os splines cúbicos que são adaptativos e alcançam uma aproximação adequada para qualquer comportamento não linear, além do ganho computacional ser maior.

A inserção de uma função não paramétrica no modelo requer, além de análises descritivas usuais, análises de diagnóstico para avaliar a adequação do modelo, as quais não necessariamente coincidem com as análises aplicadas em modelos paramétricos com resposta binomial negativa.

O objetivo deste trabalho é desenvolver a teoria referente ao modelo de regressão com resposta binomial negativa na situação em que consideramos uma variável explicativa de comportamento não paramétrico e outras de comportamento paramétrico. Metodologias para avaliar a adequação do modelo bem como a sensibilidade das estimativas via análise de 
diagnóstico, conjuntamente com a implementação computacional no $\mathrm{R}$ de modelos ajustados via pacote gamlss no $R$, são apresentadas.

\subsection{Exemplo}

Vamos considerar, como exemplo motivacional, uma breve aplicação em que a máxima diária da concentração média de ozônio por hora (em ppm) em Los Angeles é ajustada através de diferentes modelos para dados de contagem.

Os dados utilizados para ilustração referem-se ao estudo da relação entre a concentração de ozônio na atmosfera (variável resposta) e outras variáveis meteorológicas (variáveis explicativas) na Bacia de Los Angeles em 1976, obtidos de Faraway (2006, Cap. 12). As variáveis consideradas são descritas abaixo:

- O3: máxima diária da concentração média de ozônio por hora em Upland, CA, medidas em partes por milhão (ppm);

- vh: altura da pressão 500 millibar, medida na base da força aérea de Vandenberg;

- wind: velocidade do vento em mph no aeroporto LAX;

- humidity: umidade em porcentagem no LAX;

- temp: temperatura na base aérea de Sandburg em graus Fahrenheit;

- ibh: altura da inversão da temperatura base em pés;

- dpg: gradiente da pressão a partir de Los Angeles para Daggert em mmHg;

- ibt: inversão da temperatura base no LAX em graus Fahrenheit;

- vis: visibilidade no LAX em milhas;

- day: dia do ano.

A variável resposta $\mathrm{O} 3$ bem como as 9 variáveis explicativas foram observadas durante 330 dias do ano de 1976. Para começarmos a análise de dados, vamos verificar os gráficos de dispersão com as respectivas curvas de suavização ajustadas.

A Figura 1.1 descreve os gráfios de dispersão entre a variável resposta O3 e as demais variáveis explicativas. Observamos em geral relações não lineares entre a variável resposta e as variáveis explicativas em estudo. Podemos notar, em particular, que a variável day é uma possível variável que pode estar se relacionando de forma não linear com O3.

Similarmente a Faraway (2006, Cap. 12) ignoramos uma possível correlação temporal para O3 e propomos inicialmente um modelo log-linear de Poisson com efeitos principais completo, ou seja, todas as variáveis explicativas descritas acima foram inseridas de forma linear no modelo. Prosseguimos a análise com a seleção das variáveis explicativas através 


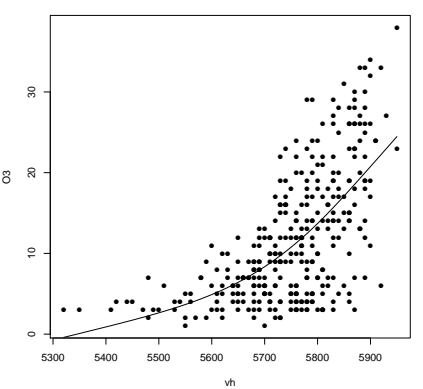

(a) $\mathrm{vh}$

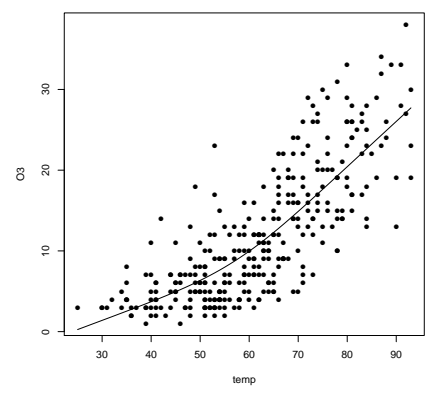

(d) temp

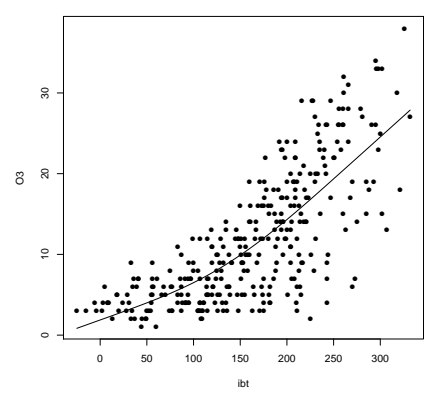

(g) ibt

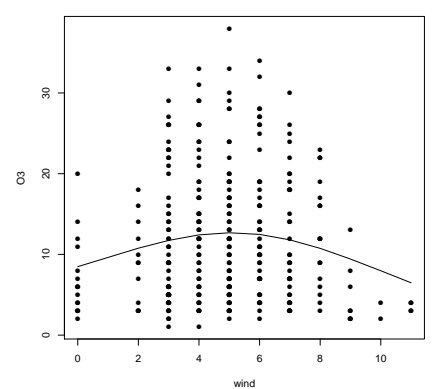

(b) wind

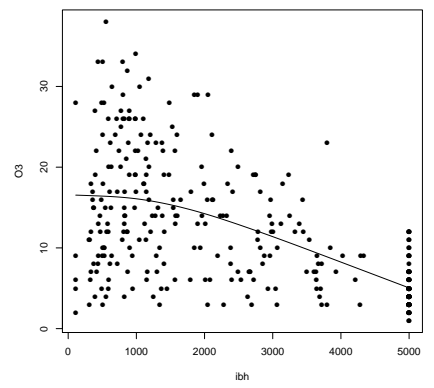

(e) ibh

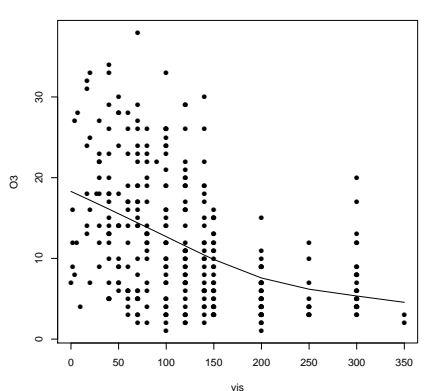

(h) vis

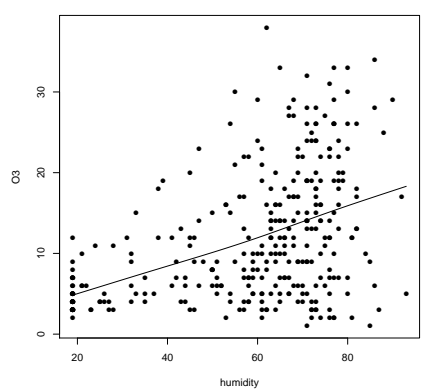

(c) humidity

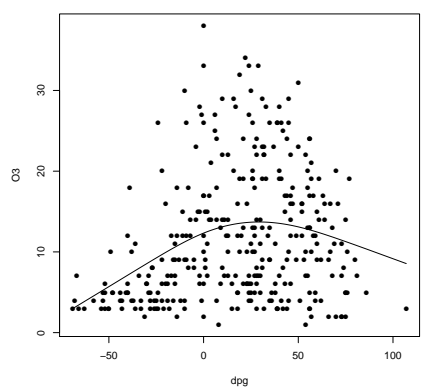

(f) dpg

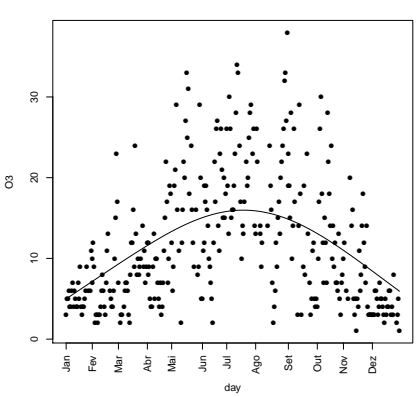

(i) day

Figura 1.1: Diagramas de dispersão entre a variável resposta $О 3$ e 9 variáveis explicativas meteorológicas observadas na bacia de Los Angeles em 1976. 
da metodologia de Akaike e posteriormente retiramos do modelo as variáveis explicativas que não foram marginalmente significativas ao nível de 5\%. Com isso, obtemos o seguinte modelo:

$$
\begin{aligned}
\text { O3 } & \sim \mathrm{P}\left(\mu_{i}\right) \\
\log \left(\mu_{i}\right) & =\beta_{0}+\beta_{1} \text { humidity }_{i}+\beta_{2} \text { temp }_{i}+\beta_{3} \mathrm{ibh}_{i}+\beta_{4} \mathrm{ibt}_{i}+\beta_{5} \operatorname{vis}_{i}+\beta_{6} \text { day }_{i}
\end{aligned}
$$

para $i=1, \ldots, 330$, em que $\mu_{i}$ representa a média $(\mathrm{ppm})$ de ozônio e $\boldsymbol{\beta}=\left(\beta_{0} ; \beta_{1} ; \ldots ; \beta_{6}\right)^{T}$ representa o vetor de parâmetros do modelo.

Os parâmetros do modelo foram estimados via máxima verossimilhança utilizando o software R (R Core Team, 2013) e estão descritas na Tabela 1.1.

Tabela 1.1: Estimativas de máxima verossimilhança com os respectivos erros padrão aproximados referentes ao modelo (1.1) ajustado aos dados de ozônio.

\begin{tabular}{crrr}
\hline Efeito & Estimativa & E. Padrão & valor $\mathbf{z}$ \\
\hline Intercepto & 0,616 & 0,131 & 4,698 \\
humidity & 0,008 & 0,001 & 7,217 \\
temp & 0,022 & 0,003 & 7,698 \\
ibh & $-6 \times 10^{-5}$ & $2 \times 10^{-5}$ & $-3,472$ \\
ibt & 0,002 & $7 \times 10^{-4}$ & 2,437 \\
vis & $-0,001$ & $3 \times 10^{-4}$ & $-2,594$ \\
day & $-0,001$ & $2 \times 10^{-4}$ & $-4,155$ \\
\hline
\end{tabular}

Nota-se pela Tabela 1.1 que todos os coeficientes são marginalmente significativos. À medida que aumenta a umidade, a temperatura ou a inversão de temperatura espera-se aumento para a concentração de ozônio, mantidas as demais variáveis explicativas fixas. Por outro lado, à medida que aumenta a altura da inversão da temperatura ou a visibilidade espera-se redução da concentração de ozônio, mantidas as demais variáveis explicativas fixas. A variável day não tem uma interpretação inferencial para a variável resposta no modelo. Estamos considerando day como uma variável controle, ou seja, uma variável de ajuste para o modelo proposto. Observando a Figura 1.1(i) nota-se que há um crescimento da concentração de ozônio de Janeiro a Julho com um decrescimento até Dezembro. Todavia, o modelo indica para um decrescimento.Talvez a inclusão de um termo quadrático em day ou uma função não paramétrica em day contemplem melhor o comportamento dessa variável ao longo do tempo.

No entanto, quando verificamos a adequação do modelo pela análise de diagnóstico temos que o mesmo não está bem ajustado, como pode ser observada pela Figura 1.2. Essa figura descreve o gráfico normal de probabilidades para o resíduo componente do desvio e apresenta indícios de que há um afastamento da suposição de que a concentração de ozônio em ppm siga a distribuição de Poisson. O gráfico ainda sugere um fenômeno conhecido como sobredispersão. 


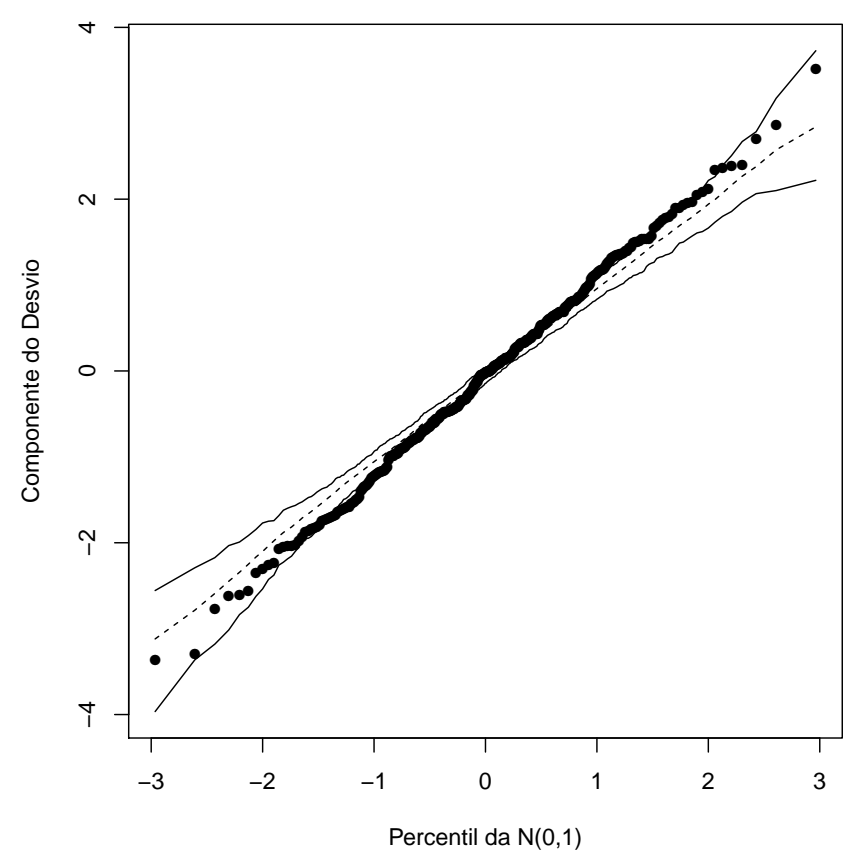

Figura 1.2: Gráfico normal de probabilidades com banda de confiança gerada de $95 \%$ para o resíduo componente do desvio referente ao modelo (1.1) ajustado aos dados de ozônio.

A banda de confiança apresentada na Figura 1.2 é conhecida na literatura como envelope e é construída através de simulações da distribuição de Poisson. Para mais detalhes o texto de Paula (2013) pode ser consultado.

A fim de tentarmos acomodar a sobredispersão observada na Figura 1.2, vamos propor o modelo log-linear com resposta binomial negativa com efeitos principais completo. Procedendo da mesma forma anterior, ou seja, fazendo a seleção de variáveis explicativas através do método de Akaike e posteriormente verificando quais variáveis não são marginalmente significativas ao nível $5 \%$, obtemos o seguinte modelo:

$$
\begin{aligned}
\text { O3 } & \sim \mathrm{BN}\left(\mu_{i}, \phi\right) \\
\log \left(\mu_{i}\right) & =\beta_{0}+\beta_{1} \text { humidity }_{i}+\beta_{2} \mathrm{temp}_{i}+\beta_{3} \mathrm{ibh}_{i}+\beta_{4} \mathrm{ibt}_{i}+\beta_{5} \operatorname{vis}_{i}+\beta_{6} \text { day }_{i}
\end{aligned}
$$

para $i=1, \ldots, 330$, em que $\mu_{i}$ representa a média (ppm) de ozônio, $\phi$ é o parâmetro de forma, e $\boldsymbol{\beta}=\left(\beta_{0} ; \beta_{1} ; \beta_{2} ; \ldots ; \beta_{6}\right)^{T}$ representa o vetor de parâmetros do modelo.

Os parâmetros do modelo foram estimados via máxima verossimilhança utilizando o software R (R Core Team, 2013) e estão descritas na Tabela 1.2.

Nota-se pela Tabela 1.2 que todos os coeficientes são marginalmente significativos. À medida que aumenta a umidade, a temperatura ou a inversão de temperatura espera-se aumento para a concentração de ozônio, mantidas as demais variáveis explicativas fixas. Por outro lado, à medida que aumenta a altura da inversão da temperatura ou a visibilidade 
Tabela 1.2: Estimativas de máxima verossimilhança com os respectivos erros padrão aproximados referentes ao modelo (1.2) ajustado aos dados de ozônio.

\begin{tabular}{crrr}
\hline Efeito & Estimativa & E. Padrão & valor $\mathbf{z}$ \\
\hline Intercepto & 0,612 & 0,150 & 4,085 \\
humidity & 0,008 & 0,001 & 6,087 \\
temp & 0,022 & 0,003 & 6,826 \\
ibh & $-6 \times 10^{-5}$ & $2 \times 10^{-5}$ & $-3,024$ \\
ibt & 0,002 & $8 \times 10^{-4}$ & 1,992 \\
vis & $-8 \times 10^{-4}$ & $3 \times 10^{-4}$ & $-2,423$ \\
day & $-9 \times 10^{-4}$ & $2 \times 10^{-4}$ & $-3,782$ \\
$\phi$ & 38,6453 & 12,9 & \\
\hline
\end{tabular}

espera-se redução da concentração de ozônio, mantidas as demais variáveis explicativas fixas. Novamente a variável day tem o mesmo intuito do modelo (1.1).

Verificamos a adequação do modelo pela análise de diagnóstico através do gráfico normal de probabilidade e gráficos de índices da distância de Cook .

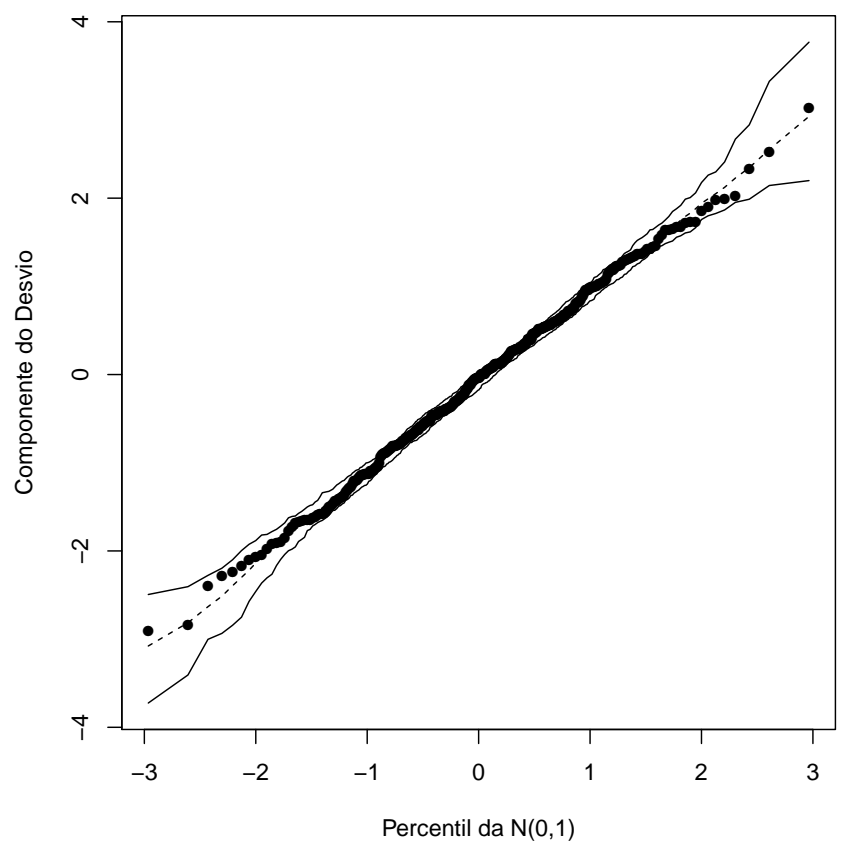

Figura 1.3: Gráfico normal de probabilidades com banda de confiança gerada de $95 \%$ para o resíduo componente do desvio referente ao modelo (1.2) ajustado aos dados de ozônio.

A Figura 1.3 apresenta o gráfico normal de probabilidades para o resíduo componente do desvio e não apresenta indícios de afastamentos da suposição de que a concentração de ozônio em ppm siga a distribuição binomial negativa.

A Figura 1.4 descreve o gráfico de índices da distâncias de Cook. Nota-se alguns possíveis pontos influentes no ajuste realizado, porém aplicando o refinamento das análises com a retirada dos pontos um por vez e refazendo o ajuste do modelo (1.2), percebemos que não 


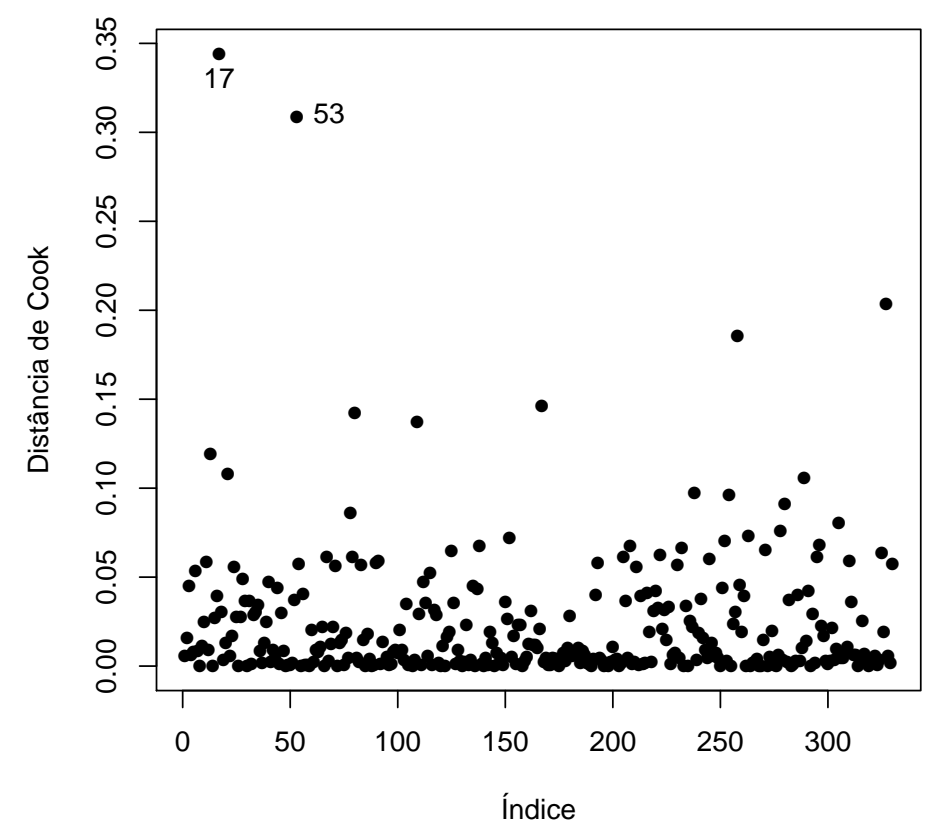

Figura 1.4: Gráfico de indices da distância de Cook referente ao modelo (1.2) ajustado aos dados de ozônio.

houve mudanças inferenciais. Neste caso, podemos dizer que não há indícios de sensibilidade da estimativa $\hat{\boldsymbol{\beta}}$ com a retirada individual das observações.

No entanto, a fim de verificarmos se podemos ter um ajuste melhor, construímos para cada uma das variáveis explicativas do modelo (1.2) o gráfico da variável adicionada.

A Figura 1.5 ilustra o gráfico da variável adicionada construído para as variáveis explicativas do modelo (1.2) com suas respectivas curvas de suavização. Esse tipo de gráfico sugere se alguma variável explicativa deve entrar no modelo além da forma linear e deve ser verificado através da linearidade ou não entre dois tipos de resíduos. Mais detalhes sobre o gráfico da variável adicionada podem ser encontrados no Apêndice A.

Neste caso, observamos que o gráfico sugere a inserção das variáveis explicativas humidity e day de forma não linear no modelo (1.2). Porém, podemos ignorar a não linearidade para a variável explicativa humidity, por estar suave. Já pelo gráfico da variável adicionada para a variável explicativa day, nota-se que o grau da curva de suavização é mais acentuado, indicando então que day deve entrar no modelo de forma não linear.

Assim, uma alternativa ao modelo proposto em (1.2) é tentar ajustar a variável day de forma não paramétrica através, por exemplo, de splines cúbicos, uma vez que este tipo de modelo não exige uma relação linear entre a variável resposta e a variável explicativa podendo produzir melhores ajustes.

Estudaremos, então, modelos que consideram a inclusão de uma variável explicativa na forma não paramétrica e retornaremos a este exemplo motivacional no final do trabalho assumindo que a variável day é considerada na forma não paramétrica. Comparações serão 


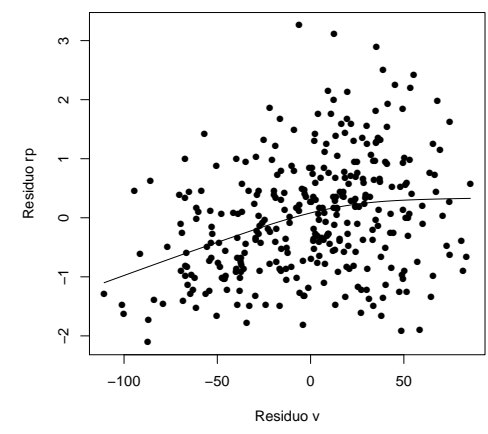

(a) humidity

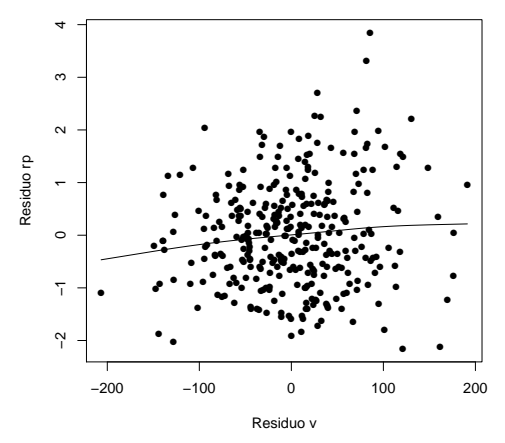

(d) ibt

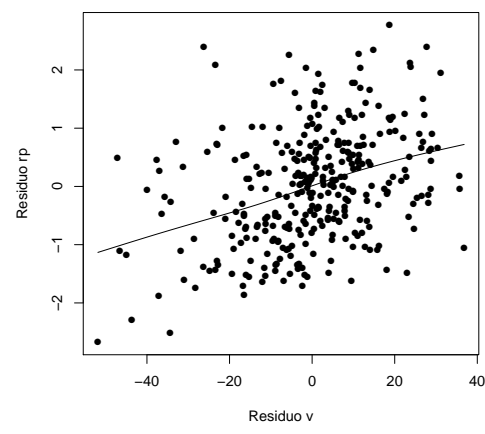

(b) temp

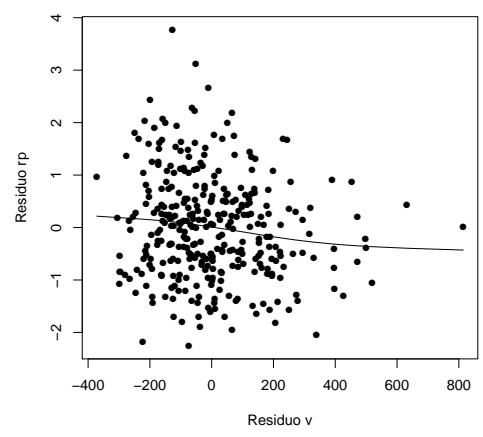

(e) vis

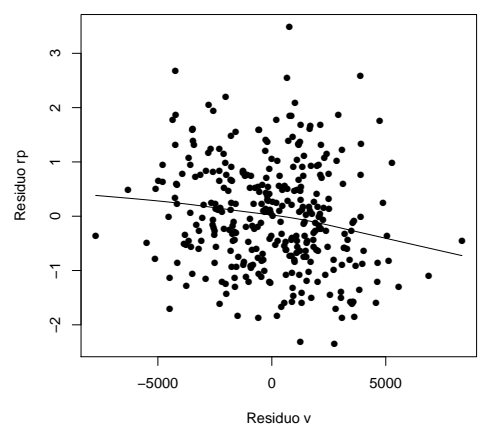

(c) ibh

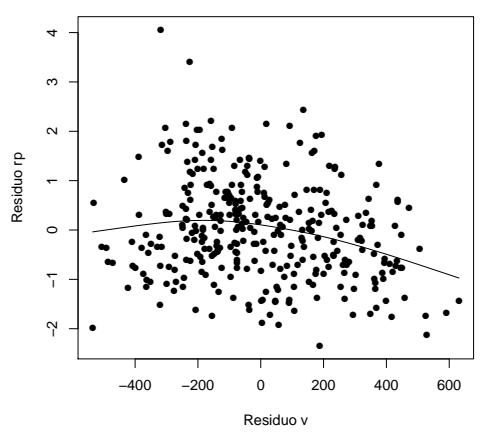

(f) $d a y$

Figura 1.5: Gráficos da variável adicionada referentes às variáveis explicativas do modelo (1.2) ajustado aos dados de ozônio. 
feitas com os resultados dos ajustes paramétricos apresentados neste capítulo.

\subsection{Organização do Trabalho}

No Capítulo 2 fazemos uma breve revisão do modelo com resposta binomial negativa, ou seja, revisamos conceitos básicos que envolvem o modelo em questão tais como construção do modelo, estimação dos parâmetros, qualidade do ajuste e técnicas de diagnóstico.

No Capítulo 3 descrevemos os modelos com variáveis explicativas paramétricas e uma variável explicativa não paramétrica, que serão denominados modelos semiparamétricos com resposta binomial negativa. Introduzimos aqui conceitos de especificações das variáveis, de ajuste via splines cúbicos e do parâmetro de suavização, além de definir a função de verossimilhança penalizada e os graus de liberdade efetivos. Integram ainda este capítulo a estimação dos parâmetros através da maximização da função de verossimilhança penalizada e desenvolvimento do método iterativo backfitting, estudo dos graus de liberdade efetivos, estimação do parâmetro de dispersão e suavização.

O Capítulo 4 resume técnicas de diagnóstico para o modelo apresentado no Capítulo 3 que permitam a análise de pontos de alavanca e influentes no componente paramétrico, no componente não paramétrico e na média.

Já no Capítulo 5 toda a teoria desenvolvida no trabalho é aplicada aos dados descritos em Faraway (2006, Cap. 12), em que a concentração de ozônio na atmosfera (variável resposta) é relacionada com outras variáveis meteorológicas (variáveis explicativas) na bacia de Los Angeles em 1976. No Capítulo 6 discutimos algumas conclusões obtidas neste trabalho analisando as vantagens e desvantagens do método proposto.

Toda parte computacional desenvolvidos ao longo desta dissertação para estimação, diagnóstico dos modelos e gráficos foram desenvolvidos utilizando o software $\mathrm{R}$ (disponível gratuitamente em http://r-project.org/). 


\section{Capítulo 2}

\section{Revisão dos modelos paramétricos com resposta binomial negativa}

A distribuição binomial negativa tem sido muito utilizada como uma primeira alternativa quando a distribuição de Poisson mostra-se inadequada. Muitas vezes os dados apresentam uma grande variabilidade comparada com aquela predita pela variância em modelos de Poisson. Uma causa provável desse fenômeno, denominado sobredispersão, é a heterogeneidade das unidades amostrais que pode ser causada por variabilidades interunidades experimentais. Ao não se considerar a sobredispersão pode-se produzir subestimação dos erros padrão, assim como estimação incorreta dos parâmetros da regressão.

\subsection{Distribuição binomial negativa}

A distribuição binomial negativa pode ser construída como sendo uma distribuição de duas etapas para a resposta de contagem $Y$, supondo $Y \mid Z=z \sim P(z)$ com função de probabilidades denotada por $f(y \mid z)$ e com $Z \sim \operatorname{Gama}(\mu, \phi)$, sendo $\mu>0, \phi>0$, em que $\phi$ não depende de $\mu$. Neste caso obtemos $\mathrm{E}(Z)=\mu$ e $\operatorname{Var}(Z)=\frac{\mu^{2}}{\phi}$. Temos então que

$$
f(y \mid z)=\frac{e^{-z} z^{y}}{y !} \text { e } g(z ; \mu, \phi)=\frac{1}{\Gamma(\phi)}\left(\frac{z \phi}{\mu}\right)^{\phi} e^{-\frac{\phi z}{\mu}} \frac{1}{z} .
$$

Então segue que

$$
\begin{aligned}
\mathrm{E}(Y) & =\mathrm{E}[\mathrm{E}(Y \mid Z)]=\mu \quad \text { e } \\
\operatorname{Var}(Y) & =\mathrm{E}[\operatorname{Var}(Y \mid Z)]+\operatorname{Var}[\mathrm{E}(Y \mid Z)] \\
& =\mu+\mu^{2} / \phi .
\end{aligned}
$$


A função de probabilidades de $Y$ é obtida da seguinte forma:

$$
\begin{aligned}
\operatorname{Pr}\{Y=y\} & =\int_{0}^{\infty} f(y \mid z) g(z ; \mu, \phi) d z \\
& =\frac{1}{y ! \phi}\left(\frac{\phi}{\mu}\right)^{\phi} \int_{0}^{\infty} e^{-z(1+\phi / \mu)} z^{\phi+y-1} d z .
\end{aligned}
$$

Fazendo a transformação de variável $t=z\left(1+\frac{\phi}{\mu}\right)$ temos que $\frac{d z}{d t}=\left(1+\frac{\phi}{\mu}\right)^{-1}$. Segue então

$$
\begin{aligned}
\operatorname{Pr}\{Y=y\} & =\frac{1}{y ! \Gamma(\phi)}\left(\frac{\phi}{\mu}\right)^{\phi}\left(1+\frac{\phi}{\mu}\right)^{-(\phi+y)} \int_{0}^{\infty} e^{-t} t^{\phi+y-1} d t \\
& =\frac{\Gamma(\phi+y) \mu^{y} \phi^{\phi}}{\Gamma(\phi) \Gamma(y+1)(\mu+\phi)^{\phi+y}} \\
& =\frac{\Gamma(\phi+y)}{\Gamma(\phi) \Gamma(y+1)}\left(\frac{\mu}{\mu+\phi}\right)^{y}\left(\frac{\phi}{\mu+\phi}\right)^{\phi} \\
& =\frac{\Gamma(\phi+y)}{\Gamma(\phi) \Gamma(y+1)}(1-\pi)^{\phi} \pi^{y}, \quad y=0,1,2, \ldots,
\end{aligned}
$$

$\operatorname{com} \pi=\mu /(\mu+\phi)$. Portanto, $Y$ segue distribuição binomial negativa de média $\mu$ e parâmetro de dispersão $\phi$. Em consequência, a distribuição binomial negativa possui a mesma média que a distribuição de Poisson, porém apresenta uma variância maior que a média. Se a variável aleatória $Y$ segue $(2.1)$ denotaremos $Y \sim \operatorname{BN}(\mu, \phi)$. Quando $\phi$ é conhecido a distribuição binomial negativa pertence à família exponencial.

\subsection{Modelos com resposta binomial negativa}

Suponha que $Y_{1}, \ldots, Y_{n}$ são variáveis aleatórias independentes tais que $Y_{i} \sim \operatorname{BN}\left(\mu_{i}, \phi\right)$. A função de probabilidades de $Y_{i}$ fica dada por

$$
f\left(y_{i} ; \mu_{i}, \phi\right)=\frac{\Gamma\left(\phi+y_{i}\right)}{\Gamma\left(y_{i}+1\right) \Gamma(\phi)}\left(\frac{\mu_{i}}{\mu_{i}+\phi}\right)^{y_{i}}\left(\frac{\phi}{\mu_{i}+\phi}\right)^{\phi}, \quad y_{i}=0,1,2, \ldots
$$

Temos que $\mathrm{E}\left(Y_{i}\right)=\mu_{i}$ e $\operatorname{Var}\left(Y_{i}\right)=\mu_{i}+\mu_{i}^{2} / \phi$. Similarmente aos MLGs assumimos parte sistemática dada por $g\left(\mu_{i}\right)=\eta_{i}=\mathbf{x}_{i}^{T} \boldsymbol{\beta}$, em que $\mathbf{x}_{i}=\left(x_{i 1}, \ldots, x_{i p}\right)^{T}$ representa os valores das variáveis explicativas, $\boldsymbol{\beta}=\left(\beta_{1}, \ldots, \beta_{p}\right)^{T}, p<n$, é um vetor de parâmetros desconhecidos e $g(\cdot)$ é uma função monótona e diferenciável, denominada função de ligação. Definindo $\boldsymbol{\theta}=\left(\boldsymbol{\beta}^{T}, \phi\right)^{T}$ o logaritmo da função de verossimilhança fica dado por

$$
\mathrm{L}(\boldsymbol{\theta})=\sum_{i=1}^{n}\left[\log \left\{\frac{\Gamma\left(\phi+y_{i}\right)}{\Gamma\left(y_{i}+1\right) \Gamma(\phi)}\right\}+\phi \log \phi+y_{i} \log \mu_{i}-\left(\phi+y_{i}\right) \log \left(\mu_{i}+\phi\right)\right]
$$

em que $\mu_{i}=g^{-1}\left(\mathbf{x}_{i}^{T} \boldsymbol{\beta}\right)$. A fim de obtermos a função escore para $\boldsymbol{\beta}$ calculamos inicialmente 
as derivadas

$$
\begin{aligned}
\frac{\partial \mathrm{L}(\boldsymbol{\theta})}{\partial \beta_{j}} & =\sum_{i=1}^{n}\left\{\frac{y_{i}}{\mu_{i}} \frac{d \mu_{i}}{d \eta_{i}} \frac{\partial \eta_{i}}{\partial \beta_{j}}-\frac{\left(\phi+y_{i}\right)}{\left(\phi+\mu_{i}\right)} \frac{d \mu_{i}}{d \eta_{i}} \frac{\partial \eta_{i}}{\partial \beta_{j}}\right\} \\
& =\sum_{i=1}^{n}\left\{\frac{y_{i}}{\mu_{i}} \frac{d \mu_{i}}{d \eta_{i}} x_{i j}-\frac{\left(\phi+y_{i}\right)}{\left(\phi+\mu_{i}\right)} \frac{d \mu_{i}}{d \eta_{i}} x_{i j}\right\} \\
& =\sum_{i=1}^{n}\left\{\frac{\phi\left(d \mu_{i} / d \eta_{i}\right)}{\mu_{i}\left(\phi+\mu_{i}\right)}\left(y_{i}-\mu_{i}\right) x_{i j}\right\} \\
& =\sum_{i=1}^{n} w_{i} f_{i}^{*-1}\left(y_{i}-\mu_{i}\right) x_{i j},
\end{aligned}
$$

em que $w_{i}=\left(d \mu_{i} / d \eta_{i}\right)^{2} /\left(\mu_{i}^{2} \phi^{-1}+\mu_{i}\right)$ e $f_{i}^{*}=d \mu_{i} / d \eta_{i}$.

Tabela 2.1: Quantidades $w_{i}$ e $f_{i}$ para algumas ligações utilizadas em modelos com resposta binomial negativa.

\begin{tabular}{c|c|c}
\hline Ligação & $w_{i}$ & $f_{i}^{*}$ \\
\hline $\log \mu_{i}=\eta_{i}$ & $\mu_{i}\left(\mu_{i} \phi_{-1}+1\right)^{-1}$ & $\mu_{i}$ \\
$\sqrt{\mu_{i}}=\eta_{i}$ & $4\left(\mu_{i} \phi_{-1}+1\right)^{-1}$ & $2 \sqrt{\mu_{i}}$ \\
$\mu_{i}=\eta_{i}$ & $\left(\mu_{i}^{2} \phi_{-1}+\mu_{i}\right)^{-1}$ & 1 \\
\hline
\end{tabular}

A Tabela 2.1 apresenta as expressões para $w_{i}$ e $f_{i}^{*}$ para algumas ligações que são mais utilizadas em modelos com resposta binomial negativa.

Podemos expressar a função escore para $\boldsymbol{\beta}$ na forma matricial

$$
\mathbf{U}_{\beta}(\boldsymbol{\theta})=\mathbf{X}^{T} \mathbf{W} \mathbf{F}^{-1}(\mathbf{y}-\boldsymbol{\mu})
$$

em que $\mathbf{X}$ é a matrix modelo com linhas $\mathbf{x}_{i}^{T}, i=1, \ldots, n, \mathbf{W}=\operatorname{diag}\left\{w_{1}, \ldots, w_{n}\right\}, \mathbf{F}=$ $\operatorname{diag}\left\{f_{1}^{*}, \ldots, f_{n}^{*}\right\}, \mathbf{y}=\left(y_{1}, \ldots, y_{n}\right)^{T}$ e $\boldsymbol{\mu}=\left(\mu_{1}, \ldots, \mu_{n}\right)^{T}$. Analogamente, a função escore para $\phi$ fica dada por

$$
\mathrm{U}_{\phi}(\boldsymbol{\theta})=\sum_{i=1}^{n}\left[\psi\left(\phi+y_{i}\right)-\psi(\phi)-\frac{\left(y_{i}+\phi\right)}{\left(\phi+\mu_{i}\right)}+\log \left\{\frac{\phi}{\left(\phi+\mu_{i}\right)}\right\}+1\right]
$$

em que $\psi(\cdot)$ é a função digama. Para obtermos a matriz de informação de Fisher de $\boldsymbol{\beta}$ calculamos as derivadas

$$
\begin{aligned}
\frac{\partial^{2} \mathrm{~L}(\boldsymbol{\theta})}{\partial \beta_{j} \partial \beta_{l}}= & -\sum_{i=1}^{n}\left\{\frac{\left(\phi+y_{i}\right)}{\left(\phi+\mu_{i}\right)^{2}}-\frac{y_{i}}{\mu_{i}^{2}}\right\}\left(\frac{d \mu_{i}}{d \eta_{i}}\right)^{2} x_{i j} x_{i l}+ \\
& +\sum_{i=1}^{n}\left\{\frac{y_{i}}{\mu_{i}}-\frac{\left(\phi+y_{i}\right)}{\left(\phi+\mu_{i}\right)}\right\} \frac{d^{2} \mu_{i}}{d \eta_{i}^{2}} x_{i j} x_{i l},
\end{aligned}
$$


cujos valores esperados ficam dados por

$$
\begin{aligned}
\mathrm{E}\left\{\frac{\partial^{2} \mathrm{~L}(\boldsymbol{\theta})}{\partial \beta_{j} \partial \beta_{l}}\right\} & =-\sum_{i=1}^{n} \frac{\phi\left(d \mu_{i} / d \eta_{i}\right)^{2}}{\left(\phi+\mu_{i}\right)} x_{i j} x_{i l} \\
& =-\sum_{i=1}^{n} w_{i} x_{i j} x_{i l} .
\end{aligned}
$$

Logo, podemos expressar a informação de Fisher para $\boldsymbol{\beta}$ em forma matricial

$$
\mathbf{K}_{\beta \beta}(\boldsymbol{\theta})=\mathrm{E}\left\{-\frac{\partial^{2} \mathrm{~L}(\boldsymbol{\theta})}{\partial \boldsymbol{\beta} \partial \boldsymbol{\beta}^{T}}\right\}=\mathbf{X}^{T} \mathbf{W X}
$$

Lawless (1987) mostra que a informação de Fisher para $\phi$ pode ser expressa na forma

$$
\mathbf{K}_{\phi \phi}(\boldsymbol{\theta})=\sum_{i=1}^{n}\left\{\sum_{j=1}^{\infty}(\phi+j)^{-2} \operatorname{Pr}\left(Y_{i} \geq j\right)-\frac{\mu_{i}}{\phi\left(\mu_{i}+\phi\right)}\right\}
$$

e que $\boldsymbol{\beta}$ e $\phi$ são parâmetros ortogonais. Assim, a matriz de informação de Fisher para $\boldsymbol{\theta}$ assume a forma bloco diagonal

$$
\mathbf{K}_{\theta \theta}=\left[\begin{array}{cc}
\mathbf{K}_{\beta \beta} & \mathbf{0} \\
\mathbf{0} & \mathbf{K}_{\phi \phi}
\end{array}\right]
$$

As estimativas de máxima verossimilhança para $\boldsymbol{\beta}$ e $\phi$ podem ser obtidas através de um algoritmo de mínimos quadrados reponderados, aplicando o método escore de Fisher, a partir de (2.2) e do método de Newton-Raphson para obter $\hat{\phi}$ desenvolvido a partir de (2.3), os quais são descritos abaixo

$$
\boldsymbol{\beta}^{(m+1)}=\left(\mathbf{X}^{T} \mathbf{W}^{(m)} \mathbf{X}\right)^{-1} \mathbf{X}^{T} \mathbf{W}^{(m)} \mathbf{y}^{*(m)}
$$

e

$$
\phi^{(m+1)}=\phi^{(m)}-\frac{U_{\phi}^{(m)}}{\ddot{L}_{\phi \phi}^{(m)}},
$$

para $m=1,2, \ldots$, em que

$$
\mathbf{y}^{*}=\mathbf{X} \boldsymbol{\beta}+\mathbf{F}^{-1}(\mathbf{y}-\boldsymbol{\mu})
$$

é uma variável dependente modificada e

$$
\ddot{\mathrm{L}}_{\phi \phi}=\frac{\partial^{2} \mathrm{~L}(\boldsymbol{\theta})}{\partial \phi^{2}}=\sum_{i=1}^{n}\left\{\psi^{\prime}\left(\phi+y_{i}\right)+\frac{\left(y_{i}-2 \mu_{i}-\phi\right)}{\left(\phi+\mu_{i}\right)^{2}}\right\}+\frac{n}{\phi}\left[1-\phi \psi^{\prime}(\phi)\right] .
$$

Os dois procedimentos são aplicados simultaneamente até a convergência. Podemos mostrar ainda que, assintoticamente, ou seja, para $n$ grande, $\hat{\boldsymbol{\beta}}$ e $\hat{\phi}$ são independentes e seguem distribuição aproximadamente normal, ou seja, $\hat{\boldsymbol{\beta}} \sim \mathrm{N}_{p}\left(\boldsymbol{\beta}, \mathbf{K}_{\beta \beta}^{-1}\right)$ e $\hat{\phi} \sim \mathrm{N}\left(\phi, \mathrm{K}_{\phi \phi}^{-1}\right)$. 


\subsection{Qualidade do ajuste}

A qualidade do ajuste de um MLG é avaliada (quando $\phi$ é conhecido) através da função desvio

$$
\mathrm{D}^{*}(\mathbf{y} ; \hat{\boldsymbol{\mu}})=\phi \mathrm{D}(\mathbf{y} ; \hat{\boldsymbol{\mu}})=2\{\mathrm{~L}(\mathbf{y} ; \mathbf{y})-\mathrm{L}(\hat{\boldsymbol{\mu}} ; \mathbf{y})\}
$$

em que, $\mathrm{L}(\boldsymbol{\mu} ; \mathbf{y})=\sum_{i=1}^{n} \mathrm{~L}\left(\mu_{i}, y_{i}\right) \operatorname{com} \mu_{i}=g^{-1}\left(\eta_{i}\right)$ e $\eta_{i}=\mathbf{x}_{i}^{T} \boldsymbol{\beta}$ e para o modelo saturado $(p=$ $n), \mathrm{L}(\mathbf{y} ; \mathbf{y})=\sum_{i=1}^{n} \mathrm{~L}\left(y_{i}, y_{i}\right)$. A equação (2.4) é uma distância entre o logaritmo da função de verossimilhança do modelo saturado (com $n$ parâmetros) e do modelo sob investigação (com $p$ parâmetros) avaliado na estimativa de máxima verossimilhança $\hat{\boldsymbol{\beta}}$. Um valor pequeno para a função desvio indica que, para um número menor de parâmetros, obtemos um ajuste tão bom quanto o ajuste com o modelo saturado.

Assim, supondo $y_{i}>0 \forall i$, a função desvio para para o modelo binomial negativa, assumindo $\phi$ fixo, fica dada por

$$
\mathrm{D}^{*}(\mathbf{y} ; \hat{\boldsymbol{\mu}})=2 \sum_{i=1}^{n}\left[\phi \log \left\{\frac{\hat{\mu}_{i}+\phi}{y_{i}+\phi}\right\}+y_{i} \log \left\{\frac{y_{i}\left(\hat{\mu}_{i}+\phi\right)}{\hat{\mu}_{i}\left(y_{i}+\phi\right)}\right\}\right],
$$

em que $\hat{\mu}_{i}=g^{-1}\left(\mathbf{x}_{i}^{T} \boldsymbol{\beta}\right)$. Quando $y_{i}=0$ o $i$-ésimo componente da função desvio $\mathrm{D}^{*}(\mathbf{y} ; \hat{\boldsymbol{\mu}})$ fica dado por

$$
\begin{aligned}
\mathrm{d}^{* 2}\left(y_{i} ; \hat{\mu}_{i}\right) & =2\left\{\log f\left(0 ; y_{i}, \phi\right)-\log f\left(0 ; \hat{\mu}_{i}, \phi\right)\right\} \\
& =-2 \phi \log \left\{\frac{\phi}{\hat{\mu}_{i}+\phi}\right\} \\
& =2 \phi \log \left\{\frac{\hat{\mu}_{i}+\phi}{\phi}\right\} .
\end{aligned}
$$

Portanto, os componentes do desvio no caso binomial negativo assumem as seguintes formas:

$$
\mathrm{d}^{* 2}\left(y_{i} ; \hat{\mu}_{i}\right)= \begin{cases}2\left[\phi \log \left\{\frac{\hat{\mu}_{i}+\phi}{y_{i}+\phi}\right\}+y_{i} \log \left\{\frac{y_{i}\left(\hat{\mu}_{i}+\phi\right)}{\hat{\mu}_{i}\left(y_{i}+\phi\right)}\right\}\right] & \text { se } y_{i}>0 \\ 2 \phi \log \left\{\frac{\hat{\mu}_{i}+\phi}{\phi}\right\} & \text { se } y_{i}=0 .\end{cases}
$$

Sob a hipótese de que o modelo adotado está correto, $D^{*}(\mathbf{y} ; \hat{\boldsymbol{\mu}})$ segue para $\phi$ grande e $\mu_{i}$ grande, $\forall i$, uma distribuição qui-quadrado com $(n-p)$ graus de liberdade. Suponha agora que temos a partição $\boldsymbol{\beta}=\left(\boldsymbol{\beta}_{1}^{T}, \boldsymbol{\beta}_{2}^{T}\right)^{T}$ em que $\boldsymbol{\beta}_{1}$ é um vetor $q$-dimensional enquanto $\boldsymbol{\beta}_{2}$ tem dimensão $p-q$ e que $\phi$ é fixo ou conhecido. O teste da razão de verossimilhanças para testarmos $\mathrm{H}_{0}: \boldsymbol{\beta}_{1}=0$ contra $\mathrm{H}_{1}: \boldsymbol{\beta}_{1} \neq 0$ reduz, neste caso, à diferença entre dois desvios

$$
\xi_{R V}=\mathrm{D}^{*}\left(\mathbf{y} ; \hat{\boldsymbol{\mu}}^{0}\right)-\mathrm{D}^{*}(\mathbf{y} ; \hat{\boldsymbol{\mu}})
$$

em que $\hat{\boldsymbol{\mu}}^{0}$ e $\hat{\boldsymbol{\mu}}$ são estimativas de $\boldsymbol{\mu}$ sob $\mathrm{H}_{0}$ e $\mathrm{H}_{1}$, respectivamente. Para $\phi$ desconhecido o 
teste da razão de verossimilhanças fica expresso como

$$
\begin{aligned}
\xi_{R V}= & 2 \sum_{i=1}^{n}\left[\log \left\{\frac{\Gamma\left(\hat{\phi}+y_{i}\right) \Gamma\left(\hat{\phi}^{0}\right)}{\Gamma\left(\hat{\phi}^{0}+y_{i}\right) \Gamma(\hat{\phi})}\right\}+\hat{\phi} \log \left\{\frac{\hat{\phi}}{\hat{\phi}+\hat{\mu}_{i}}\right\}-\right. \\
& \left.-\hat{\phi}^{0} \log \left\{\frac{\hat{\phi}^{0}}{\hat{\phi}^{0}+\hat{\mu}_{i}^{0}}\right\}+y_{i} \log \left\{\frac{\hat{\mu}_{i}\left(\hat{\phi}^{0}+\hat{\mu}_{i}^{0}\right)}{\hat{\mu}_{i}^{0}\left(\hat{\phi}+\hat{\mu}_{i}\right)}\right\}\right],
\end{aligned}
$$

em que $\hat{\phi}^{0}$ e $\hat{\phi}$ são as estimativas de máxima verossimilhança de $\phi$ sob $\mathrm{H}_{0}$ e $\mathrm{H}_{1}$, respectivamente. Para $n$ grande e sob $\mathrm{H}_{0}$ temos que $\xi_{R V} \sim \chi_{q}^{2}$.

\subsection{Técnicas de diagnóstico}

Basicamente em técnicas de diagnóstico são aplicadas medidas de alavanca e medidas de influência para avaliar a sensibilidade das estimativas dos parâmetros. Já para avaliar a adequação do ajuste, bem como possíveis afastamentos da distribuição assumida para os erros, são utilizadas análises de resíduos. O conceito de alavanca (definido com $\partial \hat{y}_{i} / \partial y_{i}$ ) supõe que a variável resposta é contínua, não sendo portanto aplicado diretamente em modelos para dados com resposta discreta. Contudo, é possível em muitos casos derivar medidas de alavanca para esses modelos fazendo analogia com modelos lineares equivalentes. Em particular, para modelos com resposta binomial negativa, temos que na convergência o processo iterativo para obter $\hat{\boldsymbol{\beta}}$ pode ser expresso na forma

$$
\hat{\boldsymbol{\beta}}=\left(\mathbf{X}^{T} \widehat{\mathbf{W}} \mathbf{X}\right)^{-1} \mathbf{X}^{T} \widehat{\mathbf{W}} \hat{\mathbf{y}}^{*}
$$

que é equivalente à solução de mínimos quadrados da regressão linear de $\widehat{\mathbf{W}}^{1 / 2} \hat{\mathbf{y}}^{*}$ contra as colunas de $\widehat{\mathbf{W}}^{1 / 2} \mathbf{X}$. A matriz de projeção (ou de alavancas) para esse modelo fica dada por

$$
\widehat{\mathbf{H}}=\widehat{\mathbf{W}}^{1 / 2} \mathbf{X}\left(\mathbf{X}^{T} \widehat{\mathbf{W}} \mathbf{X}\right)^{-1} \mathbf{X}^{T} \widehat{\mathbf{W}}^{1 / 2}
$$

sendo que o $i$-ésimo elemento da diagonal principal de $\mathbf{H}$ assume a forma

$$
\mathrm{h}_{i i}=\frac{\left(d \mu_{i} / d \eta_{i}\right)^{2}}{\left(\mu_{i} \phi^{-1}+\mu_{i}\right)} \mathbf{x}_{i}^{T}\left(\mathbf{X}^{T} \mathbf{W} \mathbf{X}\right)^{-1} \mathbf{x}_{i}
$$

O gráfico de índices de $\hat{\mathrm{h}}_{i i}$ é sugerido para avaliar possíveis pontos de alavanca em modelos de regressão com resposta binomial negativa.

Estudos de Monte Carlo desenvolvidos por Svetliza (2002) e Svetliza e Paula (2003) indicam boa concordância entre o resíduo componente do desvio padronizado

$$
\mathrm{t}_{D_{i}}=\frac{d^{*}\left(y_{i} ; \hat{\mu}_{i}\right)}{\sqrt{1-\hat{h}_{i i}}}
$$


com a distribuição normal padrão, em que

$$
\mathrm{d}^{*}\left(y_{i} ; \hat{\mu}_{i}\right)= \begin{cases} \pm \sqrt{2}\left[\phi \log \left\{\frac{\hat{\mu}_{i}+\phi}{y_{i}+\phi}\right\}+y_{i} \log \left\{\frac{y_{i}\left(\hat{\mu}_{i}+\phi\right)}{\hat{\mu}_{i}\left(y_{i}+\phi\right)}\right\}\right]^{1 / 2} & \text { se } y_{i}>0 \\ \pm \sqrt{2} \phi \log \left\{\frac{\hat{\mu}_{i}+\phi}{\phi}\right\} & \text { se } y_{i}=0\end{cases}
$$

$\mathrm{O}$ resíduo $\mathrm{t}_{D_{i}}$ pode ser utilizado para detectar observações aberrantes bem como afastamentos da suposição da distribuição binomial negativa para a resposta através do gráfico normal de probabilidades.

Uma versão da distância de Cook (1977) aproximada desenvolvida por Svetliza (2002) fica dada por

$$
\mathrm{LD}_{i}=\frac{\hat{h}_{i i}}{\left(1-\hat{h}_{i i}\right)^{2}} \hat{r}_{P_{i}}^{2}
$$

em que $r_{P_{i}}=\left(y_{i}-\mu_{i}\right) / \sqrt{\operatorname{Var}\left(Y_{i}\right)}$ e $\operatorname{Var}\left(Y_{i}\right)=\mu_{i}+\mu_{i}^{2} / \phi$.

O gráfico de $\mathrm{LD}_{i}$ contra o índice das observações ou valores ajustados pode revelar pontos influentes nas estimativas de $\hat{\boldsymbol{\beta}}$ e $\hat{\phi}$. Svetliza (2002) derivou as curvaturas de influência local em modelos paramétricos com resposta binomial negativa sob esquemas usuais de perturbação.

\subsection{Seleção de modelos}

Procedimentos usuais de seleção de modelos são baseados em medidas de informação, tais como o procedimento de Akaike (1974) que consiste em relacionar o modelo cujo AIC seja mínimo, em que

$$
\mathrm{AIC}=-2 \mathrm{~L}(\hat{\boldsymbol{\theta}})+2 p,
$$

ou o procedimento desenvolvido por Schwarz (1978) que utiliza uma penalização diferente e consiste em minimizar

$$
\mathrm{BIC}=-2 \mathrm{~L}(\hat{\boldsymbol{\theta}})+p \log (n),
$$

com $\mathrm{L}(\hat{\boldsymbol{\theta}})$ sendo o logaritmo da função de verossimilhança avaliado em $\hat{\boldsymbol{\theta}}$. 


\section{Capítulo 3}

\section{Extensão para o caso semiparamétrico}

O modelo semiparamétrico é uma extensão natural do modelo com resposta binomial negativa descrito no Capítulo 2. São adotadas funções não paramétricas para estudar a relação entre a variável resposta e as variáveis explicativas permitindo assim uma maior flexibilidade e não impondo uma forma rígida de dependência na modelagem das variáveis em questão.

Dentre os principais trabalhos relacionados aos modelos semiparamétricos para dados de contagem estão Green e Silverman (1994), em que mostram um estudo detalhado dos modelos semiparamétricos no caso do modelo normal e para os Modelos Lineares Generalizados (MLGs), Rigby e Stasinopoulos (2005) desenvolvem os GAMLSS (Generalized Additive Models for Location Scale and Shape), em que flexibilizam algumas suposições dos MLGs para os modelos aditivos generalizados com a possibilidade de modelar parâmetros de assimetria e curtose, além dos parâmetros de posição e dispersão. Desenvolvem também o pacote gamlss no R, o qual é usado para a aplicação neste trabalho. Li (2010) também estuda a parte inferencial do modelo semiparamétrico com resposta binomial negativa (MSRBN).

Como um dos objetivos deste trabalho é desenvolver métodos de diagnóstico para os modelos semiparamétricos com resposta binomial negativa, introduzimos neste capítulo conceitos de especificação das variáveis, de ajuste via splines cúbicos e do parâmetro de suavização, além de definir as funções de verossimilhança penalizadas e graus de liberdade efetivos. Integram ainda este capítulo a estimação dos parâmetros através da verossimilhança penalizada pelo método iterativo backfitting, estudo dos graus de liberdade efetivos, estimação dos parâmetros de dispersão e suavização.

\subsection{Especificação do modelo}

Suponha que $Y_{i}$, com $i=1, \ldots, n$, são variáveis aleatórias independentes tais que $Y_{i} \sim$ $\mathrm{BN}\left(\mu_{i}, \phi\right)$, em que $\mathrm{E}\left(Y_{i}\right)=\mu_{i}$ e $\operatorname{Var}\left(Y_{i}\right)=\mu_{i}+\mu_{i}^{2} / \phi$ e parte sistemática dada por

$$
g\left(\mu_{i}\right)=\eta_{i}=\mathbf{x}_{i}^{T} \boldsymbol{\beta}+f\left(t_{i}\right),
$$


em que $g(\cdot)$ é uma função de ligação, $\mathbf{x}_{i}=\left(x_{i 1}, \ldots, x_{i p}\right)^{T}$ contém valores de variáveis explicativas, $\boldsymbol{\beta}=\left(\beta_{1}, \ldots, \beta_{p}\right)^{T}$ é um vetor de parâmetros desconhecidos, $f(\cdot)$ é uma função univariada arbitrária não especificada do efeito não paramétrico e $t_{i}$ é o valor da variável explicativa que deve ser controlada de forma não paramétrica.

Alternativamente, o componente sistemático (3.1) pode ser reexpresso na forma

$$
g\left(\mu_{i}\right)=\mathbf{x}_{i}^{T} \boldsymbol{\beta}+\mathbf{n}_{i}^{T} \mathbf{f},
$$

ou ainda na forma matricial

$$
\mathrm{g}(\boldsymbol{\mu})=\mathbf{X} \boldsymbol{\beta}+\mathbf{N f}
$$

em que $\mathbf{n}_{i}$ é um vetor de zeros e uns que dependem de $t_{1}^{0}, \ldots, t_{h}^{0}$ que por sua vez são os valores ordenados e distintos da variável explicativa $t_{i}, \mathbf{f}=\left(f\left(t_{1}^{0}\right), \ldots, f\left(t_{h}^{0}\right)\right)^{T}$ e $\mathbf{N}$ é chamada matriz de incidência $(n \times h)$, com elementos dados pela função indicadora $I\left(t_{i}=t_{j}^{0}\right)$, com $\mathbf{N}=\mathbf{I}_{n}$ quando não há empates, ou seja, quanto todos os valores de $t$ forem distintos.

No modelo semiparamétrico assumimos que $f(\cdot)$ está em um espaço de funções sob determinadas restrições, em que se busca uma melhor combinação linear de funções desse espaço para o ajuste da variável de interesse. Dentre as várias opções de escolha de funções existentes será dado ênfase à estimação com splines cúbicos.

\subsection{Método de suavização spline cúbico}

Os modelos de regressão linear são utilizados em casos em que a relação entre a variável resposta e as variáveis explicativas são conhecidas, neste caso lineares, caso contrário em que conhecemos a relação, não linear, utilizamos os modelos não lineares. No entanto, é comum observarmos relações entre variável resposta e variável explicativa que são não lineares e desconhecidas. Daí surgiram os modelos não paramétricos em que não há a necessidade de prefixar uma relação a priori para ser utilizada no modelo.

Existem diversas técnicas de regressão não paramétrica. Neste trabalho abordamos a técnica apresentada em Montgomery et al. (2001, Capítulo 7), que é a regressão não paramétrica através de splines, em que funções são construídas por meio da junção de várias funções polinomiais de ordem $k$ que foram particionadas da variável explicativa a ser controlada de forma não paramétrica.

Os pontos de junção de cada parte da função polinomial são denominados nós. Vale destacar que o spline é constuído por um conjunto de funções polinomiais contínuas, logo também será contínuo. Os splines cúbicos $(k=3)$ são habitualmente adequados para a maioria dos problema práticos.

Um spline cúbico com $h$ nós, $t_{1}<t_{2}<\ldots<t_{h}$, com a primeira e segunda derivadas 
contínuas, pode ser escrito como

$$
S(x)=\sum_{j=0}^{3} \beta_{0 j} x^{j}+\sum_{i=1}^{h} \beta_{i}\left(x-t_{i}\right)_{+}^{3},
$$

em que

$$
\left(x-t_{i}\right)_{+}= \begin{cases}\left(x-t_{i}\right) & \text { if } x-t_{i}>0 \\ 0 & \text { if } x-t_{i} \leq 0 .\end{cases}
$$

Assume-se neste tipo de modelagem que as posições dos nós são conhecidos, assim o ajuste do spline pode ser feito por mínimos quadrados de forma direta.

A decisão de escolher o número de nós, a posição em que ficarão e o grau do polinômio em cada segmento da função spline não é uma tarefa fácil. A mais complicada é a escolha do número de nós na estimação, uma vez que o ajuste da função spline é muito sensível a essa escolha.

O modelo de spline cúbico básico (3.2) pode ser modificado para ajustar funções polinomiais de diferentes ordens em cada segmento para impor diferentes restrições de continuidade nos nós. Se todos as $h+1$ partes polinomiais são de ordem três, então o modelo spline cúbico com restrições de continuidade é dado por

$$
S(x)=\sum_{j=0}^{3} \beta_{0 j} x^{j}+\sum_{i=1}^{h} \sum_{j=0}^{3} \beta_{i j}\left(x-t_{i}\right)_{+}^{j},
$$

em que $(x-t)_{+}^{0}$ é igual a 1 se $x>t$ e 0 se $x \leq t$. Assim, se o termo $\beta_{i j}\left(x-t_{i}\right)_{+}^{j}$ está no modelo, isso força uma descontinuidade em $t_{i}$ na $j$-ésima derivada de $S(x)$. Se o termo estiver ausente, a j-ésima derivada de $S(x)$ é contínua em $t_{i}$. Quanto menor forem as restrições de continuidade melhor será para o ajuste da função, pois mais parâmetros estão no modelo e vice e versa. A determinação da ordem dos segmentos polinomiais e das restrições de continuidade, sem prejudicar o ajuste, pode ser feita através de testes de hipóteses da regressão linear usual.

Como ilustração, considere um spline cúbico com um nó em $t$ e restrições de continuidade

$$
\begin{aligned}
S(x) & =\beta_{00}+\beta_{01} x+\beta_{02} x^{2}+\beta_{03} x^{3}+\beta_{10}(x-t)_{+}^{0} \\
& +\beta_{11}(x-t)_{+}^{1}+\beta_{12}(x-t)_{+}^{2}+\beta_{13}(x-t)_{+}^{3} .
\end{aligned}
$$

Note que $S(x), S^{\prime}(x)$ e $S^{\prime \prime}(x)$ não são necessariamente contínuas em $t$, uma vez que os termos $\beta_{10}, \beta_{11}$ e $\beta_{12}$ estão no modelo. Para determinar qual restrição de continuidade reduz a qualidade do ajuste, os testes para avaliar as hipóteses $H_{0}: \beta_{10}=0$ (continuidade de $S(x)$ ), $H_{0}: \beta_{10}=\beta_{11}=0$ (continuidade de $S(x)$ e $\left.S^{\prime}(x)\right)$ e $H_{0}: \beta_{10}=\beta_{11}=\beta_{12}=0$ (continuidade de $S(x), S^{\prime}(x)$ e $S^{\prime \prime}(x)$ ) podem ser aplicados e para saber se o spline cúbico se ajusta melhor aos dados em relação à função polinomial cúbica em toda amplitude de $x$, basta testar $H_{0}: \beta_{10}=\beta_{11}=\beta_{12}=\beta_{13}=0$. 
Uma desvantagem deste método é que a matriz $\mathbf{X}^{T} \mathbf{X}$ começa a ter problemas de singularidade se for escolhido um número de nós muito grande. Este problema pode ser contornado utilizando uma representação diferente de spline, chamado de B-spline cúbico, que é dada por

$$
B_{i}(x)=\sum_{j=i-4}^{i}\left[\frac{\left(x-t_{j}\right)_{+}^{3}}{\prod_{\substack{m=i-4 \\ m \neq j}}^{i}\left(t_{j}-t_{m}\right)}\right]
$$

$\mathrm{e}$

$$
S(x)=\sum_{i=1}^{h+4} \gamma_{i} B_{i}(x)
$$

para $i=1,2, \ldots, h+4$, em que $\gamma_{i}$ são os parâmetros a serem estimados. Em (3.3) temos oito nós adicionais, $t_{-3}<t_{-2}<t_{-1}<t_{0}$ e $t_{h+1}<t_{h+2}<t_{h+4}<t_{h+4}$. Usualmente tomamos $t_{0}=x_{\min }$ e $t_{h}+1=x_{\max }$ com os outros nós escolhidos arbitrariamente. Mais detalhes podem ser consultados em De Boor (1977) e Dierckx (1993), por exemplo.

Dentre os splines cúbicos, existem splines variantes segundo determinadas restrições. Por exemplo, podemos destacar os splines cúbicos naturais ( $\mathrm{SCN}$ ), que além das propriedades do spline cúbico, possuem uma propriedade adicional que, nos intervalos $\left.]-\infty ; t_{1}\right]$ e $\left[t_{h} ;+\infty[\right.$ a segunda derivada de $f(\cdot)$ é zero, ou seja, $f(\cdot)$ é uma função linear a partir dos nós extremos. Para mais detalhes referentes aos splines cúbicos naturais, consultar De Boor (1978) ou Reinsch (1967).

\subsection{Critério de penalização}

Ao estimarmos os parâmetros dos splines por mínimos quadrados, a escolha do número de nós utilizados é ainda mais importante. Se escolhermos um número insuficiente de nós, a curva estimada pode não estar bem ajustada. Por outro lado, se escolhermos um número muito grande de nós, obteremos um ajuste perto da interpolação dos dados, o que também não é adequado. Como a qualidade do ajuste da função não paramétrica depende muito da escolha do número de nós, usualmente é escolhido um grande número de nós chegando, ou quase, a interpolar os dados e posteriormente para suavizar a função adicionamos um critério de penalização. 


\subsubsection{Função de penalização}

Neste trabalho vamos abordar dois tipos função de penalização. A primeira que veremos foi introduzida por O'Sullivan (1986), em que a penalização é aplicada na curva $f(\cdot)$.

O critério de penalização é baseado na derivada de segunda ordem de $f(\cdot)$ e avaliando sua integral em todo domínio $[a, b]=\left[t_{1}, t_{h}\right]$ de $t$, de forma que quanto maior a curvatura da função, maior o valor da derivada avaliada e, consequentemente, maior o valor da integral. Neste caso a penalização é dada por

$$
\int_{a}^{b}\left[f^{\prime \prime}(t)\right]^{2} d t
$$

De acordo com Green e Silverman (1994) podemos expressar (3.4) para SCN na seguinte forma:

$$
\int_{a}^{b}\left[f^{\prime \prime}(t)\right]^{2} d t=\mathbf{f}^{T} \mathbf{K} \mathbf{f}
$$

em que $\mathbf{f}$ é o vetor $(h \times 1)$ de parâmetros a serem estimados e $\mathbf{K}$ é uma matriz $(h \times h)$ positiva definida que depende dos nós, com estrutura dada por $\mathbf{K}=\mathbf{Q R}^{-1} \mathbf{Q}^{T}$, em que $\mathbf{Q}$ é uma matriz tridiagonal $h \times(h-2)$ e $\mathbf{R}$ é uma matriz simétrica tridiagonal $(h-2) \times(h-2)$.

Para definirmos os elementos das matrizes $\mathbf{Q}$ e $\mathbf{R}$, seja $u_{i}=t_{i+1}^{0}-t_{i}^{0}$, ou seja, a distância entre dois nós consecutivos e distintos $i$ e $i+1$. Os elementos $q_{i j}$ da matriz $\mathbf{Q}$ são dados por

$$
q_{(j-1) j}=u_{j-1}^{-1}, \quad q_{j j}=-u_{j-1}^{-1}-u_{j}^{-1}, \quad \text { e } \quad q_{(j+1) j}=u_{j}^{-1}
$$

e $q_{i j}=0$ para $|i-j|>1, i=1, \ldots, h, j=1, \ldots, h-1$.

Já os elementos $r_{i j}, j=2, \ldots, h-1$, de $\mathbf{R}$ são dados por

$$
\begin{gathered}
r_{i i}=\frac{1}{3}\left(u_{i-1}+u_{i}\right), \quad i=1, \ldots, h-2, \\
r_{i(i+1)}=r_{(i+1) i}=\frac{1}{6} u_{i} \quad i=1, \ldots, h-1
\end{gathered}
$$

e $r_{i j}=0$ para $|i-j|>1$.

Um outro tipo de penalização proposto por Eilers e Marx (1996), que deram o nome de P-Splines, vem alcançando grande popularidade recentemente (Ruppert et al., 2003). A penalização é baseada nas diferenças entre os coeficientes adjacentes e é uma penalização direta dos coeficientes, ao invés de penalizar a curva, reduzindo assim a dimensionalidade do problema.

Eilers e Marx (1996) usam uma penalização baseada em diferenças de ordem $d$ entre coeficientes adjacentes de bases B-splines. Tais penalizações são mais flexíveis uma vez que são independentes do grau do polinômio utilizado para construir os B-splines. Esta é uma boa aproximação discreta para a integral da $d$-ésima derivada ao quadrado. 
A penalização é dada por

$$
\mathbf{f}^{T} \mathbf{P}_{d} \mathbf{f}
$$

em que $\mathbf{P}_{d}=\left(\Delta^{d}\right)^{T}\left(\Delta^{d}\right)$ e $\Delta^{d}$ é o operador diferença de ordem $d$.

Normalmente é utilizado diferenças de ordem $d=2$, ainda que dê para aplicar ordens mais elevadas ou mais baixas dependendo da variabilidade da curva e da quantidade de ruído presente nos dados. Por exemplo, a penalização e ordem $d=2$ é equivalente a

$$
\mathbf{f}^{T}\left(\Delta^{2}\right)^{T} \Delta^{2} \mathbf{f}=\left(f_{1}-2 f_{2}+f_{3}\right)^{2}+\ldots+\left(f_{h-2}-2 f_{h-1}+f_{h}\right)^{2}
$$

em que $\Delta^{2}$ é dada por

$$
\boldsymbol{\Delta}^{\mathbf{2}}=\left[\begin{array}{ccccc}
1 & -2 & 1 & 0 & \ldots \\
0 & 1 & -2 & 1 & \ldots \\
0 & 0 & 1 & -2 & \ldots \\
\vdots & \vdots & \vdots & \vdots & \ddots
\end{array}\right]
$$

\subsubsection{Logaritmo da função de verossimilhança penalizada}

Seja $\boldsymbol{\theta}=\left(\boldsymbol{\beta}^{T}, \mathbf{f}^{T}, \phi\right)^{T}$ o vetor de parâmetros a ser estimado no modelo (3.1), com espaços paramétricos $\Theta_{\boldsymbol{\beta}} \in \mathbb{R}, \Theta_{\mathbf{f}} \in \mathbb{R}$ e $\Theta_{\phi} \in \mathbb{R}^{+}$. A função de verossimilhança no MSRBN é o produto das funções de probabilidades da distribuição binomial negativa, dado por

$$
\mathrm{L}(\boldsymbol{\theta})=\prod_{i=1}^{n} \frac{\Gamma\left(\phi+y_{i}\right)}{\Gamma\left(y_{i}+1\right) \Gamma(\phi)}\left(\frac{\mu_{i}}{\mu_{i}+\phi}\right)^{y_{i}}\left(\frac{\phi}{\mu_{i}+\phi}\right)^{\phi}
$$

em que $g\left(\mu_{i}\right)=\mathbf{x}_{i}^{T} \boldsymbol{\beta}+f\left(t_{i}\right)$. Como mencionado na Seção 3.3, o acréscimo de uma função de penalização é necessário para se obter uma boa estimação para a curva $f(\cdot)$, ou seja, diminuindo o grau de ruído da curva. Seja $\mathrm{L}_{p}(\boldsymbol{\theta}, \lambda)$ o logaritmo da função de verossimilhança penalizada

$$
\begin{aligned}
\mathrm{L}_{p}(\boldsymbol{\theta}, \lambda) & =\sum_{i=1}^{n}\left[\log \left\{\frac{\Gamma\left(\phi+y_{i}\right)}{\Gamma\left(y_{i}+1\right) \Gamma(\phi)}\right\}+\phi \log \phi+y_{i} \log \mu_{i}-\left(\phi+y_{i}\right) \log \left(\mu_{i}+\phi\right)\right]- \\
& -\frac{\lambda}{2} \mathbf{f}^{T} \mathbf{K f}
\end{aligned}
$$

em que $\lambda>0$ é o parâmetro de suavização. Para $\lambda \rightarrow 0$, a segunda parcela de (3.6) tem pouca influência e prioriza o ajuste por interpolação da curva, enquanto para $\lambda \rightarrow \infty$ prioriza a suavidade da curva $f(\cdot)$, sob a restrição $f^{\prime \prime}(\cdot)=0$ para a curva ser linear.

Da mesma forma se utilizarmos a penalização P-splines o logaritmo da função de veros- 
similhança penalizada fica dado por

$$
\begin{aligned}
\mathrm{L}_{p}(\boldsymbol{\theta}, \lambda) & =\sum_{i=1}^{n}\left[\log \left\{\frac{\Gamma\left(\phi+y_{i}\right)}{\Gamma\left(y_{i}+1\right) \Gamma(\phi)}\right\}+\phi \log \phi+y_{i} \log \mu_{i}-\left(\phi+y_{i}\right) \log \left(\mu_{i}+\phi\right)\right]- \\
& -\frac{\lambda}{2} \mathbf{f}^{T} \mathbf{P}_{d} \mathbf{f} .
\end{aligned}
$$

A partir da Seção 3.3.3 iremos introduzir o desenvolvimento de todas as contas considerando a penalização 3.4, entretanto se quisermos utilizar a penalização P-splines dada em 3.5 , basta trocarmos a matriz $\mathbf{K}$ pela matriz $\mathbf{P}_{d}$.

\subsubsection{Função escore e informação de Fisher penalizadas}

Considerando $\lambda$ fixo, seja $\mathbf{U}_{p}(\boldsymbol{\theta})$ o vetor de funções escore penalizadas $\left(p^{\prime} \times 1\right)$, em que $p^{\prime}=p+h+1$, dado por

$$
\mathbf{U}_{p}(\boldsymbol{\theta})=\left(\begin{array}{c}
\mathbf{U}_{p}^{\boldsymbol{\beta}}(\boldsymbol{\theta}) \\
\mathbf{U}_{p}^{\mathbf{f}}(\boldsymbol{\theta}) \\
\mathbf{U}_{p}^{\phi}(\boldsymbol{\theta})
\end{array}\right),
$$

com $\mathbf{U}_{p}^{\boldsymbol{\beta}}(\boldsymbol{\theta})$ sendo um vetor $(p \times 1)$ de funções escore referentes à parte paramétrica, $\mathbf{U}_{p}^{\mathbf{f}}(\boldsymbol{\theta})$ é um vetor $(h \times 1)$ de funções escore referentes à parte não paramétrica e $\mathrm{U}_{p}^{\phi}(\boldsymbol{\theta})$ é a função escore referente ao parâmetro de dispersão. Após algumas manipulações algébricas similares ao Capítulo 2 (ver Apêndice B) obtemos

$$
\begin{aligned}
\mathbf{U}_{p}^{\boldsymbol{\beta}}(\boldsymbol{\theta}) & =\mathbf{X}^{T} \mathbf{W} \mathbf{F}^{-1}(\mathbf{y}-\boldsymbol{\mu}), \\
\mathbf{U}_{p}^{\mathbf{f}}(\boldsymbol{\theta}) & =\mathbf{N}^{T} \mathbf{W} \mathbf{F}^{-1}(\mathbf{y}-\boldsymbol{\mu})-\lambda \mathbf{K f} \mathbf{e} \\
\mathrm{U}_{p}^{\phi}(\boldsymbol{\theta}) & =\sum_{i=1}^{n}\left[\psi\left(\phi+y_{i}\right)-\psi(\phi)-\frac{\left(y_{i}+\phi\right)}{\left(\phi+\mu_{i}\right)}+\log \left\{\frac{\phi}{\left(\phi+\mu_{i}\right)}\right\}+1\right] .
\end{aligned}
$$

Podemos reescrever (3.8) e (3.9) como

$$
\begin{aligned}
\mathbf{U}_{p}^{\boldsymbol{\beta}}(\boldsymbol{\theta}) & =\mathbf{X}^{T} \mathbf{W} \mathbf{z}, \\
\mathbf{U}_{p}^{\mathbf{f}}(\boldsymbol{\theta}) & =\mathbf{N}^{T} \mathbf{W} \mathbf{z}-\lambda \mathbf{K} \mathbf{f},
\end{aligned}
$$

em que $\mathbf{z}$ é uma variável dependente modificada e é dada por $\mathbf{z}=\mathbf{F}^{-1}(\mathbf{y}-\boldsymbol{\mu})$.

Apresentamos a seguir a matriz de informação de Fisher penalizada, obtendo primeiramente a matriz de informação observada de Fisher penalizada, que é definida por

$$
-\ddot{\mathbf{L}}_{p}^{\boldsymbol{\theta} \boldsymbol{\theta}}=\frac{\partial^{2} \mathrm{~L}_{p}(\boldsymbol{\theta}, \lambda)}{\partial \boldsymbol{\theta} \partial \boldsymbol{\theta}^{T}},
$$


em que $\ddot{\mathbf{L}}_{p}^{\boldsymbol{\theta} \boldsymbol{\theta}}$ é a matriz Hessiana penalizada dada por

$$
\ddot{\mathbf{L}}_{p}^{\boldsymbol{\theta} \boldsymbol{\theta}}=\left(\begin{array}{ccc}
\ddot{\mathbf{L}}_{p}^{\boldsymbol{\beta} \boldsymbol{\beta}} & \ddot{\mathbf{L}}_{p}^{\boldsymbol{\beta f}} & \ddot{\mathbf{L}}_{p}^{\boldsymbol{\beta} \phi} \\
\ddot{\mathbf{L}}_{p}^{\mathbf{\beta}} & \ddot{\mathbf{L}}_{p}^{\mathrm{ff}} & \ddot{\mathbf{L}}_{p}^{\mathbf{f} \phi} \\
\ddot{\mathbf{L}}_{p}^{\phi \boldsymbol{\beta}} & \ddot{\mathbf{L}}_{p}^{\phi \mathbf{f}} & \ddot{\mathrm{L}}_{p}^{\phi \phi}
\end{array}\right)
$$

com

$$
\begin{aligned}
\ddot{\mathbf{L}}_{p}^{\boldsymbol{\beta} \mathbf{f}} & =\left(\ddot{\mathbf{L}}_{p}^{\mathbf{f} \boldsymbol{\beta}}\right)^{T}, \\
\ddot{\mathbf{L}}_{p}^{\boldsymbol{\beta} \phi} & =\left(\ddot{\mathbf{L}}_{p}^{\phi \boldsymbol{\beta}}\right)^{T} \mathrm{e} \\
\ddot{\mathbf{L}}_{p}^{\mathbf{f} \phi} & =\left(\ddot{\mathbf{L}}_{p}^{\phi \mathbf{f}}\right)^{T},
\end{aligned}
$$

portanto tem-se simetria da matriz Hessiana.

Após algumas operações algébricas (ver Apêndice B) temos que as submatrizes bloco diagonal da matriz Hessiana penalizada podem ser escritas na forma

$$
\begin{aligned}
\ddot{\mathbf{L}}_{p}^{\boldsymbol{\beta} \boldsymbol{\beta}} & =-\mathbf{X}^{T} \mathbf{W} \mathbf{X}-\mathbf{X}^{T} \mathbf{A}(\mathbf{a}) \mathbf{X}(\mathbf{y}-\boldsymbol{\mu}), \\
\ddot{\mathbf{L}}_{p}^{\mathrm{ff}} & =-\mathbf{N}^{T} \mathbf{W} \mathbf{N}-\mathbf{N}^{T} \mathbf{A}(\mathbf{a}) \mathbf{N}(\mathbf{y}-\boldsymbol{\mu})-\lambda \mathbf{K} \mathrm{e} \\
\ddot{\mathrm{L}}_{p}^{\phi \phi} & =\sum_{i=1}^{n}\left\{\psi^{\prime}\left(\phi+y_{i}\right)+\frac{\left(y_{i}-2 \mu_{i}-\phi\right)}{\left(\phi+\mu_{i}\right)^{2}}\right\}+\frac{n}{\phi}\left[1-\phi \psi^{\prime}(\phi)\right],
\end{aligned}
$$

em que a função $\psi^{\prime}(\cdot)$ é chamada função trigama, $\mathbf{A}(\mathbf{a})=\operatorname{diag}\left\{a_{1}, \ldots, a_{n}\right\}$, com $a_{i}=$ $\left(\frac{d \mu_{i}}{d \eta_{i}}\right)^{2} \frac{2 \phi^{-1} \mu_{i}+1}{\left(\mu_{i}^{2} \phi^{-1}+\mu_{i}\right)^{2}}$, para $i=1, \ldots, n$.

Já as submatrizes cruzadas podem ser expressas na seguinte forma:

$$
\begin{aligned}
\ddot{\mathbf{L}}_{p}^{\boldsymbol{\beta f}} & =-\mathbf{X}^{T} \mathbf{W} \mathbf{N}-\mathbf{X}^{T} \mathbf{A}(\mathbf{a}) \mathbf{N}(\mathbf{y}-\boldsymbol{\mu}) \\
\ddot{\mathbf{L}}_{p}^{\boldsymbol{\beta} \phi} & =\mathbf{X}^{T} \mathbf{A}(\mathbf{b})(\mathbf{y}-\boldsymbol{\mu}) \text { e } \\
\ddot{\mathbf{L}}_{p}^{\mathbf{f} \phi} & =\mathbf{N}^{T} \mathbf{A}(\mathbf{c})(\mathbf{y}-\boldsymbol{\mu})
\end{aligned}
$$

em que $\mathbf{A}(\mathbf{b})=\operatorname{diag}\left\{b_{1}, \ldots, b_{n}\right\}$ e $\mathbf{A}(\mathbf{c})=\operatorname{diag}\left\{c_{1}, \ldots, c_{n}\right\}, \operatorname{com} b_{i}=\left(\frac{d \mu_{i}}{d \eta_{i}}\right) \frac{\phi^{-2}}{\left(\mu_{i} \phi^{-1}+1\right)^{2}} \mathrm{e}$ $c_{i}=\left(\frac{d \mu_{i}}{d \eta_{i}}\right) \frac{1}{\left(\mu_{i}^{2} \phi^{-1}+\mu_{i}\right)}$, para $i=1, \ldots, n$.

A matriz de informação de Fisher penalizada é definida da seguinte forma:

$$
\mathbf{K}_{p}^{\boldsymbol{\theta} \boldsymbol{\theta}}=-\mathrm{E}\left\{\frac{\partial^{2} \mathrm{~L}_{p}(\boldsymbol{\theta}, \lambda)}{\partial \boldsymbol{\theta} \partial \boldsymbol{\theta}^{T}}\right\}
$$


cujas submatrizes são dadas por

$$
\mathbf{K}_{p}^{\boldsymbol{\theta} \boldsymbol{\theta}}=\left(\begin{array}{ccc}
\mathbf{K}_{p}^{\boldsymbol{\beta} \boldsymbol{\beta}} & \mathbf{K}_{p}^{\boldsymbol{\beta f}} & \mathbf{K}_{p}^{\boldsymbol{\beta} \phi} \\
\mathbf{K}_{p}^{\mathbf{f} \boldsymbol{\beta}} & \mathbf{K}_{p}^{\mathrm{ff}} & \mathbf{K}_{p}^{\mathbf{f} \phi} \\
\mathbf{K}_{p}^{\phi \boldsymbol{\beta}} & \mathbf{K}_{p}^{\phi \mathbf{f}} & \mathbf{K}_{p}^{\phi \phi}
\end{array}\right)
$$

Observe que a matriz de informação de Fisher $\mathbf{K}_{\boldsymbol{\theta} \boldsymbol{\theta}}$ é também simétrica.

Após algumas operações algébricas podemos mostrar que as submatrizes bloco diagonal da matriz de informação de Fisher (ver Apêndice B) podem ser escritas na forma

$$
\begin{aligned}
\mathbf{K}_{p}^{\boldsymbol{\beta} \boldsymbol{\beta}} & =\mathbf{X}^{T} \mathbf{W X} \\
\mathbf{K}_{p}^{\mathrm{ff}} & =\mathbf{N}^{T} \mathbf{W} \mathbf{N}+\lambda \mathbf{K} \mathrm{e} \\
\mathrm{K}_{p}^{\phi \phi} & =\sum_{i=1}^{n}\left\{\sum_{j=0}^{\infty} \frac{\mathrm{P}_{r}\left(Y_{i} \geq j\right)}{(\phi+j)^{2}}-\frac{\mu_{i}}{\phi\left(\mu_{i}+\phi\right)}\right\} .
\end{aligned}
$$

Já as submatrizes cruzadas podem ser expressas na seguinte forma:

$$
\begin{aligned}
\mathbf{K}_{p}^{\boldsymbol{\beta} \mathbf{f}} & =\mathbf{X}^{T} \mathbf{W} \mathbf{N} \\
\mathbf{K}_{p}^{\boldsymbol{\beta} \phi} & =\mathbf{0}, \\
\mathbf{K}_{p}^{\mathbf{f} \phi} & =\mathbf{0} .
\end{aligned}
$$

Nota-se ainda que os parâmetros são ortogonais exceto para $\boldsymbol{\beta}$ e $\mathbf{f}$.

A partir dessas matrizes, a manipulação computacional se torna muito mais atrativa, facilitando a estimação dos parâmetros do modelo.

\subsection{Estimação dos parâmetros}

O nosso objetivo é encontrar os valores dos parâmetros do modelo que maximizam sua função de verossimilhança penalizada, ou equivalentemente, os valores que maximizam o logaritmo da função de verossimilhança penalizada.

Há diversas propostas de estimação no contexto de modelos semiparamétricos, porém neste trabalho a estimação dos parâmetros de posição dos MSRBN são realizadas através do algoritmo backfitting.

Supondo que o logaritmo da função de verossimilhança penalizada $\mathrm{L}_{p}(\boldsymbol{\theta}, \lambda)$, definido em (3.6), é uma função duas vezes diferenciável e que satisfaz certas condições de regularidade, o vetor paramétrico $\boldsymbol{\beta}$ e o vetor não paramétrico $\mathbf{f}$ que maximizam $\mathrm{L}_{p}(\boldsymbol{\theta}, \lambda)$ satisfazem a desigualdade

$$
\mathrm{L}_{p}(\hat{\boldsymbol{\theta}}, \lambda) \geq \sup _{\boldsymbol{\theta} \in \Theta} \mathrm{L}_{p}(\boldsymbol{\theta}, \lambda)
$$


Considerando $\lambda$ e $\phi$ fixos utilizamos o algoritmo backfitting para encontrarmos os valores dos parâmetros que maximizam a função acima. De forma geral, o algoritmo backfitting é um método iterativo do escore de Fisher e tenta obter a solução em que as funções escore calculadas na Seção 3.3.3 sejam iguais a 0. É possível mostrar que a $(u+1)$-ésima etapa do processo iterativo escore de Fisher para maximizar a função de verossimilhança penalizada com respeito a $\boldsymbol{\beta}$ e $\mathbf{f}$ para $\phi$ fixo é igual a

$$
\left(\begin{array}{cc}
\mathbf{K}_{p}^{\boldsymbol{\beta} \boldsymbol{\beta}} & \mathbf{K}_{p}^{\boldsymbol{\beta} \mathbf{f}} \\
\mathbf{K}_{p}^{\mathbf{f} \boldsymbol{\beta}} & \mathbf{K}_{p}^{\mathrm{ff}}
\end{array}\right)^{(u)}\left(\begin{array}{c}
\boldsymbol{\beta}^{(u+1)}-\boldsymbol{\beta}^{(u)} \\
\mathbf{f}^{(u+1)}-\mathbf{f}^{(u)}
\end{array}\right)=\left(\begin{array}{c}
\mathbf{U}_{p}^{\boldsymbol{\beta}} \\
\mathbf{U}_{p}^{\mathbf{f}}
\end{array}\right)^{(u)}
$$

Assim, com os valores obtidos anteriormente (3.4) pode ser escrita como

$$
\left(\begin{array}{c}
\boldsymbol{\beta}^{(u+1)} \\
\mathbf{f}^{(u+1)}
\end{array}\right)=\left(\begin{array}{c}
\boldsymbol{\beta}^{(u)} \\
\mathbf{f}^{(u)}
\end{array}\right)+\left(\begin{array}{cc}
\mathbf{X}^{T} \mathbf{W X} & \mathbf{X}^{T} \mathbf{W} \mathbf{N} \\
\mathbf{N}^{T} \mathbf{W X} & \mathbf{N}^{T} \mathbf{W} \mathbf{N}+\lambda \mathbf{K}
\end{array}\right)^{(u)}\left(\begin{array}{c}
\mathbf{X}^{T} \mathbf{W} \mathbf{z} \\
\mathbf{N}^{T} \mathbf{W} \mathbf{z}-\lambda \mathbf{K} \mathbf{f}
\end{array}\right)^{(u)}
$$

Com algumas manipulações algébricas é possível escrever as equações de estimação

$$
\begin{aligned}
\mathbf{f}^{(u+1)} & =\left(\mathbf{N}^{T} \mathbf{W}^{(u)} \mathbf{N}+\lambda \mathbf{K}\right)^{-1} \mathbf{N}^{T} \mathbf{W}^{(u)}\left(\mathbf{z}^{(u)}-\mathbf{X} \boldsymbol{\beta}^{(u)}\right) \mathrm{e} \\
\boldsymbol{\beta}^{(u+1)} & =\left(\mathbf{X}^{T} \mathbf{W}^{(u)} \mathbf{X}\right)^{-1} \mathbf{X}^{T} \mathbf{W}^{(u)}\left(\mathbf{z}^{(u)}-\mathbf{N} \mathbf{f}^{(u)}\right)
\end{aligned}
$$

em que $\mathbf{z}=\mathbf{F}^{-1}(\mathbf{y}-\boldsymbol{\mu})+\mathbf{X} \boldsymbol{\beta}+\mathbf{N f}$.

Intuitivamente podemos observar que, se $\boldsymbol{\beta}$ é conhecido e considerado constante em $(\mathbf{z}-\mathbf{X} \boldsymbol{\beta}),(3.11)$ nos diz à respeito do ajuste do spline cúbico via máxima verossimilhança penalizada das diferenças ( $\mathbf{z}-\mathbf{X} \boldsymbol{\beta})$. Da mesma forma, se $\mathbf{f}$ for conhecido, (3.12) é uma estimativa de $\boldsymbol{\beta}$ via máxima verossimilhança penalizada das diferenças $(\mathbf{z}-\mathbf{N f})$. Essa interpretação pode ser levada adiante, no sentido que podemos fazer repetidamente uma alternância entre (3.11) e (3.12) para obter $\hat{\boldsymbol{\beta}}$ e $\hat{\mathbf{f}}$ via máxima verossimilhança penalizada até a convergência desejada. Esse algoritmo é conhecido como backfitting, e foi introduzido por Breiman e Friedman (1985). Mais detalhes referentes a este algoritmo, consultar Green et al. (1985) ou Buja et al. (1989).

Note que os parâmetros $\left(\boldsymbol{\beta}^{T}, \mathbf{f}^{T}\right)^{T}$ e $\phi$ são ortogonais, assim podemos desenvolver um processo iterativo para $\phi$ dadas as estimativas de $\boldsymbol{\beta}$ e $\mathbf{f}$, expresso na forma abaixo

$$
\phi^{(s+1)}=\phi^{(s)}+\left\{\mathrm{U}_{p}^{\phi} / \ddot{\mathrm{L}}_{p}^{\phi \phi}\right\}^{(s)}
$$

para $s=0,1, \ldots$, em que $\mathrm{U}_{p}^{\phi}$ e $\ddot{\mathrm{L}}_{p}^{\phi \phi}$ denotam, respectivamente, a função escore e a informação observada de Fisher para $\phi$. O processo iterativo (3.11)-(3.12) deve alternar com o processo iterativo (3.13).

Portanto, o processo iterativo para estimar $\boldsymbol{\beta}, \mathbf{f}$ e $\phi$ pode ser resumido nos passos abaixo Passo 1: atribuimos valores iniciais para $\boldsymbol{\beta}, \mathbf{f}$ e $\phi$. Por exemplo, podemos considerar as 
estimativas de $\boldsymbol{\beta}$ e $\phi$ do modelo binomial negativo paramétrico e atribuir $\mathbf{f}^{(0)}=0$;

Passo 2: supondo $\phi$ fixado ( $\lambda$ já fixado), aplicar o processo iterativo (3.11)-(3.12) até a convergência;

Passo 3: dadas as estimativas obtidas para $\boldsymbol{\beta}$ e $\mathbf{f}$ no Passo 2, aplicar o processo iterativo (3.13) até a convergência;

Passo 4: repetir os Passos 2 e 3 até a convergência.

Green e Silverman (1994) provam que, sob determinadas condições relacionadas às propriedades das matrizes $\mathbf{N}, \mathbf{W}$ e $\mathbf{K}$, o algoritmo backfitting sempre converge e pode terminar em pouco mais de 5 iterações.

\subsubsection{Graus de liberdade efetivos}

Um tópico importante em modelagem semiparamétrica é estimar o número de graus de liberdade efetivos, isto é, quantos graus de liberdade estão efetivamente sendo utilizados para estimar os parâmetros do componente não paramétrico. No caso dos modelos semiparamétricos com resposta binomial negativa o número total de parâmetros para serem estimados é dado por $q=1+p+d f(\lambda)$, em que $p+1$ referem-se aos parâmetros $\boldsymbol{\beta}$ e $\phi$, enquanto $d f(\lambda)$ aos parâmetros do componente não paramétrico. Em Green e Silverman (1994) há uma expressão para $d f(\lambda)$ para os MLGs que pode ser adaptada para os modelos semiparamétricos com resposta binomial negativa em virtude da semelhança entre as equações de estimação. Essa expressão pode ser escrita na forma

$$
d f(\lambda)=\operatorname{tr}\{\mathbf{H}(\lambda)\}-\operatorname{tr}\left\{\mathbf{H}_{1}(\lambda)\right\}
$$

em que

$$
\mathbf{H}(\lambda)=\mathbf{N}\left(\mathbf{N}^{T} \mathbf{W} \mathbf{N}+\lambda \mathbf{K}\right)^{-1} \mathbf{N}^{T} \mathbf{W}
$$

$\mathrm{e}$

$$
\mathbf{H}_{1}(\lambda)=\left[\mathbf{X}^{T} \mathbf{W}\left\{\mathbf{I}_{n}-\mathbf{H}(\lambda)\right\} \mathbf{X}\right]^{-1} \mathbf{X}^{T} \mathbf{W}\left\{\mathbf{I}_{n}-\mathbf{H}(\lambda)\right\}^{2} \mathbf{X}
$$

com $\mathbf{I}_{n}$ sendo a matriz identidade de ordem $\mathrm{n}$.

No Capítulo 4 derivamos uma outra expressão para $d f(\lambda)$ através de um modelo linear equivalente discutido por Noda (2013, Cap. 3). A expressão para $d f(\lambda)$ assume a forma

$$
d f(\lambda)=\operatorname{tr}\{\mathbf{H}(\lambda)\}-\operatorname{tr}\{\mathbf{H}(\lambda) \widetilde{\mathbf{H}}(\lambda)\}
$$

em que

$$
\widetilde{\mathbf{H}}(\lambda)=\left\{\mathbf{I}_{n}-\mathbf{H}(\lambda)\right\}^{-1} \widetilde{\mathbf{X}}\left(\widetilde{\mathbf{X}}^{T} \mathbf{W} \widetilde{\mathbf{X}}\right)^{-1} \widetilde{\mathbf{X}}^{T} \mathbf{W}\left\{\mathbf{I}_{n}-\mathbf{H}(\lambda)\right\},
$$

e $\widetilde{\mathbf{X}}=\left\{\mathbf{I}_{n}-\mathbf{H}(\lambda)\right\} \mathbf{X}$ 


\subsubsection{Estimação do parâmetro de suavização}

A estimação do parâmetro de suavização $\lambda$ consiste em uma etapa crucial no processo de estimação dos parâmetros $\boldsymbol{\beta}, \mathbf{f}$ e $\phi$ e há alguns procedimentos propostos. Por exemplo, a utilização do escore denominado validação cruzada generalizada que é basicamente uma medida de qualidade de ajuste para $\lambda$ fixado. Em Wood (2006, Cap. 4) é apresentada uma proposta para MLGs que pode ser adaptada para os modelos semiparamétricos com resposta binomial negativa. Essa expressão é dada por

$$
V C G(\lambda)=\frac{\sum_{i=1}^{n} \hat{w}_{i}\left(\hat{z}_{i}-x_{i}^{T} \hat{\boldsymbol{\beta}}\right)^{2}}{\{n-d f(\lambda)\}^{2}}
$$

em que as estimativas são obtidas através do processo iterativo (3.11)-(3.13). Deve-se escolher o valor de $\lambda$ que minimize o escore $V C G(\lambda)$.

Outra possibilidade consiste na aplicação de medidas de informação, tais como os critérios de Akaike (1974) e Schwarz (1978) adaptados ao caso semiparamétrico. Assim, temos as quantidades

$$
A I C(\lambda)=-\mathrm{L}_{p}(\hat{\boldsymbol{\theta}}, \lambda)-\{p+d f(\lambda)\}
$$

e

$$
B I C(\lambda)=-\mathrm{L}_{p}(\hat{\boldsymbol{\theta}}, \lambda)-\{p+d f(\lambda)\} \log (n),
$$

em que $\mathrm{L}_{p}(\hat{\boldsymbol{\theta}}, \lambda)$ denota o logaritmo da função de verossimilhança penalizada avaliada em $\hat{\boldsymbol{\theta}}$. Como é desejável que $d f(\lambda)$ seja o menor possível, uma sugestão é procurar aproximadamente o valor de $\lambda$ que minimize $A I C(\lambda)$ (ou $B I C(\lambda)$ ) e $d f(\lambda)$.

\subsection{Resultados assintóticos}

Como o modelo semiparamétrico com resposta binomial negativa proposto neste trabalho é uma extensão do modelo com resposta binomial negativa usual, é razoável supor que, sob as condições de regularidade, as estimativas de máxima verossimilhança de $\boldsymbol{\theta}$ são assintoticamente normais, ou seja, podemos supor que $\hat{\boldsymbol{\theta}} \sim \mathrm{N}_{q}\left(\boldsymbol{\theta}, \mathbf{K}_{\boldsymbol{\theta} \boldsymbol{\theta}}^{-1}\right)$. Contudo é necessário supor que a função $f(t)$ está sendo consistentemente estimada por $\hat{f}(t)$.

Por meio da matriz de informação de Fisher penalizada calculada na Seção 3.3.3 e de propriedades de inversa de matriz em blocos, é possível mostrar que $\mathbf{K}_{\boldsymbol{\theta} \boldsymbol{\theta}}^{-1}$ pode ser expressa da seguinte forma:

$$
\left[\mathbf{K}_{p}^{\boldsymbol{\theta} \boldsymbol{\theta}}\right]^{-1}=\left(\begin{array}{ccc}
\mathbf{A} & \mathbf{B} & \mathbf{0} \\
\mathbf{C} & \mathbf{D} & \mathbf{0} \\
\mathbf{0} & \mathbf{0} & \mathrm{K}_{\phi \phi}^{-1}
\end{array}\right)
$$


em que

$$
\begin{aligned}
& \mathbf{A}=\left\{\mathbf{K}_{p}^{\boldsymbol{\beta} \boldsymbol{\beta}}-\mathbf{K}_{p}^{\mathbf{f} \boldsymbol{\beta}}\left[\mathbf{K}_{p}^{\mathrm{ff}}\right]^{-1} \mathbf{K}_{p}^{\boldsymbol{\beta} \mathbf{f}}\right\}^{-1} \\
& \mathbf{B}=-\left[\mathbf{K}_{p}^{\boldsymbol{\beta} \boldsymbol{\beta}}\right]^{-1} \mathbf{K}_{p}^{\mathbf{f} \boldsymbol{\beta}}\left\{\mathbf{K}_{p}^{\mathrm{ff}}-\mathbf{K}_{p}^{\boldsymbol{\beta} \mathbf{f}}\left[\mathbf{K}_{p}^{\boldsymbol{\beta} \boldsymbol{\beta}}\right]^{-1} \mathbf{K}_{p}^{\mathbf{f} \boldsymbol{\beta}}\right\}^{-1} \\
& \mathbf{C}=-\left[\mathbf{K}_{p}^{\mathrm{ff}}\right]^{-1} \mathbf{K}_{p}^{\boldsymbol{\beta f}}\left\{\mathbf{K}_{p}^{\boldsymbol{\beta} \boldsymbol{\beta}}-\mathbf{K}_{p}^{\mathbf{f} \boldsymbol{\beta}}\left[\mathbf{K}_{p}^{\mathrm{ff}}\right]^{-1} \mathbf{K}_{p}^{\boldsymbol{\beta f}}\right\}^{-1} \mathrm{e} \\
& \mathbf{D}=\left\{\mathbf{K}_{p}^{\mathrm{ff}}-\mathbf{K}_{p}^{\boldsymbol{\beta} \mathbf{f}}\left[\mathbf{K}_{p}^{\boldsymbol{\beta} \boldsymbol{\beta}}\right]^{-1} \mathbf{K}_{p}^{\mathbf{f} \boldsymbol{\beta}}\right\}^{-1} .
\end{aligned}
$$

Podemos notar que $\left(\boldsymbol{\beta}^{T}, \mathbf{f}^{T}\right)^{T}$ é ortogonal a $\phi$, assim $\hat{\boldsymbol{\beta}}$ e $\hat{\mathbf{f}}$ podem apresentar alguma correlação assintótica. É usual em modelos semiparamétricos construir uma banda de confiança para a função não paramétrica $f(t)$. Essa banda é usualmente construída através da junção das estimativas intervalares

$$
\hat{f}\left(t_{i}\right) \pm z_{(1-\alpha / 2)} \sqrt{\widehat{\operatorname{Var}}\left\{\hat{f}\left(t_{i}\right)\right\}}
$$

em que $z_{(1-\alpha / 2)}$ denota o percentil $(1-\alpha / 2)$ da distribuição $\mathrm{N}(0,1)$.

\subsection{Considerações}

Neste capítulo apresentamos alguns resultados de estimação e inferência referentes aos modelos semiparamétricos com resposta binomial negativa. Derivamos as funções escore penalizadas, bem como as matrizes de Fisher observada e esperada penalizadas, assim como a estimação dos componentes paramétricos e não paramétrico pela maximização da verossimilhança penalizada, estimação do parâmetro de suavização e aspectos inferênciais. 


\section{Capítulo 4}

\section{Métodos de diagnóstico}

Uma das etapas mais importantes da modelagem estatística é a análise de diagnóstico. Quando trabalhamos com análise de dados, geralmente os modelos de regressão procuram captar relações entre a variável resposta controlando o efeito de variáveis explicativas. No entanto, é muito difícil conhecer o modelo verdadeiro na prática, o que faz com que o modelo proposto nem sempre se adeque bem aos dados. Assim, a análise de diagnóstico do modelo ajustado tem como principal objetivo verificar a sua adequação. Em geral, procurase saber se os valores ajustados estão muito longe dos valores reais, para detecção de pontos aberrantes, bem como verificar se há afastamentos importantes das suposições do modelo como a distribuição dos erros. Uma outra verificação importante é a análise de influência das observações, para detectar se alguma observação está influenciando as estimativas dos parâmetros do modelo de forma desproporcional e com mudanças inferenciais.

Uma medida que é muito utilizada e conhecida para detectar pontos influentes é a distância de Cook (1977). Originalmente foi desenvolvida para modelos normais lineares e rapidamente assimilada e estendida para várias classes de modelos. Porém, uma das propostas mais inovadoras na área de diagnóstico em regressão foi apresentada por Cook (1986). Ao invés da avaliação pela retirada individual ou conjunta de pontos, foi proposto avaliar a influência conjunta das observações sob pequenas mudanças, chamadas perturbações locais, no modelo ou nos dados. Denominada influência local, essa metodologia teve uma grande receptividade entre os usuários e pesquisadores de regressão, havendo inúmeras publicações no assunto em que a metodologia é aplicada em classes particulares de modelos ou estendida para situações mais gerais, assim como neste trabalho.

Neste capítulo, apresentamos algumas técnicas de diagnóstico para o modelo proposto (3.1). Inicialmente faremos derivações de medidas de alavanca para o modelo semiparamétrico com resposta binomial negativa usando um modelo linear equivalente, definiremos um resíduo componente do desvio padronizado, faremos também derivações de distâncias de Cook e concluiremos o capítulo aplicando a metodologia de influência local. 


\subsection{Matrizes de alavancas}

Esta seção está destinada à apresentar uma forma de obter o grau de alavanca das observações, assim como permitir avaliar tais quantidades. O grau de alavanca é utilizado para detectar se a observação está influenciando de forma desproporcional na sua própria estimativa.

Como foi observado na Seção 2.4 a definição de alavanca na $i$-ésima observação (que consiste da derivada $\left.\partial \hat{y}_{i} / \partial y_{i}\right)$ somente pode ser aplicada em modelos com resposta contínua. Assim, também para o caso semiparamétrico teremos que obter medidas indiretas através de modelos lineares semiparamétricos equivalentes. Desta forma, note que na convergência do processo iterativo (3.11)-(3.12) obtemos as equações

$$
\hat{\mathbf{f}}=\left(\mathbf{N}^{T} \widehat{\mathbf{W}} \mathbf{N}+\lambda \mathbf{K}\right)^{-1} \mathbf{N}^{T} \widehat{\mathbf{W}}(\hat{\mathbf{z}}-\mathbf{X} \hat{\boldsymbol{\beta}})
$$

e

$$
\hat{\boldsymbol{\beta}}=\left(\mathbf{X}^{T} \widehat{\mathbf{W}} \mathbf{X}\right)^{-1} \mathbf{X}^{T} \widehat{\mathbf{W}}(\hat{\mathbf{z}}-\mathbf{N} \hat{\mathbf{f}})
$$

em que $\hat{\mathbf{z}}=\widehat{\mathbf{F}}^{-1}(\mathbf{y}-\hat{\boldsymbol{\mu}})+\mathbf{X} \hat{\boldsymbol{\beta}}+\mathbf{N} \hat{\mathbf{f}} \operatorname{com} \hat{\boldsymbol{\mu}}=\mathbf{X} \hat{\boldsymbol{\beta}}+\mathbf{N} \hat{\mathbf{f}}$.

Seguindo a notação descrita em Noda (2013, Cap. 3) as equações (4.1)-(4.2) coincidem com as soluções de mínimos quadrados penalizados do seguinte modelo linear:

$$
\hat{\mathbf{z}}=\mathbf{X} \boldsymbol{\beta}+\mathbf{N f}+\epsilon
$$

em que $\epsilon \sim N_{n}\left(\mathbf{0}, \widehat{\mathbf{W}}^{-1}\right)$. Assim, seguindo a mesma notação de Noda (2013, Cap. 3), a matriz de alavancas para o modelo (4.3) pode ser expressa na forma

$$
\widehat{\overline{\mathbf{H}}}(\lambda)=\widehat{\widetilde{\mathbf{H}}}(\lambda)+\widehat{\mathbf{H}}^{*}(\lambda),
$$

em que $\widehat{\widetilde{\mathbf{H}}}(\lambda)$ denota a matriz de alavancas para o componente paramétrico e $\widehat{\mathbf{H}}^{*}(\lambda)=$ $\widehat{\mathbf{H}}(\lambda)\left\{\mathbf{I}_{n}-\widehat{\widetilde{\mathbf{H}}}(\lambda)\right\}$ denota a matriz de alavancas para o componente não paramétrico sendo $\mathbf{I}_{n}$ a matriz identidade de ordem $n$.

Essas matrizes, fazendo uma adaptação para o modelo semiparamétrico com resposta binomial negativa, ficam expressas nas seguintes formas:

$$
\widetilde{\mathbf{H}}(\lambda)=\left\{\mathbf{I}_{n}-\mathbf{H}(\lambda)\right\}^{-1} \widetilde{\mathbf{X}}\left(\widetilde{\mathbf{X}}^{T} \mathbf{W} \widetilde{\mathbf{X}}\right)^{-1} \widetilde{\mathbf{X}}^{T} \mathbf{W}\left\{\mathbf{I}_{n}-\mathbf{H}(\lambda)\right\}
$$

e

$$
\mathbf{H}(\lambda)=\mathbf{N}\left(\mathbf{N}^{T} \mathbf{W} \mathbf{N}+\lambda \mathbf{K}\right)^{-1} \mathbf{N}^{T} \mathbf{W}
$$

$\operatorname{com} \widetilde{\mathbf{X}}=\left\{\mathbf{I}_{n}-\mathbf{H}(\lambda)\right\} \mathbf{X}$.

Denotando por $\hat{\bar{h}}_{i i}, \hat{\tilde{h}}_{i i}$ e $\hat{h}_{i i}^{*}$ os elementos das diagonais principais das matrizes $\widehat{\hat{\mathbf{H}}}(\lambda)$, $\widehat{\widetilde{\mathbf{H}}}(\lambda)$ e $\widehat{\mathbf{H}}^{*}(\lambda)$, respectivamente, os gráficos de índices dessas quantidades podem revelar 
observações influentes nos valores preditos do modelo semiparamétrico e nos valores preditos do componente paramétrico e nos valores preditos do componente não paramétrico, respectivamente.

\subsection{Resíduos}

A análise de resíduos é um dos procedimentos mais utilizados para avaliar se há afastamentos importantes das suposições feitas para o modelo e detectar pontos aberrantes. $\mathrm{O}$ resíduo componente do desvio procura medir a discrepância entre o valor observado e o ajustado de cada indivíduo.

Fazendo um paralelo com o modelo com resposta binomial negativa paramétrico, o resíduo componente do desvio para o modelo semiparamétrico binomial negativa fica dado por

$$
t_{D_{i}}=\frac{d^{*}\left(y_{i} ; \hat{\mu}_{i}\right)}{\sqrt{1-\hat{\bar{h}}_{i i}}}
$$

em que

$$
\mathrm{d}^{*}\left(y_{i} ; \hat{\mu}_{i}\right)= \begin{cases} \pm \sqrt{2}\left[\phi \log \left\{\frac{\hat{\mu}_{i}+\phi}{y_{i}+\phi}\right\}+y_{i} \log \left\{\frac{y_{i}\left(\hat{\mu}_{i}+\phi\right)}{\hat{\mu}_{i}\left(y_{i}+\phi\right)}\right\}\right]^{1 / 2} & \text { se } y_{i}>0 \\ \pm \sqrt{2} \phi \log \left\{\frac{\hat{\mu}_{i}+\phi}{\phi}\right\} & \text { se } y_{i}=0\end{cases}
$$

e $\hat{\mu}_{i}=\mathrm{g}^{-} 1\left(\mathrm{x}_{i}^{T} \hat{\boldsymbol{\beta}}+\mathrm{n}_{i}^{T} \hat{\mathbf{f}}\right)$.

Podemos construir, com esse resíduo, gráficos normais de probabilidades com banda de confiança simulada, a fim de verificarmos a adequação da distribuição dos erros bem como a presença de pontos aberrantes.

\subsection{Distância de Cook}

O desenvolvimento de medidas de influência para avaliar a influência da retirada individual de observações nas estimativas $\hat{\boldsymbol{\beta}}, \hat{\mathbf{f}}$ e $\hat{\boldsymbol{\mu}}$ podem ficar elaboradas em modelos semiparamétricos com resposta binomial negativa, em virtude de várias operações matriciais mais complexas. Assim, analogamente ao desenvolvimento de medidas de alavanca e fazendo uma adaptação dos resultados desenvolvidos por Noda (2013, Cap. 3), derivamos a seguir as expressões das distâncias de Cook para o modelo linear descrito em (4.3). Temos, portanto, três situações descritas a seguir. 


\section{Influência em $\hat{\boldsymbol{\beta}}$}

Neste caso a influência da retirada da $i$-ésima observação na estimativa $\hat{\boldsymbol{\beta}}$ fica dada por

$$
\tilde{D}_{i}=\left\{\frac{\hat{\tilde{r}}_{i}}{1-\hat{\tilde{h}}_{i i}}\right\}^{2} \frac{\hat{\tilde{h}}_{i i}}{p}
$$

em que $\tilde{r}_{i}$ é o $i$-ésimo componente do vetor de resíduos

$$
\tilde{\mathbf{r}}=\left\{\mathbf{I}_{n}-\tilde{\mathbf{H}}(\lambda)\right\} \hat{\mathbf{z}}
$$

\section{Influência em $\hat{\mathbf{f}}$}

Aqui a influência da retirada da $i$-ésima observação na estimativa $\hat{\mathbf{f}}$ fica dada por

$$
D_{i}^{*}=\left\{\frac{\hat{r}_{i}^{*}}{1-\hat{h}_{i i}^{*}}\right\}^{2} \frac{\hat{h}_{i i}^{*}}{d f(\lambda)}
$$

em que $r_{i}^{*}$ é o $i$-ésimo componente do vetor de resíduos

$$
\mathbf{r}^{*}=\left\{\mathbf{I}_{n}-\mathbf{H}^{*}(\lambda)\right\} \hat{\mathbf{z}}
$$

e $d f(\lambda)$ é uma estimativa dos graus de liberdade efetivos no modelo semiparamétrico (4.3), sendo dada por

$$
d f(\lambda)=\operatorname{tr}\left\{\mathbf{H}^{*}(\lambda)\right\}
$$

\section{Influência em $\hat{\boldsymbol{\mu}}$}

Para avaliar a influência da retirada da $i$-ésima observação na média ajustada $\hat{\boldsymbol{\mu}}=$ $\mathbf{X} \hat{\boldsymbol{\beta}}+\mathbf{N} \hat{\mathbf{f}}$ do modelo (4.3), podemos aplicar a distância de Cook

$$
\bar{D}_{i}=\left\{\frac{\hat{\bar{r}}_{i}}{1-\hat{\bar{h}}_{i i}}\right\}^{2} \frac{\hat{\bar{h}}_{i i}}{p+d f(\lambda)},
$$

em que $\bar{r}_{i}$ é o $i$-ésimo componente do vetor de resíduos

$$
\overline{\mathbf{r}}=\left\{\mathbf{I}_{n}-\overline{\mathbf{H}}(\lambda)\right\} \hat{\mathbf{z}}
$$

Portanto, gráficos de índices das quantidades $\tilde{D}_{i}, D_{i}^{*}$ e $\bar{D}_{i}$ podem revelar a influência da retirada da $i$-ésima observação, respectivamente, nas estimativas $\hat{\boldsymbol{\beta}}, \hat{\mathbf{f}}$ e $\hat{\boldsymbol{\mu}}$. 


\subsection{Graus de liberdade efetivos}

Podemos alternativamente estimar os graus de liberdade do modelo semiparamétrico com resposta binomial negativa através do modelo linear semiparamétrico dado em (4.3). O total de parâmetros sendo estimado é dado por $1+p+d f(\lambda)$, em que

$$
\begin{aligned}
d f(\lambda) & =\operatorname{tr}\left\{\mathbf{H}^{*}(\lambda)\right\}=\operatorname{tr}\{\mathbf{H}(\lambda)-\mathbf{H}(\lambda) \widetilde{\mathbf{H}}(\lambda)\} \\
& =\operatorname{tr}\{\mathbf{H}(\lambda)\}-\operatorname{tr}\{\mathbf{H}(\lambda) \widetilde{\mathbf{H}}(\lambda)\}
\end{aligned}
$$

são os graus de liberdade efetivos para estimar o componente não paramétrico.

\subsection{Influência local}

A fim de detectarmos observações influentes, quando o modelo ou os dados são localmente perturbados, utilizaremos alguns esquemas de pertubações (Cook, 1986) adaptados aos modelos semiparamétricos com resposta binomial negativa. Valores altos para as medidas de influência local podem indicar alta sensibilidade das estimativas sob o esquema de perturbação adotado.

A ideia de influência local é definir um esquema de perturbação apropriado, por exemplo representado por $\delta=\left(\delta_{1}, \ldots, \delta_{n}\right)^{T}$, em que as perturbações são aplicadas no logaritmo da função de verossimilhança penalizada ou nos dados. Então devemos derivar a curvatura normal numa direção unitária $\mathbf{l}$ da superfície $\left(\delta^{T}, \mathbf{L D}(\delta)\right)^{T}$, em que

$$
\mathbf{L D}(\delta)=2\left\{\mathrm{~L}_{p}(\hat{\boldsymbol{\theta}}, \lambda)-\mathrm{L}_{p}\left(\hat{\boldsymbol{\theta}}_{\delta}, \lambda\right)\right\}
$$

denota a medida de influência afastamento pela verossimilhança e $\mathrm{L}_{p}\left(\hat{\boldsymbol{\theta}}_{\delta}, \lambda\right)$ denota o logaritmo da função de verossimilhança penalizada avaliado na estimativa de máxima verossimilhança penalizada $\hat{\boldsymbol{\theta}}_{\delta}$ do modelo perturbado, ou seja, maximizando $\mathrm{L}_{p}(\boldsymbol{\theta}, \lambda \mid \delta)$.

A curvatura normal na direção unitária l, que é avaliada em $\hat{\boldsymbol{\theta}}$ e no vetor de não perturbação $\delta_{0}$, fica dada por

$$
\mathbf{C}_{\mathbf{l}}(\boldsymbol{\theta})=2\left|\mathbf{l}^{T} \boldsymbol{\Delta}^{T}\left(-\ddot{\mathrm{L}}_{\hat{\boldsymbol{\theta}} \hat{\boldsymbol{\theta}}}\right)^{-1} \boldsymbol{\Delta} \mathbf{l}\right|,
$$

em que $-\ddot{\mathbf{L}}_{\hat{\boldsymbol{\theta}} \hat{\boldsymbol{\theta}}}$ é a matriz de informação observada de Fisher penalizada correspondente a $\boldsymbol{\theta}=\left(\boldsymbol{\beta}^{T}, \mathbf{f}^{T}, \phi\right)^{T}$ e avaliada em $\hat{\boldsymbol{\theta}}$ e $\boldsymbol{\Delta}$ é um matriz $(q \times n)$, em que $q=1+p+h$ cujos elementos são dados por

$$
\boldsymbol{\Delta}_{j i}=\left.\frac{\partial^{2} \mathrm{~L}_{p}(\boldsymbol{\theta}, \lambda \mid \boldsymbol{\delta})}{\partial \theta_{j} \partial \delta_{i}}\right|_{\hat{\boldsymbol{\theta}, \delta_{0}}},
$$

para $j=1, \ldots, q$ e $i=1, \ldots, n$.

A sugestão original de Cook (1986) é considerar a direção em que a curvatura normal é 
maximizada, $C_{\mathbf{l}_{\text {max }}}(\boldsymbol{\theta})$, que fica dada por

$$
C_{\mathbf{l}_{\text {max }}}(\boldsymbol{\theta})=2\left|\mathbf{l}_{\max }^{T} \boldsymbol{\Delta}^{T}\left(-\ddot{\mathrm{L}}_{\hat{\boldsymbol{\theta}} \hat{\boldsymbol{\theta}}}\right)^{-1} \boldsymbol{\Delta} \mathbf{l}_{\max }\right| .
$$

Como $C_{\mathbf{l}_{\max }}$ corresponde ao maior autovalor da matriz $\mathbf{A}=\boldsymbol{\Delta}^{T}\left(-\ddot{\mathrm{L}}_{\hat{\boldsymbol{\theta}} \hat{\boldsymbol{\theta}}}\right)^{-1} \boldsymbol{\Delta}$ em valor absoluto, a sugestão de Cook é considerar o gráfico de índices do autovetor correspondente $\mathbf{l}_{\text {max }}$. Observações relativamente com os maiores valores devem ser destacadas como possivelmente influentes em $\hat{\boldsymbol{\theta}}$.

Uma variante da curvatura normal, ao invés de considerar a direção de maior variação, é considerar a direção de cada observação. Assim, tem-se a curvatura normal

$$
C_{i}(\boldsymbol{\theta})=2\left|\mathbf{l}_{i}^{T} \boldsymbol{\Delta}^{T}\left(-\ddot{\mathrm{L}}_{\hat{\boldsymbol{\theta}} \hat{\boldsymbol{\theta}}}\right)^{-1} \boldsymbol{\Delta} \mathbf{l}_{i}\right|,
$$

em que $\mathbf{l}_{i}$ é um vetor $(n \times 1)$ de zeros com 1 na $i$-ésima posição. Gráficos de índices de $C_{i}(\boldsymbol{\theta})$ podem ser construídos para avaliar a influência das observações em $\hat{\boldsymbol{\theta}}$. Um critério descritivo é destacar as observações tais que $C_{i}>2 \bar{C}$ em que $\bar{C}=\sum_{i=1}^{n} C_{i} / n$.

Podemos particionar o vetor $\boldsymbol{\theta}$ tal que $\boldsymbol{\theta}=\left(\boldsymbol{\theta}_{1}^{T}, \boldsymbol{\theta}_{2}^{T}\right)^{T}$ com o interesse sendo calcular a curvatura normal somente referente a $\boldsymbol{\theta}_{1}$, que poderia ser no modelo semiparamétrico com resposta binomial negativa, por exemplo $\boldsymbol{\theta}_{1}=\boldsymbol{\beta}, \boldsymbol{\theta}_{1}=\mathbf{f}$ ou $\boldsymbol{\theta}_{1}=\phi$. A curvatura normal neste caso, na direção unitária 1 , fica dada por

$$
C_{\mathbf{l}}\left(\boldsymbol{\theta}_{1}\right)=2\left|\mathbf{l}^{T} \boldsymbol{\Delta}^{T}\left(-\ddot{\mathrm{L}}_{\hat{\boldsymbol{\theta}} \hat{\boldsymbol{\theta}}}-\mathbf{B}_{1}\right)^{-1} \boldsymbol{\Delta} \mathbf{l}\right|
$$

em que

$$
\mathbf{B}_{1}=\left(\begin{array}{cc}
\mathbf{0} & \mathbf{0} \\
\mathbf{0} & -\ddot{\mathrm{L}}_{\hat{\boldsymbol{\theta}_{2}} \hat{\boldsymbol{\theta}_{2}}}
\end{array}\right)
$$

com $-\ddot{\mathrm{L}}_{\hat{\boldsymbol{\theta}_{2}} \hat{\boldsymbol{\theta}_{2}}}$ sendo a matriz de informação observada de Fisher penalizada de $\boldsymbol{\theta}_{2}$ e avaliada em $\hat{\boldsymbol{\theta}}$. Aqui devemos considerar o gráfico de índices do autovetor correspondente ao maior autovalor da matriz $\mathbf{B}=\boldsymbol{\Delta}^{T}\left(-\ddot{\mathrm{L}}_{\hat{\boldsymbol{\theta}} \hat{\boldsymbol{\theta}}}-\mathbf{B}_{1}\right)^{-1} \boldsymbol{\Delta}$. Similarmente, podemos considerar o gráfico de índices de $C_{i}\left(\boldsymbol{\theta}_{1}\right)$ para avaliar observações possivelmente influentes em $\boldsymbol{\theta}_{1}$ sob o esquema de perturbação adotado. A seguir derivamos a matriz $\Delta$ sob dois esquemas usuais de perturbação.

\subsubsection{Ponderação de casos}

Considere que cada observação possui um peso diferente no processo de estimação, assim podemos avaliar a contribuição individual de cada observação. Este esquema é um dos mais utilizados na prática. Neste caso, o logaritmo da função de verossimilhança penalizada fica 
dado por

$$
\mathrm{L}_{p}(\boldsymbol{\theta}, \lambda \mid \boldsymbol{\delta})=\sum_{i=1}^{n} \delta_{i} \mathrm{~L}_{i}(\boldsymbol{\theta})-\frac{\lambda}{2} \mathbf{f}^{T} \mathbf{K f},
$$

em que

$$
\mathrm{L}_{i}(\boldsymbol{\theta})=\frac{\Gamma\left(\phi+y_{i}\right)}{\Gamma\left(y_{i}+1\right) \Gamma(\phi)}\left(\frac{\mu_{i}}{\mu_{i}+\phi}\right)^{y_{i}}\left(\frac{\phi}{\mu_{i}+\phi}\right)^{\phi},
$$

$\boldsymbol{\delta}=\left(\delta_{1}, \ldots, \delta_{n}\right)^{T}$ é o vetor de pesos, com $0 \leq \delta_{i} \leq 1$. Neste esquema de perturbação, o vetor de não perturbação é dado por $\boldsymbol{\delta}_{0}=(1, \ldots, 1)^{T}$.

Podemos decompor a matriz $(q \times n) \boldsymbol{\Delta}$ em submatrizes $\boldsymbol{\Delta}=\left(\boldsymbol{\Delta}_{1}^{T}, \boldsymbol{\Delta}_{2}^{T}, \boldsymbol{\Delta}_{3}^{T}\right)^{T}$, em que $\boldsymbol{\Delta}_{1}, \boldsymbol{\Delta}_{2}$ e $\boldsymbol{\Delta}_{3}$ são submatrizes de dimensões $(p \times n),(h \times n)$ e $(1 \times n)$, respectivamente, e com elementos definidos por

$$
\boldsymbol{\Delta}_{1 j i}=\left.\frac{\partial^{2} \mathrm{~L}_{p}(\boldsymbol{\theta}, \lambda \mid \boldsymbol{\delta})}{\partial \beta_{j} \partial \delta_{i}}\right|_{\hat{\boldsymbol{\theta}}, \delta_{0}}
$$

para $j=1, \ldots, p$

$$
\boldsymbol{\Delta}_{2 j i}=\left.\frac{\partial^{2} \mathrm{~L}_{p}(\boldsymbol{\theta}, \lambda \mid \boldsymbol{\delta})}{\partial f_{j} \partial \delta_{i}}\right|_{\hat{\boldsymbol{\theta}, \delta_{0}}}
$$

para $j=1, \ldots, h$ e

$$
\boldsymbol{\Delta}_{3 i}=\left.\frac{\partial^{2} \mathrm{~L}_{p}(\boldsymbol{\theta}, \lambda \mid \boldsymbol{\delta})}{\partial \phi \partial \delta_{i}}\right|_{\hat{\boldsymbol{\theta}}, \delta_{0}}
$$

para $i=1, \ldots, n$. Após algumas manipulações algébricas (ver Apêndice C) obtemos $\boldsymbol{\Delta}_{1 j i}=$ $x_{i j} v_{i}$ para $j=1, \ldots, p, \Delta_{2 j i}=n_{i j} v_{i}$ para $j=1, \ldots, h$ e $\Delta_{3 i}=u_{i}$ para $i=1, \ldots, n$, em que $v_{i}=\left(w_{i} / f_{i}\right)\left(y_{i}-\mu_{i}\right), n_{i j}$ é o $i j$-ésimo elemento da matriz de incidência e $u_{i}=$ $\left[\psi\left(\phi+y_{i}\right)-\psi(\phi)-\frac{\left(y_{i}+\phi\right)}{\left(\phi+\mu_{i}\right)}+\log \left\{\frac{\phi}{\left(\phi+\mu_{i}\right)}\right\}+1\right]$. Podemos, então, expressar as submatrizes $\Delta_{1}, \Delta_{2}$ e $\Delta_{3}$ em forma matricial

$$
\begin{aligned}
& \boldsymbol{\Delta}_{1}=\mathbf{X}^{T} \mathbf{D}(\mathbf{v}) \\
& \boldsymbol{\Delta}_{2}=\mathbf{N}^{T} \mathbf{D}(\mathbf{v}) \quad \mathrm{e} \\
& \boldsymbol{\Delta}_{3}=\mathbf{1}^{T} \mathbf{D}(\mathbf{u}),
\end{aligned}
$$

em que $\mathbf{D}(v)$ e $\mathbf{D}(u)$ são matrizes diagonais $(n \times n)$ com elementos, respectivamente, dados por $\mathbf{v}=\left(v_{1}, \ldots, v_{n}\right)^{T}$ e $\mathbf{u}=\left(u_{1}, \ldots, u_{n}\right)^{T}$ e $\mathbf{1}$ é um vetor $(n \times 1)$ de uns.

Assim, podemos derivar várias expressões para a curvatura normal compondo as matrizes acima com a matriz de informação observada de Fisher penalizada $-\ddot{\mathrm{L}}_{\hat{\boldsymbol{\theta}} \hat{\boldsymbol{\theta}}}$ (avaliada em $\hat{\boldsymbol{\theta}}$ ) e/ou com as demais submatrizes obtidas na Seção 3.3.3. Gráficos apropriados poderão ser construídos para estudar a sensibilidade das estimativas $\hat{\boldsymbol{\theta}}, \hat{\boldsymbol{\beta}}, \hat{\mathbf{f}}$ e $\hat{\phi}$ sob o esquema de perturbação adotado, destacando as observações com maior contribuição nesta sensibilidade.

\subsubsection{Perturbação aditiva em uma variável explicativa contínua}

Nesta subseção consideramos uma perturbação aditiva em uma variável explicativa contínua do modelo. Assim, supomos que a $r$-ésima variável explicativa fique expressa por 
$x_{\delta_{i}}^{(r)}=x_{i}^{(r)}+\delta_{i}, i=1, \ldots, n$, em que $\delta_{i} \in \mathbb{R}$ e $\delta_{0}=(0, \ldots, 0)^{T}$.

Para este esquema de perturbação os componentes das submatrizes $\boldsymbol{\Delta}_{1}, \boldsymbol{\Delta}_{2}$ e $\boldsymbol{\Delta}_{3}$ ficam, respectivamente, dados por $\boldsymbol{\Delta}_{1 j i}=-x_{i j} w_{i} \beta_{r}, \boldsymbol{\Delta}_{2 j i}=-n_{i j} w_{i} \beta_{r}$ e $\boldsymbol{\Delta}_{3 i}=s_{i}$, em que $\beta_{r}$ representa o coeficiente da $r$-ésima variável explicativa (assumida contínua) do componente paramétrico e

$$
s_{i}=-\beta_{r} f_{i}\left\{\left(\phi+y_{i}\right)+\frac{1}{\left(\phi+\mu_{i}\right)}\right\}
$$

para $i=1, \ldots, n$. Em forma matricial essas submatrizes (ver Apêndice C) ficam expressas nas formas

$$
\begin{aligned}
\boldsymbol{\Delta}_{1} & =-\beta_{r} \mathbf{X}^{T} \mathbf{W}, \\
\boldsymbol{\Delta}_{2} & =-\beta_{r} \mathbf{N}^{T} \mathbf{W} \quad \mathrm{e} \\
\boldsymbol{\Delta}_{3} & =\mathbf{1}^{T} \mathbf{D}(\mathrm{s}),
\end{aligned}
$$

em que $\mathbf{D}(\mathbf{s})$ é uma matriz diagonal $(n \times n)$ com elementos dados por $\mathbf{s}=\left(s_{1}, \ldots, s_{n}\right)^{T}$. Portanto, fazendo a conjunção das matrizes $\Delta, \Delta_{1}, \Delta_{2}$ e $\Delta_{3}$ com as submatrizes da matriz de informação observada de Fisher penalizada $-\ddot{\mathrm{L}}_{\hat{\boldsymbol{\theta}} \hat{\boldsymbol{\theta}}}$ (avaliada em $\hat{\boldsymbol{\theta}}$ ), podemos derivar diversas curvaturas normais de influência local. Consequentemente, através de gráficos apropriados poderemos avaliar a sensibilidade das estimativas $\hat{\boldsymbol{\theta}}, \hat{\boldsymbol{\beta}}, \hat{\mathbf{f}}, \hat{\phi}$ sob o esquema de perturbação adotado, destacando aquelas observações com maior contribuição para esta sensibilidade.

\subsection{Considerações}

Este capítulo teve como principal objetivo derivar algumas medidas de diagnóstico para a verificação da adequabilidade do ajuste e para a detecção de possíveis pontos de alavanca e pontos influentes em modelos semiparamétricos com resposta binomial negativa. Uma versão para o resíduo componente do desvio também foi apresentada com o objetivo de avaliar as suposições para o modelo via gráfico normal de probabilidades bem como detectar pontos aberrantes. As medidas referentes à eliminação individual de pontos, bem como para detectar pontos de alavanca, foram adaptadas das medidas desenvolvidas por Noda (2013, Cap. 3) para modelos parcialmente lineares com erros normais heterocedásticos. Já as curvaturas normais de influência local foram obtidas diretamente para o modelo semiparamétrico com resposta binomial negativa sob esquemas usuais de perturbação. 


\section{Capítulo 5}

\section{Aplicação}

Neste capítulo é apresentada uma aplicação prática para exemplificar a teoria proposta neste trabalho. Para isso, serão analisados os dados apresentados no exemplo ilustrativo descrito no Capítulo 1. Os códigos em $\mathrm{R}$ desenvolvidos para a estimação dos parâmetros do modelo, assim como para construir a análise dos resíduos estão disponíveis no Apêndice D.

Para a análise desses dados aplicamos um modelo semiparamétrico com resposta binomial negativa, relacionando o número de ppm da máxima diária da concentração média de ozônio por hora (variável resposta) em Upland com as variáveis explicativas descritas no Cap. 1. Observamos por meio de análises descritivas que a covariável day tem um comportamento não linear em relação à variável resposta. Assim, vamos introduzir esta variável no modelo de forma não paramétrica. Após algumas análises de seleção de modelos chegamos ao seguinte modelo:

$$
\begin{aligned}
\text { O3 } & \sim \mathrm{BN}\left(\mu_{i}, \phi\right) \\
\log \left(\mu_{i}\right) & =\beta_{0}+\beta_{1} \text { humidity }_{i}+\beta_{2} \text { temp }_{i}+\beta_{3} \mathrm{ibh}_{i}+\beta_{4} \mathrm{ibt}_{i}+\beta_{5} \mathrm{vis}_{i}+f\left(\text { day }_{i}\right),
\end{aligned}
$$

para $i=1, \ldots, 330$, em que $\mu_{i}$ representa a média ppm de ozônio, $\phi$ é o parâmetro de forma, $f(\cdot)$ denota uma função não paramétrica e $\boldsymbol{\beta}=\left(\beta_{0} ; \beta_{1} ; \beta_{2} ; \beta_{3} ; \beta_{4} ; \beta_{5}\right)^{T}$ representa o vetor de parâmetros do componente paramétrico do modelo. O modelo (5.1) foi selecionado através do procedimento de Akaike.

Como discutido no Capítulo 3 o parâmetro de suavização $(\lambda)$ tem um importante papel no ajuste do modelo. Para escolhermos o valor de $\lambda$, optamos por analisar conjuntamente o valor de AIC e o critério BIC. Como os graus de liberdade têm relação direta com o parâmetro de suavização optamos por construir a Figura 5.1, em que apresentamos os valores de AIC e BIC para diferentes valores de graus de liberdade.

Podemos notar que há um ponto mínimo nos gráficos da Figura 5.1, ou seja, neste ponto que temos o grau de liberdade efetivo (ou o parâmetro de suavização), tal que minimiza os 


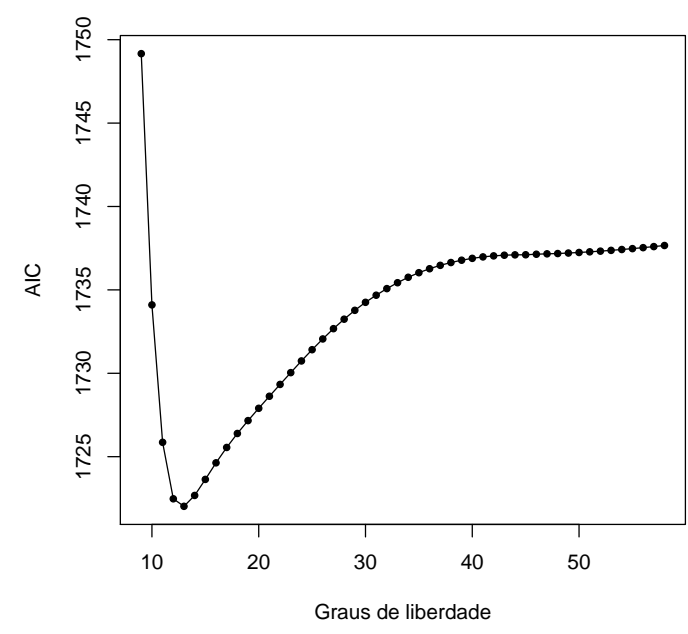

(a)

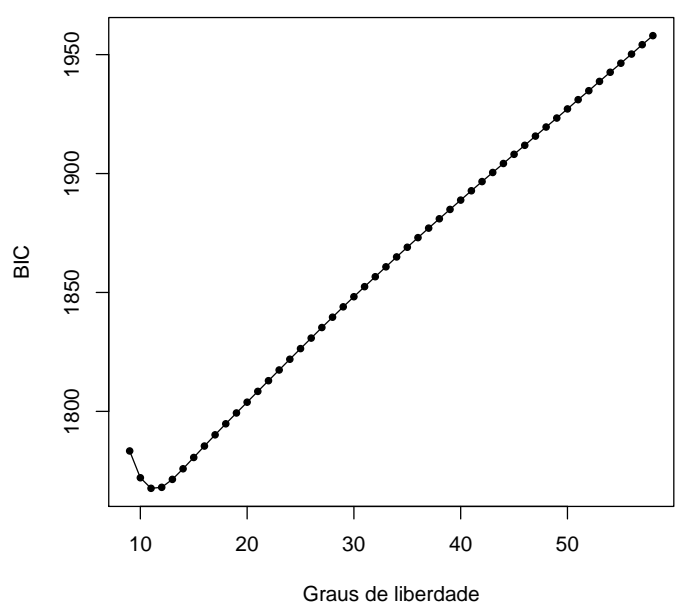

(b)

Figura 5.1: Valor de AIC para diferentes graus de liberdade efetivos 5.1(a), valor de BIC para diferentes graus de liberdade efetivos 5.1(b).

critérios. Podemos construir também um gráfico para observar o comportamento do parâmetro de suavização.

A Figura 5.2 mostra o comportamento do parâmetro de suavização conforme aumentamos os graus de liberdade efetivos. Nota-se que à medida que aumentamos os graus de liberdade o parâmetro de suavização diminui rapidamente, como esperado, se aproximando de zero a partir de 20 graus de liberdade aproximadamente. Decidimos escolher o grau de liberdade igual a 12, achando um meio termo entre os dois critérios.

Utilizando o software R (R Core Team, 2013) obtemos, através do critério de Akaike, a estimativa do parâmetro de suavização $\lambda$ sob o modelo proposto com 13 graus de liberdade dada por $\hat{\lambda}=0,003622$. Os erros padrão aproximados das estimativas dos componentes paramétricos e não paramétricos foram obtidos através da inversa da informação de Fisher penalizada, avaliada nas estimativas de máxima verossimilhança penalizadas descritas na Tabela 5.1. Não apresentamos as estimativas referentes à parte não paramétrica devido à grande quantidade de estimativas.

Nota-se pela Tabela 5.1 que todos os coeficientes são marginalmente significativos. À medida que aumenta a umidade, a temperatura ou a inversão de temperatura espera-se aumento para a concentração de ozônio, mantidas as demais variáveis explicativas fixas. Por outro lado, à medida que aumentam a altura da inversão de temperatura ou a visibilidade espera-se redução da concentração de ozônio, mantidas as demais variáveis explicativas fixas. O controle através da função não paramétrica da variável day reduz um pouco as estimativas dos erros padrão, principalmente na estimativa do parâmetro de dispersão para o qual houve uma redução considerável. Note que a inclusão da variável explicativa day de forma não paramétrica mostrou-se significativa. A Figura 5.3 descreve o ajuste dessa variável. 


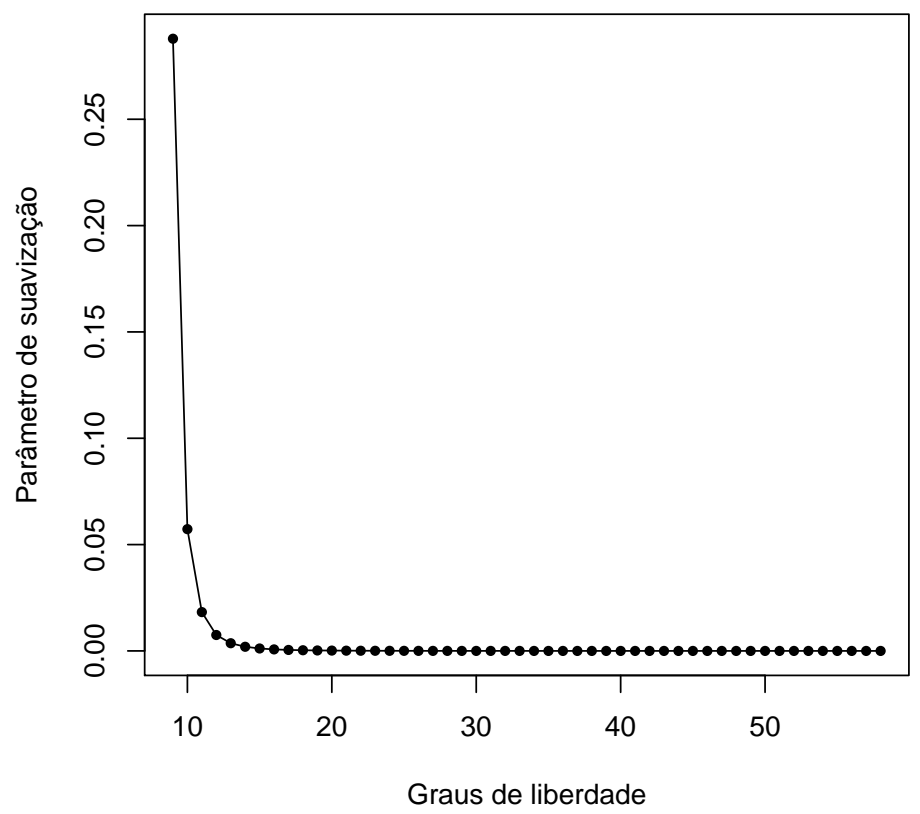

Figura 5.2: Valores do parâmetro de suavização variando os graus de liberdade.

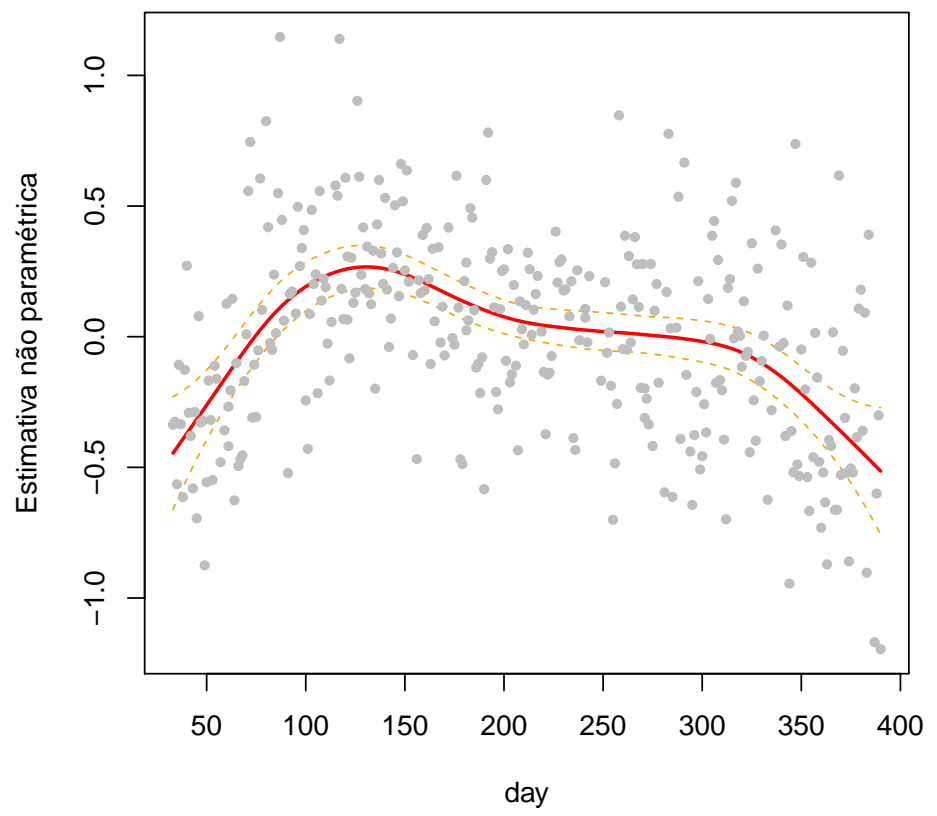

Figura 5.3: Função não paramétrica ajustada (spline cúbico) à variável explicativa day com a respectiva banda de confiança de $95 \%$. 
Tabela 5.1: Estimativas de máxima verossimilhança penalizadas com os respectivos erros padrão aproximados referentes ao modelo semiparamétrico com resposta binomial negativa ajustado aos dados de ozônio.

\begin{tabular}{crrr}
\hline Efeito & Estimativa & E. Padrão & valor $\mathbf{z}$ \\
\hline Intercepto & 0,974 & 0,014 & 6,853 \\
humidity & 0,006 & 0,001 & 4,526 \\
temp & 0,015 & 0,003 & 4,987 \\
ibh & $-5 \times 10^{-5}$ & $2 \times 10^{-5}$ & $-2,439$ \\
ibt & 0,003 & $8 \times 10^{-4}$ & 3,605 \\
vis & $-0,001$ & $3 \times 10^{-4}$ & $-3,331$ \\
$\log (\phi)$ & $-4,441$ & 0,571 & \\
$\hat{\lambda}$ & 0,003622 & & \\
$d f(\hat{\lambda})$ & 5,00094 & & \\
\hline
\end{tabular}

Na Figura 5.4 apresentamos uma adaptação do gráfico normal de probabilidades com bandas de confiança (envelope) gerado de $95 \%$ para o resíduo componente do desvio, referente ao modelo semiparamétrico com resposta binomial negativa descrito em (5.1) ajustado aos dados sobre ozônio. Nota-se que não há tendências sistemáticas nem pontos extremos. Todos os resíduos caem dentro do envelope gerado. Assim, não há evidências de que a resposta binomial negativa não seja adequada. Observe que o gráfico normal de probabilidades gerado é praticamente idêntico ao gráfico gerado para o modelo (1.2).

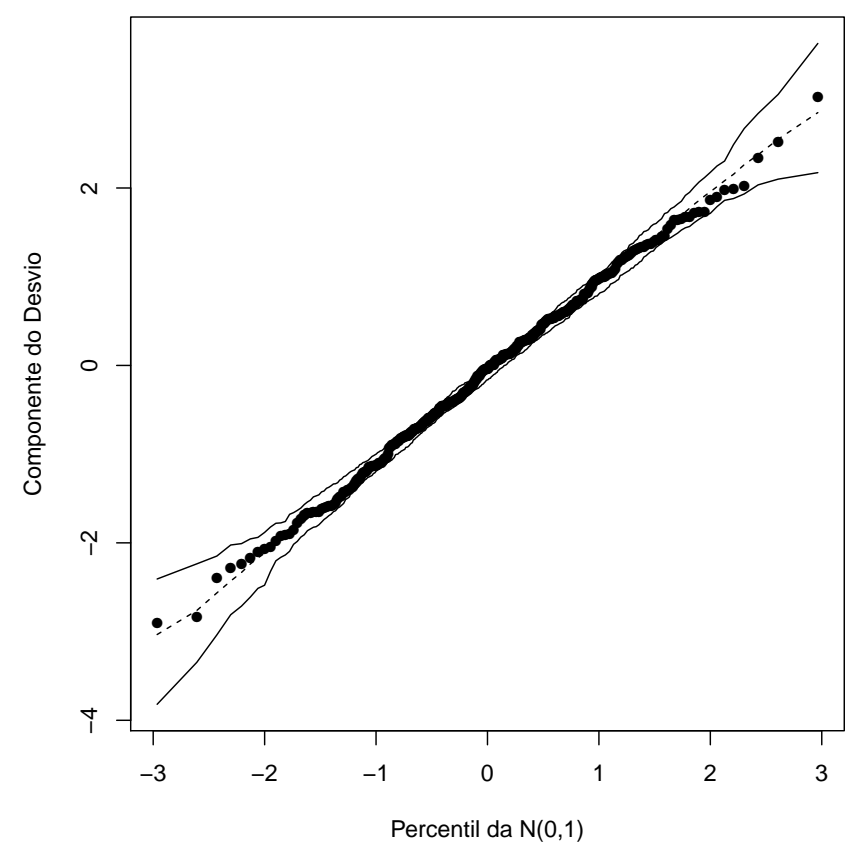

Figura 5.4: Gráfico normal de probabilidades com banda de confiança gerada de $95 \%$ para o resíduo componente do desvio referente ao modelo semiparamétrico com resposta binomial negativa ajustado aos dados de ozônio

Na Figura 5.5, apresentamos o gráfico gerado de índices $C_{i}(\boldsymbol{\theta})$ para o modelo (5.1) sob o 


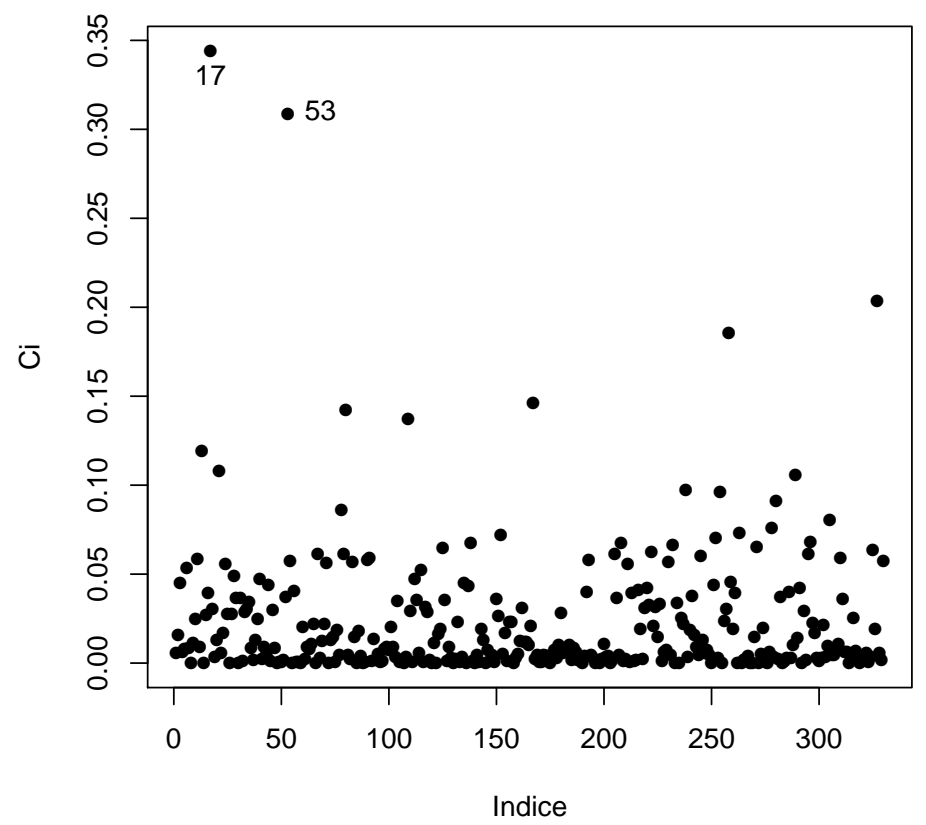

Figura 5.5: Gráfico de indices $C_{i}(\boldsymbol{\theta})$ sob o esquema de ponderação de casos.

trio de perturbação ponderação de casos e destaca alguns possíveis pontos influentes em $\boldsymbol{\theta}$. Para verificar se esses pontos são realmente de influência, retiramos um por vez e ajustamos novamente o modelo (5.1) verificando se há alguma mudança inferencial. Para este exemplo não houve mudança inferencial em nenhum caso estudado.

Com o intuito de avaliar a medida de influência local, com esse mesmo esquema de perturbação, para diferentes conjuntos de $\boldsymbol{\theta}$, construímos as Figuras 5.6, 5.7 e 5.8 representando a influência de cada observação para $\hat{\boldsymbol{\beta}}, \hat{\mathbf{f}}$ e $\hat{\phi}$ respectivamente.

Observe que em geral os índices mais elevados são os mesmos e como já analisado para os índices $C_{i}(\boldsymbol{\theta})$, as observações destacadas não interferem nos resultados inferenciais do modelo ajustado. Portanto, concluímos que o modelo semiparamétrico com resposta binomial negativa ajustou-se de forma bastante satisfatória aos dados de ozônio não havendo indícios de afastamentos das suposições feitas para o modelo nem presença de observações discrepantes com algum impacto importante no ajuste do modelo. 


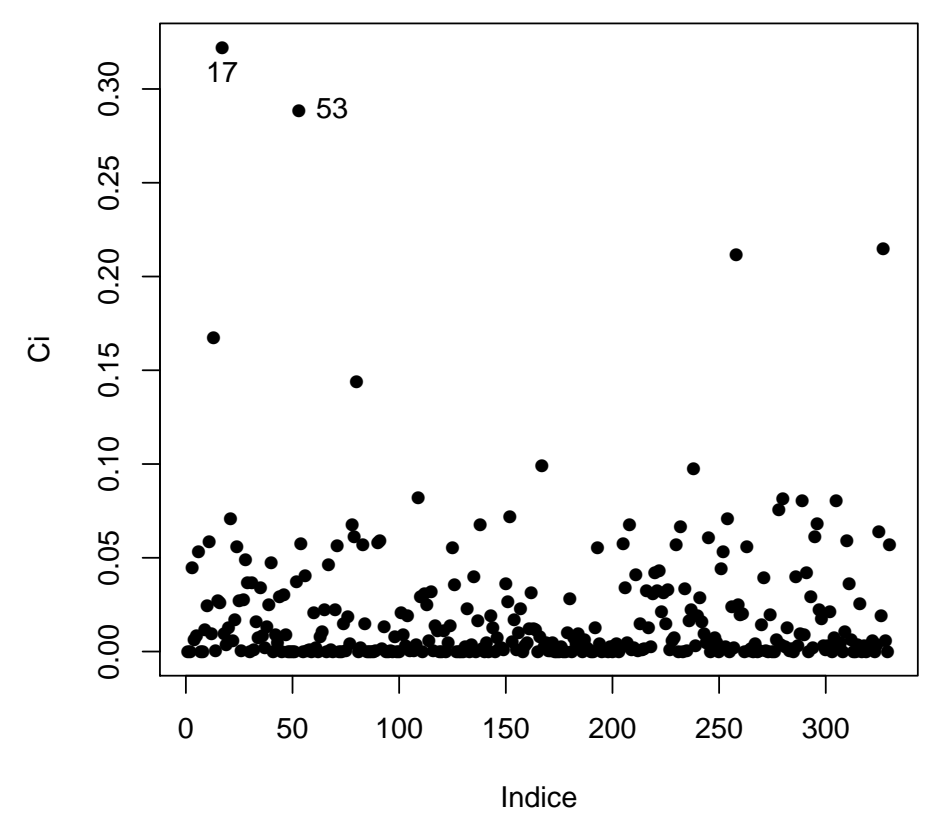

Figura 5.6: Gráfico de índices $C_{i}(\boldsymbol{\beta})$ sob o esquema de ponderação de casos.

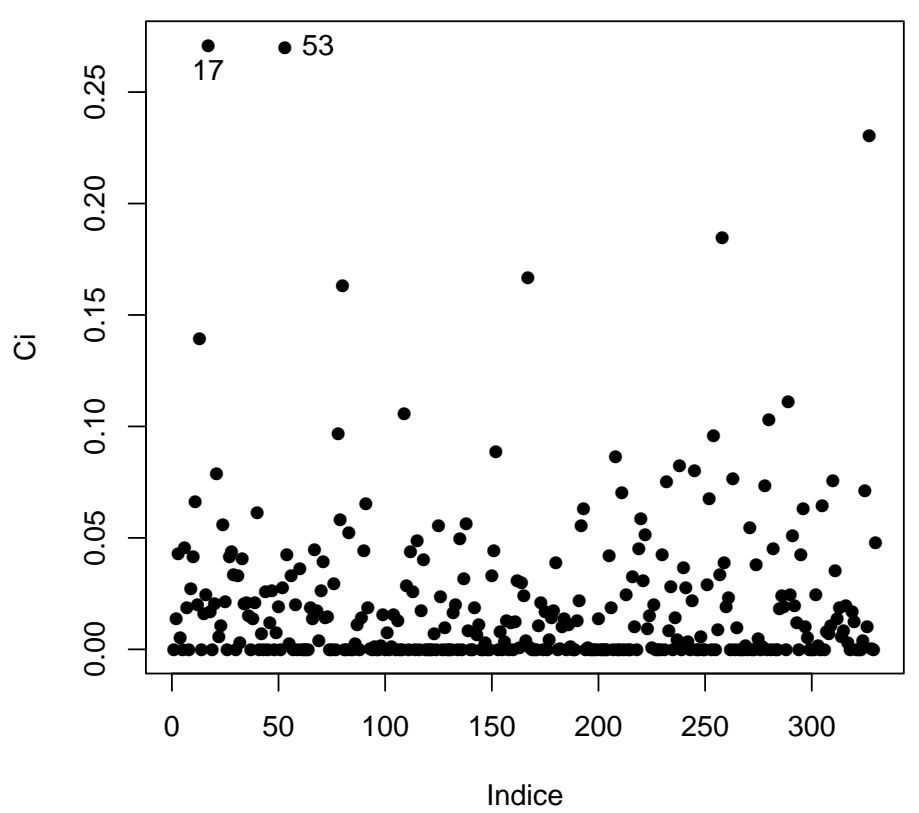

Figura 5.7: Gráfico de indices $C_{i}(\mathbf{f})$ sob o esquema de ponderação de casos. 


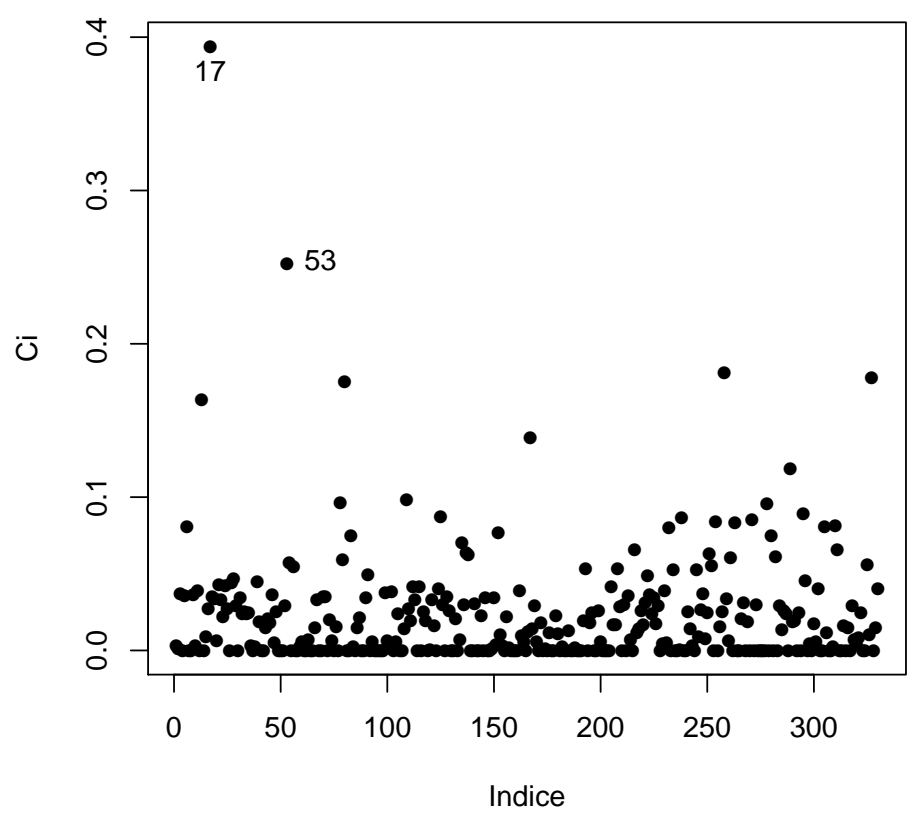

Figura 5.8: Gráfico de indices $C_{i}(\phi)$ sob o esquema de ponderação de casos. 


\section{Capítulo 6}

\section{Conclusões}

Neste trabalho apresentamos resultados de estimação e diagnóstico em modelos com resposta binomial negativa em que uma variável explicativa entra no modelo de forma não paramétrica. Foram utilizados splines cúbicos para o ajuste da função não paramétrica e foram aplicadas funções de verossimilhança penalizadas para a obtenção das estimativas de máxima verossimilhança penalizadas. Revisamos o procedimento iterativo para a estimação dos parâmetros, a estimação do parâmetro de suavização e dos graus de liberdade efetivos e apresentamos alguns resultados inferenciais.

Foram feitas também adaptações de alguns procedimentos de diagnóstico, tais como desenvolvimento de medidas de alavanca, adaptação da distância de Cook, análise de resíduos e influência local sob dois esquemas usuais de perturbação.

Ilustramos uma aplicação das metodologias com dados sobre concentração de ozônio descritas em Faraway (2006, Cap. 12) e verificamos uma excelente adequação do ajuste bem como superioridade com relação ao ajuste paramétrico, tanto do ponto de vista de metodologias de diagnóstico quanto adequação do ajuste através da medidas de informação.

Gráficos de diagnóstico foram adaptados para a aplicação e foram constatados pontos possivelmente influentes que quando retirados não alteraram do ponto de vista inferenciais os resultados.

\subsection{Considerações Finais}

Uma extensão natural do modelo é considerar mais de uma função não paramétrica no preditor linear, que é conhecido como modelos aditivos. Outra possível extensão seria a aplicação de $\mathrm{P}$-splines ao invés de splines cúbicos, reduzindo o custo computacional na estimação do componente não paramétrico. 


\section{Apêndice A}

\section{Gráfico da variável adicionada}

Vamos supor o modelo de regressão dado por

$$
y_{i}=\mathbf{x}_{i}^{T} \boldsymbol{\beta}+\gamma \omega_{i}+\epsilon_{i}
$$

$i=1, \ldots, n$, em que $\mathbf{x}_{i}=\left(x_{i 2}, \ldots, x_{i p}\right)^{T}$ contém valores de variáveis explicativas, $\omega_{i}$ é o valor de uma variável explicativa adicional qualquer e os erros $\epsilon_{i}$ 's são variáveis aleatórias independentes normalmente distribuídas de média zero e variância constante $\sigma^{2}$. Definindo $\mathbf{Z}=(\mathbf{X}, \boldsymbol{\omega})$, em que $\mathbf{X}=\left(\mathbf{x}_{1}^{T}, \ldots, \mathbf{x}_{n}^{T}\right)^{T}$ é a matriz modelo das variáveis explicativas, podemos mostrar facilmente que a estimativa de mínimos quadrados de $\boldsymbol{\theta}=\left(\boldsymbol{\beta}^{T}, \gamma\right)^{T}$ é dada por $\hat{\boldsymbol{\theta}}=\left(\mathbf{Z}^{T} \mathbf{Z}\right)^{-1} \mathbf{Z}^{T} \mathbf{y}$. Em particular obtemos, após alguma álgebra, que

$$
\hat{\gamma}=\frac{\boldsymbol{\omega}^{T}\left(\mathbf{I}_{n}-\mathbf{H}\right) \mathbf{y}}{\boldsymbol{\omega}^{T}\left(\mathbf{I}_{n}-\mathbf{H}\right) \boldsymbol{\omega}}=\frac{\boldsymbol{\omega} \mathbf{r}}{\boldsymbol{\omega}^{T}\left(\mathbf{I}_{n}-\mathbf{H}\right) \boldsymbol{\omega}}
$$

em que $\mathbf{I}_{n}$ é uma matriz identidade $n \times n$ e $\mathbf{H}$ é a matriz de projeção. $\hat{\gamma}$ é o coeficiente da regressão linear passando pela origem do vetor de resíduos $\mathbf{r}=\left(\mathbf{I}_{n}-\mathbf{H}\right) \mathbf{y}$ sobre o novo resíduo $\boldsymbol{v}=\left(\mathbf{I}_{n}-\mathbf{H}\right) \boldsymbol{\omega}$ dado por

$$
\begin{aligned}
\hat{\gamma} & =\left(\boldsymbol{v}^{T} \boldsymbol{v}\right)^{-1} \boldsymbol{v}^{T} \mathbf{r} \\
& =\left\{\boldsymbol{\omega}^{T}\left(\mathbf{I}_{n}-\mathbf{H}\right)\left(\mathbf{I}_{n}-\mathbf{H}\right) \boldsymbol{\omega}\right\}^{-1} \boldsymbol{\omega}^{T}\left(\mathbf{I}_{n}-\mathbf{H}\right)\left(\mathbf{I}_{n}-\mathbf{H}\right) \mathbf{y} \\
& =\frac{\boldsymbol{\omega}^{T}\left(\mathbf{I}_{n}-\mathbf{H}\right) \mathbf{y}}{\boldsymbol{\omega}^{T}\left(\mathbf{I}_{n}-\mathbf{H}\right) \boldsymbol{\omega}}
\end{aligned}
$$

Portanto, um gráfico de $\mathbf{r}$ contra $\boldsymbol{v}$ pode fornecer informações sobre a evidência dessa regressão, indicando quais observações que estão contribuindo para a relação e quais observações que estão se desviando da mesma. Esse gráfico, conhecido como gráfico da variável adicionada, pode revelar quais pontos que estão influenciando (e de que maneira) a inclusão da nova variável no modelo.

Analogamente vamos supor um modelo com resposta binomial negativa com $p$ parâmetros $\beta_{1}, \ldots, \beta_{p}, \phi$ conhecido, e que um parâmetro adicional $\gamma$ está sendo incluído no modelo. O 
interesse é testarmos $\mathrm{H}_{0}: \gamma=0$ contra $\mathrm{H}_{1}: \gamma \neq 0$.

Seja $\boldsymbol{\eta}(\boldsymbol{\beta}, \gamma)$ o preditor linear com $p+1$ parâmetros, isto é

$$
\boldsymbol{\eta}(\boldsymbol{\beta}, \gamma)=\mathbf{X} \boldsymbol{\beta}+\mathbf{Z} \gamma
$$

A função escore para $\gamma$ é dada por

$$
U_{\gamma}=\frac{\partial L(\boldsymbol{\beta}, \gamma)}{\partial \gamma}=\phi^{1 / 2} \mathbf{Z}^{T} \mathbf{W}^{1 / 2} \mathbf{r}_{p}
$$

em que $\mathbf{Z}=\left(z_{1}, \ldots, z_{n}\right)^{T}, \mathbf{W}$ a matriz de pesos e $\mathbf{r}_{p}$ é o vetor de resíduos.

Podemos mostrar, depois de algumas manipulações algébricas, que

$$
\operatorname{Var}(\hat{\gamma})=\phi^{-1}\left[\mathbf{Z}^{T} \mathbf{W}^{1 / 2} \mathbf{M} \mathbf{W}^{1 / 2} \mathbf{Z}\right]^{-1}
$$

em que $\mathbf{M}=\mathbf{I}_{n}-\mathbf{H}$.

Logo, $\operatorname{Var}(\hat{\gamma})=\phi^{-1}\left(\mathbf{R}^{T} \mathbf{W R}\right)^{-1} \operatorname{com} \mathbf{R}=\mathbf{Z}-\mathbf{X C}$ e $\mathbf{C}=\left(\mathbf{X}^{T} \mathbf{W X}\right)^{-1} \mathbf{X}^{T} \mathbf{W Z}$.

Assim, por exemplo, a estatística de escore para testar $\mathrm{H}_{0}: \gamma=0$ contra $\mathrm{H}_{1}: \gamma \neq 0$ fica dada por

$$
\xi_{S R}=\frac{\left(\hat{\mathbf{r}}_{p}^{T} \widehat{\mathbf{W}}^{1 / 2} \mathbf{Z}\right)^{2}}{\mathbf{Z}^{T} \widehat{\mathbf{W}}^{1 / 2} \widehat{\mathbf{M}} \widehat{\mathbf{W}}^{1 / 2} \mathbf{Z}}
$$

em que $\widehat{\mathbf{W}}, \hat{\mathbf{r}}_{p}$ e $\widehat{\mathbf{M}}$ são avaliados em $\hat{\boldsymbol{\beta}}\left(\right.$ sob $\left.\mathrm{H}_{0}\right)$. Sob $\mathrm{H}_{0}, \xi_{S R} \sim \chi_{1}^{2}$ quando $n \rightarrow \infty$.

Wang (1985) mostra que a estatística de escore acima coincide com a estatística $F$ de uma regressão linear ponderada para testar a inclusão da variável $\mathbf{Z}$ no modelo. Nessa regressão linear, o gráfico da variável adicionada é formado pelos resíduos $\hat{\mathbf{r}}_{p}$ e $\boldsymbol{v}=\phi^{1 / 2}\left(\mathbf{I}_{n}-\widehat{\mathbf{H}}\right) \widehat{\mathbf{W}}^{1 / 2} \mathbf{Z}$.

A principal dificuldade para construirmos o gráfico da variável adicionada em MLGs é a obtenção do resíduo $\boldsymbol{v}$, uma vez que o resíduo $\hat{\mathbf{r}}_{p}$ é obtido facilmente como já vimos anteriormente. O resíduo $\boldsymbol{v}$ pode ser obtido após a regressão linear ponderada (com pesos $\widehat{\mathbf{W}})$ de $\mathbf{Z}$ contra $\mathbf{X}$, assi temos que $\hat{\gamma}=\left(\boldsymbol{v}^{T} \boldsymbol{v}\right)^{-1} \boldsymbol{v}^{T} \mathbf{r}$.

Logo, o gráfico de $\hat{\mathbf{r}}_{p}$ contra $\boldsymbol{v}$ pode revelar quais observações estão contribuindo mais na significância de $\gamma$. 


\section{Apêndice B}

\section{Derivações do MBNS}

Neste apêndice são apresentados os detalhes dos cálculos relacionados às derivadas da função de verossimilhança penalizada, definida no Capítulo 3, necessários para obter as funções escore e informações de Fisher para o modelo semiparamétrico com resposta binomial negativa.Como visto na Seção 3.3.2, o logaritmo da função de verossimilhança penalizada do MSRBN (3.6) é dado por

$$
\begin{aligned}
\mathrm{L}_{p}(\boldsymbol{\theta}, \lambda) & =\sum_{i=1}^{n}\left[\log \left\{\frac{\Gamma\left(\phi+y_{i}\right)}{\Gamma\left(y_{i}+1\right) \Gamma(\phi)}\right\}+\phi \log \phi+y_{i} \log \mu_{i}-\left(\phi+y_{i}\right) \log \left(\mu_{i}+\phi\right)\right]- \\
& -\frac{\lambda}{2} \mathbf{f}^{T} \mathbf{K f},
\end{aligned}
$$

sendo $\boldsymbol{\theta}=\left(\boldsymbol{\beta}^{T}, \mathbf{f}^{T}, \phi\right)^{T}$ o vetor de parâmetros a serem estimados via maximização do logaritmo da função de verossimilhança penalizada. Para todos os cálculos realizados a seguir, estamos assumindo que (B.1) obedece às condições de regularidade e que todas as derivadas parciais com relação à $\boldsymbol{\beta}, \mathbf{f}$ e $\phi$ existem.

\section{B.1 Funções escore penalizadas}

Esta seção é dedicada a obtenção da função escore de cada parâmetro. Vamos apresentar detalhes dos cálculos relacionados à primeira derivada da função de verossimilhança penalizada. Calculando a derivada de (B.1) em relação a $\boldsymbol{\beta}$, temos o $j$-ésimo elemento da função 
escore penalizada dos efeitos paramétricos $\mathrm{U}_{p}^{\beta_{j}}$ dada por

$$
\begin{aligned}
\mathrm{U}_{p}^{\beta_{j}}(\boldsymbol{\theta})=\frac{\partial \mathrm{L}_{p}(\boldsymbol{\theta}, \lambda)}{\partial \beta_{j}} & =\sum_{i=1}^{n}\left\{\frac{y_{i}}{\mu_{i}} \frac{d \mu_{i}}{d \eta_{i}} \frac{\partial \eta_{i}}{\partial \beta_{j}}-\frac{\left(\phi+y_{i}\right)}{\left(\phi+\mu_{i}\right)} \frac{d \mu_{i}}{d \eta_{i}} \frac{\partial \eta_{i}}{\partial \beta_{j}}\right\} \\
& =\sum_{i=1}^{n}\left\{\frac{y_{i}}{\mu_{i}} \frac{d \mu_{i}}{d \eta_{i}} x_{i j}-\frac{\left(\phi+y_{i}\right)}{\left(\phi+\mu_{i}\right)} \frac{d \mu_{i}}{d \eta_{i}} x_{i j}\right\} \\
& =\sum_{i=1}^{n}\left\{\frac{\phi\left(d \mu_{i} / d \eta_{i}\right)}{\mu_{i}\left(\phi+\mu_{i}\right)}\left(y_{i}-\mu_{i}\right) x_{i j}\right\} \\
& =\sum_{i=1}^{n} w_{i} f_{i}^{*-1}\left(y_{i}-\mu_{i}\right) x_{i j},
\end{aligned}
$$

em que $j=1, \ldots, p, w_{i}=\left(d \mu_{i} / d \eta_{i}\right)^{2} /\left(\mu_{i}^{2} \phi^{-1}+\mu_{i}\right), f_{i}^{*}=d \mu_{i} / d \eta_{i}$ e $\mu_{i}=g^{-1}\left(\mathbf{x}_{i}^{T} \boldsymbol{\beta}+\mathbf{n}_{i}^{T} \mathbf{f}\right) \operatorname{com}$ $g(\cdot)$ sendo a função de ligação. No Capítulo 2 são apresentadas as quantidades $w_{i}$ e $f_{i}^{*}$ para diferentes funções de ligação.

Em relação ao vetor $(h \times 1)$ de efeito não paramétrico $\mathbf{f}$, temos a função escore penalizada $\mathrm{U}_{p}^{f_{j}}$ dada por

$$
\begin{aligned}
\mathrm{U}_{p}^{f_{j}}(\boldsymbol{\theta})=\frac{\partial \mathrm{L}_{p}(\boldsymbol{\theta}, \lambda)}{\partial f_{j}} & =\sum_{i=1}^{n}\left\{\frac{y_{i}}{\mu_{i}} \frac{d \mu_{i}}{d \eta_{i}} \frac{\partial \eta_{i}}{\partial f_{j}}-\frac{\left(\phi+y_{i}\right)}{\left(\phi+\mu_{i}\right)} \frac{d \mu_{i}}{d \eta_{i}} \frac{\partial \eta_{i}}{\partial f_{j}}\right\}-[\lambda \mathbf{K f}]_{j} \\
& =\sum_{i=1}^{n}\left\{\frac{y_{i}}{\mu_{i}} \frac{d \mu_{i}}{d \eta_{i}} n_{i j}-\frac{\left(\phi+y_{i}\right)}{\left(\phi+\mu_{i}\right)} \frac{d \mu_{i}}{d \eta_{i}} n_{i j}\right\}-[\lambda \mathbf{K f}]_{j} \\
& =\sum_{i=1}^{n}\left\{\frac{\phi\left(d \mu_{i} / d \eta_{i}\right)}{\mu_{i}\left(\phi+\mu_{i}\right)}\left(y_{i}-\mu_{i}\right) n_{i j}\right\}-[\lambda \mathbf{K f}]_{j} \\
& =\sum_{i=1}^{n} w_{i} f_{i}^{*-1}\left(y_{i}-\mu_{i}\right) n_{i j}-[\lambda \mathbf{K f}]_{j},
\end{aligned}
$$

sendo $a_{j}$ o $j$-ésimo elemento do vetor $\lambda \mathbf{K f} \operatorname{com} j=1, \ldots, h$.

Finalmente, para o parâmetro de dispersão $\phi$, temos a função escore penalizada $\mathrm{U}_{p}^{\phi}(\boldsymbol{\theta})$ dada por

$$
\mathrm{U}_{p}^{\phi}(\boldsymbol{\theta})=\frac{\partial \mathrm{L}_{p}(\boldsymbol{\theta}, \lambda)}{\partial \phi}=\sum_{i=1}^{n}\left[\psi\left(\phi+y_{i}\right)-\psi(\phi)-\frac{\left(y_{i}+\phi\right)}{\left(\phi+\mu_{i}\right)}+\log \left\{\frac{\phi}{\left(\phi+\mu_{i}\right)}\right\}+1\right]
$$

em que a função $\psi(\cdot)$ é chamada função digama.

Para facilitar o manuseio computacional destas funções, podemos escrever (B.2) e (B.3) na forma matricial

$$
\begin{aligned}
\mathbf{U}_{p}^{\boldsymbol{\beta}}(\boldsymbol{\theta}) & =\mathbf{X}^{T} \mathbf{W} \mathbf{F}^{-1}(\mathbf{y}-\boldsymbol{\mu}) \mathrm{e} \\
\mathbf{U}_{p}^{\mathbf{f}}(\boldsymbol{\theta}) & =\mathbf{N}^{T} \mathbf{W} \mathbf{F}^{-1}(\mathbf{y}-\boldsymbol{\mu})-\lambda \mathbf{K f} .
\end{aligned}
$$




\section{B.2 Informação de Fisher penalizadas}

Apresentamos nesta seção os cálculos para a obtenção da matriz observada de Fisher (matriz Hessiana), em que basta tomar a segunda derivada do vetor escore em relação a $\boldsymbol{\theta}$. Mostramos também a matriz de informação de Fisher.

Calculando novamente a derivada de (B.2) em relação ao vetor $\boldsymbol{\beta}$, temos a segunda derivada do logaritmo da função de verossimilhança penalizada dada por

$$
\begin{aligned}
\ddot{\mathrm{L}}_{p}^{\beta_{j} \beta_{l}} & =\frac{\partial^{2} \mathrm{~L}_{p}(\boldsymbol{\theta}, \lambda)}{\partial \beta_{j} \partial \beta_{l}}=\frac{\partial \mathrm{U}_{p}^{\beta_{j}}(\boldsymbol{\theta})}{\partial \beta_{l}} \\
& =\frac{\partial}{\partial \beta_{l}} \sum_{i=1}^{n} \frac{f_{i}^{*}}{\left(\mu_{i}^{2} \phi^{-1}+\mu_{i}\right)}\left(y_{i}-\mu_{i}\right) x_{i j} \\
& =\sum_{i=1}^{n} f_{i}^{*} x_{i j} \underbrace{\frac{\partial}{\partial \beta_{l}} \frac{\left(y_{i}-\mu_{i}\right)}{\left(\mu_{i}^{2} \phi^{-1}+\mu_{i}\right)}}_{(\mathbf{I})} .
\end{aligned}
$$

Calculando (I) pela regra da cadeia obtemos

$$
\begin{aligned}
\frac{\partial}{\partial \beta_{l}} \frac{\left(y_{i}-\mu_{i}\right)}{\left(\mu_{i}^{2} \phi^{-1}+\mu_{i}\right)} & =\frac{-\left(d \mu_{i} / d \eta_{i}\right) x_{i l}\left(\mu_{i}^{2} \phi^{-1}+\mu_{i}\right)-\left(y_{i}-\mu_{i}\right)\left(d \mu_{i} / d \eta_{i}\right) x_{i l}\left(2 \phi^{-1} \mu_{i}+1\right)}{\left(\mu_{i}^{2} \phi^{-1}+\mu_{i}\right)^{2}} \\
& =\frac{-f_{i}^{*} x_{i l}}{\left(\mu_{i}^{2} \phi^{-1}+\mu_{i}\right)}-\frac{\left(y_{i}-\mu_{i}\right) f_{i}^{*} x_{i l}\left(2 \phi^{-1} \mu_{i}+1\right)}{\left(\mu_{i}^{2} \phi^{-1}+\mu_{i}\right)^{2}},
\end{aligned}
$$

$\log 0$

$$
\ddot{\mathrm{L}}_{p}^{\beta_{j} \beta_{l}}=-\sum_{i=1}^{n} \frac{f_{i}^{* 2} x_{i j} x_{i l}}{\left(\mu_{i}^{2} \phi^{-1}+\mu_{i}\right)}-\sum_{i=1}^{n} \frac{f_{i}^{* 2} x_{i j} x_{i l}\left(y_{i}-\mu_{i}\right)\left(2 \phi^{-1} \mu_{i}+1\right)}{\left(\mu_{i}^{2} \phi^{-1}+\mu_{i}\right)^{2}} .
$$

Assim, em forma matricial, obtemos

$$
\ddot{\mathrm{L}_{p}^{\beta} \boldsymbol{\beta}}=\frac{\partial^{2} \mathrm{~L}_{p}(\boldsymbol{\theta}, \lambda)}{\partial \boldsymbol{\beta} \partial \boldsymbol{\beta}^{T}}=-\mathbf{X}^{T} \mathbf{W X}-\mathbf{X}^{T} \mathbf{A}(\mathbf{a}) \mathbf{X}(\mathbf{y}-\boldsymbol{\mu}),
$$

em que $\mathbf{A}(\mathbf{a})=\operatorname{diag}\left\{a_{1}, \ldots, a_{n}\right\}, \operatorname{com} a_{i}=f_{i}^{* 2} \frac{\left(2 \phi^{-1} \mu_{i}+1\right)}{\left(\mu_{i}^{2} \phi^{-1}+\mu_{i}\right)^{2}}$, para $i=1, \ldots, n$.

Para o vetor de parâmetros $\mathbf{f}$, os cálculos são similares aos feitos para $\boldsymbol{\beta}$, modificando-se 
com a inclusão do termo referente à matriz de penalização, logo,

$$
\begin{aligned}
\ddot{\mathrm{L}}_{p}^{f_{j} f_{l}} & =\frac{\partial^{2} \mathrm{~L}_{p}(\boldsymbol{\theta}, \lambda)}{\partial f_{j} \partial f_{l}}=\frac{\partial \mathrm{U}_{p}^{f_{j}}(\boldsymbol{\theta})}{\partial f_{l}} \\
& =\frac{\partial}{\partial f_{l}} \sum_{i=1}^{n} \frac{f_{i}^{*}}{\left(\mu_{i}^{2} \phi^{-1}+\mu_{i}\right)}\left(y_{i}-\mu_{i}\right) n_{i j}-\frac{\partial}{\partial f_{l}}[\lambda \mathbf{K} \mathbf{f}]_{j} \\
& =\sum_{i=1}^{n} f_{i}^{*} n_{i j} \frac{\partial}{\partial f_{l}} \frac{\left(y_{i}-\mu_{i}\right)}{\left(\mu_{i}^{2} \phi^{-1}+\mu_{i}\right)}-\lambda k_{j l} \\
& =-\sum_{i=1}^{n} \frac{f_{i}^{* 2} n_{i j} n_{i l}}{\left(\mu_{i}^{2} \phi^{-1}+\mu_{i}\right)}-\sum_{i=1}^{n} \frac{f_{i}^{* 2} n_{i j} n_{i l}\left(y_{i}-\mu_{i}\right)\left(2 \phi^{-1} \mu_{i}+1\right)}{\left(\mu_{i}^{2} \phi^{-1}+\mu_{i}\right)^{2}}-\lambda k_{j l} .
\end{aligned}
$$

em que $k_{j l}$ é o $(j l)$-ésimo elemento de $\mathbf{K}$. Assim, de forma matricial obtemos

$$
\ddot{\mathrm{L}}_{p}=\frac{\partial^{2} \mathrm{~L}_{p}(\boldsymbol{\theta}, \lambda)}{\partial \mathbf{f} \partial \mathbf{f}^{T}}=-\mathbf{N}^{T} \mathbf{W} \mathbf{N}-\mathbf{N}^{T} \mathbf{A}(\mathbf{a}) \mathbf{N}(\mathbf{y}-\boldsymbol{\mu})-\lambda \mathbf{K} .
$$

Em relação a $\phi$, encontramos

$$
\begin{aligned}
\ddot{\mathrm{L}}_{p}^{\phi \phi} & =\frac{\partial^{2} \mathrm{~L}_{p}(\boldsymbol{\theta}, \lambda)}{\partial \phi \partial \phi}=\frac{\partial \mathrm{U}_{p}^{\phi}(\boldsymbol{\theta})}{\partial \phi} \\
& =\sum_{i=1}^{n}\left[\frac{\partial}{\partial \phi} \psi\left(\phi+y_{i}\right)-\frac{\partial}{\partial \phi} \psi(\phi)-\frac{\partial}{\partial \phi} \frac{\left(y_{i}+\phi\right)}{\left(\phi+\mu_{i}\right)}+\frac{\partial}{\partial \phi} \log \{\phi\}-\frac{\partial}{\partial \phi} \log \left\{\phi+\mu_{i}\right\}\right] \\
& =\sum_{i=1}^{n}\left[\psi^{\prime}\left(\phi+y_{i}\right)-\psi^{\prime}(\phi)+\frac{\left(y_{i}-\mu_{i}\right)}{\left(\phi+\mu_{i}\right)^{2}}+\frac{1}{\phi}-\frac{1}{\phi+\mu_{i}}\right] \\
& =\sum_{i=1}^{n}\left\{\psi^{\prime}\left(\phi+y_{i}\right)+\frac{\left(y_{i}-2 \mu_{i}-\phi\right)}{\left(\phi+\mu_{i}\right)^{2}}\right\}+\frac{n}{\phi}\left[1-\phi \psi^{\prime}(\phi)\right],
\end{aligned}
$$

em que a função $\psi^{\prime}(\cdot)$ é a função trigama.

Os termos mostrados anteriormente são referentes à diagonal principal da matriz $\ddot{\mathrm{L}}_{p}^{\boldsymbol{\theta} \boldsymbol{\theta}}$. Agora, prosseguimos para o cálculo das submatrizes cruzadas. Primeiramente mostramos os elementos de $\boldsymbol{\beta}$ em relação à f. Temos que

$$
\begin{aligned}
\ddot{\mathrm{L}}_{p}^{\beta_{j} \mathbf{f}_{l}} & =\frac{\partial^{2} \mathrm{~L}_{p}(\boldsymbol{\theta}, \lambda)}{\partial \beta_{j} \partial \mathbf{f}_{l}}=\frac{\partial \mathrm{U}_{p}^{\beta_{j}}(\boldsymbol{\theta})}{\partial \mathbf{f}_{l}} \\
& =\frac{\partial}{\partial \mathbf{f}_{l}} \sum_{i=1}^{n} \frac{f_{i}^{*}}{\left(\mu_{i}^{2} \phi^{-1}+\mu_{i}\right)}\left(y_{i}-\mu_{i}\right) x_{i j} \\
& =\sum_{i=1}^{n} f_{i}^{*} x_{i j} \underbrace{\frac{\partial}{\partial \mathbf{f}_{l}} \frac{\left(y_{i}-\mu_{i}\right)}{\left(\mu_{i}^{2} \phi^{-1}+\mu_{i}\right)}}_{(\mathbf{I I})} .
\end{aligned}
$$


Calculando (II) pela regra da cadeia obtemos

$$
\begin{aligned}
\frac{\partial}{\partial \mathbf{f}_{l}} \frac{\left(y_{i}-\mu_{i}\right)}{\left(\mu_{i}^{2} \phi^{-1}+\mu_{i}\right)} & =\frac{-\left(d \mu_{i} / d \eta_{i}\right) n_{i l}\left(\mu_{i}^{2} \phi^{-1}+\mu_{i}\right)-\left(y_{i}-\mu_{i}\right)\left(d \mu_{i} / d \eta_{i}\right) n_{i l}\left(2 \phi^{-1} \mu_{i}+1\right)}{\left(\mu_{i}^{2} \phi^{-1}+\mu_{i}\right)^{2}} \\
& =\frac{-f_{i}^{*} n_{i l}}{\left(\mu_{i}^{2} \phi^{-1}+\mu_{i}\right)}-\frac{\left(y_{i}-\mu_{i}\right) f_{i}^{*} n_{i l}\left(2 \phi^{-1} \mu_{i}+1\right)}{\left(\mu_{i}^{2} \phi^{-1}+\mu_{i}\right)^{2}},
\end{aligned}
$$

$\log 0$

$$
\ddot{\mathrm{L}}_{p}^{\beta_{j} \mathbf{f}_{l}}=-\sum_{i=1}^{n} \frac{f_{i}^{* 2} x_{i j} n_{i l}}{\left(\mu_{i}^{2} \phi^{-1}+\mu_{i}\right)}-\sum_{i=1}^{n} \frac{f_{i}^{* 2} x_{i j} n_{i l}\left(y_{i}-\mu_{i}\right)\left(2 \phi^{-1} \mu_{i}+1\right)}{\left(\mu_{i}^{2} \phi^{-1}+\mu_{i}\right)^{2}} .
$$

Assim, em forma matricial, obtemos

$$
\ddot{\mathrm{L}}_{p}^{\beta \boldsymbol{f}}=\frac{\partial^{2} \mathrm{~L}_{p}(\boldsymbol{\theta}, \lambda)}{\partial \boldsymbol{\beta} \partial \mathbf{f}^{T}}=-\mathbf{X}^{T} \mathbf{W N}-\mathbf{X}^{T} \mathbf{A}(\mathbf{a}) \mathbf{N}(\mathbf{y}-\boldsymbol{\mu}) .
$$

Já, para os termo cruzado entre $\boldsymbol{\beta}$ e $\phi$, obtemos

$$
\begin{aligned}
\ddot{\mathrm{L}}_{p}^{\beta_{j} \phi} & =\frac{\partial^{2} \mathrm{~L}_{p}(\boldsymbol{\theta}, \lambda)}{\partial \beta_{j} \partial \phi}=\frac{\partial \mathrm{U}_{p}^{\beta_{j}}(\boldsymbol{\theta})}{\partial \phi} \\
& =\frac{\partial}{\partial \phi} \sum_{i=1}^{n} \frac{f_{i}^{*}}{\left(\mu_{i}^{2} \phi^{-1}+\mu_{i}\right)}\left(y_{i}-\mu_{i}\right) x_{i j} \\
& =\sum_{i=1}^{n} f_{i}^{*}\left(y_{i}-\mu_{i}\right) x_{i j} \frac{\partial}{\partial \phi} \frac{1}{\left(\mu_{i}^{2} \phi^{-1}+\mu_{i}\right)} \\
& =\sum_{i=1}^{n} f_{i}^{*}\left(y_{i}-\mu_{i}\right) x_{i j} \frac{\mu_{i}^{2} \phi^{-2}}{\left(\mu_{i}^{2} \phi^{-1}+\mu_{i}\right)^{2}} \\
& =\sum_{i=1}^{n} \frac{f_{i}^{*} \phi^{-2}}{\left(\mu_{i} \phi^{-1}+1\right)^{2}}\left(y_{i}-\mu_{i}\right) x_{i j} .
\end{aligned}
$$

Logo, em forma matricial, obtemos

$$
\ddot{\mathrm{L}}_{p}^{\boldsymbol{\beta} \phi}=\frac{\partial^{2} \mathrm{~L}_{p}(\boldsymbol{\theta}, \lambda)}{\partial \boldsymbol{\beta} \partial \phi^{T}}=\mathbf{X}^{T} \mathbf{A}(\mathbf{b})(\mathbf{y}-\boldsymbol{\mu}),
$$

em que $\mathbf{A}(\mathbf{b})=\operatorname{diag}\left\{b_{1}, \ldots, b_{n}\right\}, \operatorname{com} b_{i}=\frac{f_{i}^{*} \phi^{-2}}{\left(\mu_{i} \phi^{-1}+1\right)^{2}}$, para $i=1, \ldots, n$.

Os cálculos dos elementos cruzados do parâmetro f em relação a $\phi$ são similares aos 
mostrados acima. Assim, obtemos

$$
\begin{aligned}
\ddot{\mathrm{L}}_{p}^{\mathbf{f}_{j} \phi} & =\frac{\partial^{2} \mathrm{~L}_{p}(\boldsymbol{\theta}, \lambda)}{\partial \mathbf{f}_{j} \partial \phi}=\frac{\partial \mathrm{U}_{p}^{\mathbf{f}_{j}}(\boldsymbol{\theta})}{\partial \phi} \\
& =\frac{\partial}{\partial \phi} \sum_{i=1}^{n} \frac{f_{i}^{*}}{\left(\mu_{i}^{2} \phi^{-1}+\mu_{i}\right)}\left(y_{i}-\mu_{i}\right) n_{i j} \\
& =\sum_{i=1}^{n} f_{i}^{*}\left(y_{i}-\mu_{i}\right) n_{i j} \frac{\partial}{\partial \phi} \frac{1}{\left(\mu_{i}^{2} \phi^{-1}+\mu_{i}\right)} \\
& =\sum_{i=1}^{n} \frac{f_{i}^{*} \phi^{-2}}{\left(\mu_{i} \phi^{-1}+1\right)^{2}}\left(y_{i}-\mu_{i}\right) n_{i j} .
\end{aligned}
$$

Logo, em forma matricial, obtemos

$$
\ddot{\mathrm{L}}{ }_{p}^{\mathbf{f} \phi}=\frac{\partial^{2} \mathrm{~L}_{p}(\boldsymbol{\theta}, \lambda)}{\partial \mathbf{f} \partial \phi^{T}}=\mathbf{N}^{T} \mathbf{A}(\mathbf{b})(\mathbf{y}-\boldsymbol{\mu}) .
$$

Para os cálculos dos elementos da matriz de Fisher, apresentados nesta seção, inicialmente lembramos que $\mathrm{E}(\mathbf{y})=\boldsymbol{\mu}$, em que $Y_{i} \sim \mathrm{BN}\left(\mu_{i}, \phi\right)$. Assim as submatrizes bloco diagonal da matriz de informação de Fisher ficam dadas por

$$
\begin{aligned}
\mathbf{K}_{p}^{\boldsymbol{\beta} \boldsymbol{\beta}} & =-\mathrm{E}\left(\ddot{\mathrm{L}}_{p}^{\boldsymbol{\beta} \boldsymbol{\beta}}\right)=-\mathrm{E}\left(-\mathbf{X}^{T} \mathbf{W X}-\mathbf{X}^{T} \mathbf{A}(\mathbf{a}) \mathbf{X}(\mathbf{y}-\boldsymbol{\mu})\right) \\
& =\mathbf{X}^{T} \mathbf{W} \mathbf{X}+\left[\mathbf{X}^{T} \mathbf{A}(\mathbf{a}) \mathbf{X}\right] \mathrm{E}(\mathbf{y}-\boldsymbol{\mu}) \\
& =\mathbf{X}^{T} \mathbf{W} \mathbf{X} \\
\mathbf{K}_{p}^{\mathrm{ff}}= & -\mathrm{E}\left(\ddot{\mathrm{L}}{ }_{p}\right)=-\mathrm{E}\left(-\mathbf{N}^{T} \mathbf{W} \mathbf{N}-\mathbf{N}^{T} \mathbf{A}(\mathbf{a}) \mathbf{N}(\mathbf{y}-\boldsymbol{\mu})-\lambda \mathbf{K}\right) \\
= & \mathbf{N}^{T} \mathbf{W N}-\lambda \mathbf{K}+\left[\mathbf{N}^{T} \mathbf{A}(\mathbf{a}) \mathbf{N}\right] \mathrm{E}(\mathbf{y}-\boldsymbol{\mu}) \\
= & \mathbf{N}^{T} \mathbf{W N}-\lambda \mathbf{K}
\end{aligned}
$$

e a informação de Fisher para $\phi$, que pode ser verificada em Lawless (1987), fica dada por

$$
\mathrm{K}_{p}^{\phi \phi}=\sum_{i=1}^{n}\left\{\sum_{j=0}^{\infty} \frac{\mathrm{P}_{r}\left(Y_{i} \geq j\right)}{(\phi+j)^{2}}-\frac{\mu_{i}}{\phi\left(\mu_{i}+\phi\right)}\right\} .
$$

Já as submatrizes bloco cruzadas são dadas por

$$
\begin{aligned}
\mathbf{K}_{p}^{\boldsymbol{\beta} \mathbf{f}} & =-\mathrm{E}\left(\ddot{\mathrm{L}}_{p}^{\boldsymbol{\beta} \boldsymbol{\beta}}\right)=-\mathrm{E}\left(-\mathbf{X}^{T} \mathbf{W} \mathbf{X}-\mathbf{X}^{T} \mathbf{A}(\mathbf{a}) \mathbf{X}(\mathbf{y}-\boldsymbol{\mu})\right) \\
& =\mathbf{X}^{T} \mathbf{W} \mathbf{X}+\left[\mathbf{X}^{T} \mathbf{A}(\mathbf{a}) \mathbf{X}\right] \mathrm{E}(\mathbf{y}-\boldsymbol{\mu}) \\
& =\mathbf{X}^{T} \mathbf{W X},
\end{aligned}
$$




$$
\begin{aligned}
\mathbf{K}_{p}^{\boldsymbol{\beta} \phi} & =-\mathrm{E}\left(\ddot{\mathrm{L}}_{p}^{\boldsymbol{\beta} \phi}\right)=-\mathrm{E}\left(\mathbf{X}^{T} \mathbf{A}(\mathbf{b})(\mathbf{y}-\boldsymbol{\mu})\right) \\
& =-\mathbf{X}^{T} \mathbf{A}(\mathbf{b}) \mathrm{E}(\mathbf{y}-\boldsymbol{\mu}) \\
& =\mathbf{0} .
\end{aligned}
$$

e

$$
\begin{aligned}
\mathbf{K}_{p}^{\mathbf{f} \phi} & =-\mathrm{E}\left(\ddot{\mathrm{L}}_{p}^{\mathbf{f} \boldsymbol{\beta}}\right)=-\mathrm{E}\left(\mathbf{N}^{T} \mathbf{A}(\mathbf{b})(\mathbf{y}-\boldsymbol{\mu})\right) \\
& =-\mathbf{N}^{T} \mathbf{A}(\mathbf{b}) \mathrm{E}(\mathbf{y}-\boldsymbol{\mu}) \\
& =\mathbf{0}
\end{aligned}
$$

Note por estes resultados que os vetores $\boldsymbol{\beta}$ e $\mathbf{f}$ são ortogonais a $\phi$. 


\section{Apêndice C}

\section{Diagnóstico}

Nesta seção apresentamos os cálculos da matriz $\boldsymbol{\Delta}$, definida no Capítulo 4 e utilizada para detectar possíveis mudanças importantes nas estimativas dos parâmetros sob pequenas perturbações no modelo ou nos dados. Encontramos tal matriz para dois esquemas de perturbação que serão apresentados em seções separadas. Primeiramente, avaliamos a contribuição individual de cada observação, considerando que cada observação possui um peso diferente no processo de estimação. Este esquema se chama ponderação de casos e é um dos mais utilizados na prática. Já no segundo esquema, chamado perturbação aditiva em uma variável contínua do modelo, supomos que uma das variáveis explicativas (contínua) seja acrescida de um delta.

\section{C.1 Ponderação de casos}

Consideramos que cada observação possui um peso diferente no processo de estimação, assim, iremos supor que o logaritmo da verossimilhança seja dado por

$$
\mathrm{L}_{p}(\boldsymbol{\theta}, \lambda \mid \boldsymbol{\delta})=\sum_{i=1}^{n} \delta_{i} \mathrm{~L}_{i}(\boldsymbol{\theta})-\frac{\lambda}{2} \mathbf{f}^{T} \mathbf{K f}
$$

em que

$$
\mathrm{L}_{i}(\boldsymbol{\theta})=\log \left\{\frac{\Gamma\left(\phi+y_{i}\right)}{\Gamma\left(y_{i}+1\right) \Gamma(\phi)}\right\}+\phi \log \phi+y_{i} \log \mu_{i}-\left(\phi+y_{i}\right) \log \left(\mu_{i}+\phi\right),
$$

$\boldsymbol{\delta}=\left(\delta_{1}, \ldots, \delta_{n}\right)^{T}$ é o vetor de pesos, com $0 \leq \delta_{i} \leq 1$. Neste esquema de perturbação, o vetor de não perturbação é dado por $\boldsymbol{\delta}_{0}=(1, \ldots, 1)^{T}$.

Podemos observar que, para este esquema de perturbação, as matrizes $\boldsymbol{\Delta}_{1 j i}, \boldsymbol{\Delta}_{2 j i}$ e $\boldsymbol{\Delta}_{3 i}$ são obtidas da seguinte forma:

$$
\begin{aligned}
\boldsymbol{\Delta}_{1 j i} & =\frac{\partial^{2} \mathrm{~L}_{p}(\boldsymbol{\theta}, \lambda \mid \boldsymbol{\delta})}{\partial \beta_{j} \partial \delta_{i}} \\
& =\frac{\partial}{\partial \delta_{i}} \delta_{i} w_{i} f_{i}^{*-1}\left(y_{i}-\mu_{i}\right) x_{i j} \\
& =w_{i} f_{i}^{*-1}\left(y_{i}-\mu_{i}\right) x_{i j}
\end{aligned}
$$

com forma matricial dada por

$$
\boldsymbol{\Delta}_{1}=\mathbf{X}^{T} \mathbf{D}(\mathbf{v}),
$$


em que $\mathbf{D}(v)$ é uma matriz diagonal $(n \times n)$ com elementos, dados por $\mathbf{v}=\left(v_{1}, \ldots, v_{n}\right)^{T}$ $\operatorname{com} v_{i}=\left(w_{i} / f_{i}\right)\left(y_{i}-\mu_{i}\right)$.

Analogamente, temos

$$
\begin{aligned}
\boldsymbol{\Delta}_{2 j i} & =\frac{\partial^{2} \mathrm{~L}_{p}(\boldsymbol{\theta}, \lambda \mid \boldsymbol{\delta})}{\partial f_{j} \partial \delta_{i}} \\
& =\frac{\partial}{\partial \delta_{i}} \delta_{i} w_{i} f_{i}^{*-1}\left(y_{i}-\mu_{i}\right) n_{i j}-\frac{\partial}{\partial \delta_{i}}[\lambda \mathbf{K f}]_{j} \\
& =w_{i} f_{i}^{*-1}\left(y_{i}-\mu_{i}\right) n_{i j} .
\end{aligned}
$$

com forma matricial dada por

$$
\Delta_{2}=\mathbf{N}^{T} \mathbf{D}(\mathbf{v})
$$

Por fim temos

$$
\begin{aligned}
\boldsymbol{\Delta}_{3 i} & =\frac{\partial^{2} \mathrm{~L}_{p}(\boldsymbol{\theta}, \lambda \mid \boldsymbol{\delta})}{\partial \phi \partial \delta_{i}} \\
& =\frac{\partial}{\partial \delta_{i}} \delta_{i}\left[\psi\left(\phi+y_{i}\right)-\psi(\phi)-\frac{\left(y_{i}+\phi\right)}{\left(\phi+\mu_{i}\right)}+\log \left\{\frac{\phi}{\left(\phi+\mu_{i}\right)}\right\}+1\right] \\
& =\psi\left(\phi+y_{i}\right)-\psi(\phi)-\frac{\left(y_{i}+\phi\right)}{\left(\phi+\mu_{i}\right)}+\log \left\{\frac{\phi}{\left(\phi+\mu_{i}\right)}\right\}+1
\end{aligned}
$$

com forma matricial dada por

$$
\boldsymbol{\Delta}_{3}=\mathbf{1}^{T} \mathbf{D}(\mathbf{u})
$$

em que $\mathbf{D}(u)$ é uma matriz diagonal $(n \times n)$ com elementos, dados por $\mathbf{u}=\left(u_{1}, \ldots, u_{n}\right)^{T}$ $\operatorname{com} u_{i}=\left[\psi\left(\phi+y_{i}\right)-\psi(\phi)-\frac{\left(y_{i}+\phi\right)}{\left(\phi+\mu_{i}\right)}+\log \left\{\frac{\phi}{\left(\phi+\mu_{i}\right)}\right\}+1\right]$ e 1 é um vetor $(n \times 1)$ de uns.

Alcançamos, assim, a matriz necessária para a obtenção da matriz de cuvartura normal para este esquema de perturbação.

\section{C.2 Pertubação aditiva em uma variável contínua do mo- delo}

Nesta seção, consideramos uma perturbação aditiva em uma variável contínua do modelo. Assim, supomos que a $r$-ésima variável explicativa fique expressa por $x_{\delta_{i}}^{(r)}=x_{i}^{(r)}+\delta_{i}, i=$ $1, \ldots, n$. Para este caso, temos

$$
g\left(\mu_{i}\right)=\mathbf{x}_{i}^{T} \boldsymbol{\beta}+\delta_{i} \beta_{r}+\mathbf{n}_{i}^{T} \mathbf{f} .
$$


Logo, obtemos as seguintes derivadas:

$$
\begin{aligned}
\boldsymbol{\Delta}_{1 j i} & =\frac{\partial^{2} \mathrm{~L}_{p}(\boldsymbol{\theta}, \lambda \mid \boldsymbol{\delta})}{\partial \beta_{j} \partial \delta_{i}} \\
& =\frac{\partial}{\partial \delta_{i}} w_{i} f_{i}^{*-1}\left(y_{i}-\mu_{i}\right) x_{i j} \\
& =w_{i} f_{i}^{*-1} x_{i j} \frac{\partial}{\partial \delta_{i}}\left(y_{i}-\mu_{i}\right) \\
& =-w_{i} f_{i}^{*-1} x_{i j} f_{i}^{*} \beta_{r} \\
& =-w_{i} x_{i j} \beta_{r}
\end{aligned}
$$

com forma matricial dada por

$$
\boldsymbol{\Delta}_{1}=-\beta_{r} \mathbf{X}^{T} \mathbf{W}
$$

Equivalentemente, para o parâmetro f , escrevemos da seguinte maneira:

$$
\begin{aligned}
\boldsymbol{\Delta}_{1 j i} & =\frac{\partial^{2} \mathrm{~L}_{p}(\boldsymbol{\theta}, \lambda \mid \boldsymbol{\delta})}{\partial \beta_{j} \partial \delta_{i}} \\
& =\frac{\partial}{\partial \delta_{i}} w_{i} f_{i}^{*-1}\left(y_{i}-\mu_{i}\right) n_{i j}-\frac{\partial}{\partial \delta_{i}}[\lambda \mathbf{K f}]_{j} \\
& =w_{i} f_{i}^{*-1} n_{i j} \frac{\partial}{\partial \delta_{i}}\left(y_{i}-\mu_{i}\right) \\
& =-w_{i} f_{i}^{*-1} n_{i j} f_{i}^{*} \beta_{r} \\
& =-w_{i} n_{i j} \beta_{r}
\end{aligned}
$$

com forma matricial dada por

$$
\Delta_{2}=-\beta_{r} \mathbf{N}^{T} \mathbf{W}
$$

Finalmente para $\phi$ temos

$$
\begin{aligned}
\boldsymbol{\Delta}_{3 i} & =\frac{\partial^{2} \mathrm{~L}_{p}(\boldsymbol{\theta}, \lambda \mid \boldsymbol{\delta})}{\partial \phi \partial \delta_{i}} \\
& =\frac{\partial}{\partial \delta_{i}} \psi\left(\phi+y_{i}\right)-\psi(\phi)-\frac{\left(y_{i}+\phi\right)}{\left(\phi+\mu_{i}\right)}+\log \left\{\frac{\phi}{\left(\phi+\mu_{i}\right)}\right\}+1 \\
& =\frac{\partial}{\partial \delta_{i}} \log \left\{\frac{\phi}{\left(\phi+\mu_{i}\right)}\right\} \\
& =-\beta_{r} f_{i}^{*}\left\{\left(\phi+y_{i}\right)+\frac{1}{\left(\phi+\mu_{i}\right)}\right\}
\end{aligned}
$$

com forma matricial dada por

$$
\boldsymbol{\Delta}_{3}=\mathbf{1}^{T} \mathbf{D}(\mathbf{s})
$$

em que $\mathbf{D}(\mathbf{s})$ é uma matriz diagonal $(n \times n)$ com elementos dados por $\mathbf{s}=\left(s_{1}, \ldots, s_{n}\right)^{T}$, 
$s_{i}=-\beta_{r} f_{i}\left\{\left(\phi+y_{i}\right)+\frac{1}{\left(\phi+\mu_{i}\right)}\right\}$.

Alcançamos, assim, a matriz necessária para a obtenção da matriz de cuvartura normal para este esquema de perturbação. 


\section{Apêndice D}

\section{Códigos em R}

\section{D.1 Ajuste do modelo via gamlss}

Carregue o pacote gamlss através do comando library (gamlss).

ajg.ozone<-gamlss (03 humidity+temp+ibh+ibt+vis+cs (doy, df=5),

family=NBI (mu.link = "log", sigma.link = "log"), data=ozone) summary (ajg.ozone)

\section{D.2 Gráfico envelope para o MSRBN ajustado via gamlss}

Para rodar este programa deixe no objeto fit . model a saída do ajuste do MSRBN ajustado via gamlss. Deixe também os dados disponíveis através do comando attach (...). A saída será o gráfico de envelope para o resíduo componente do desvio.

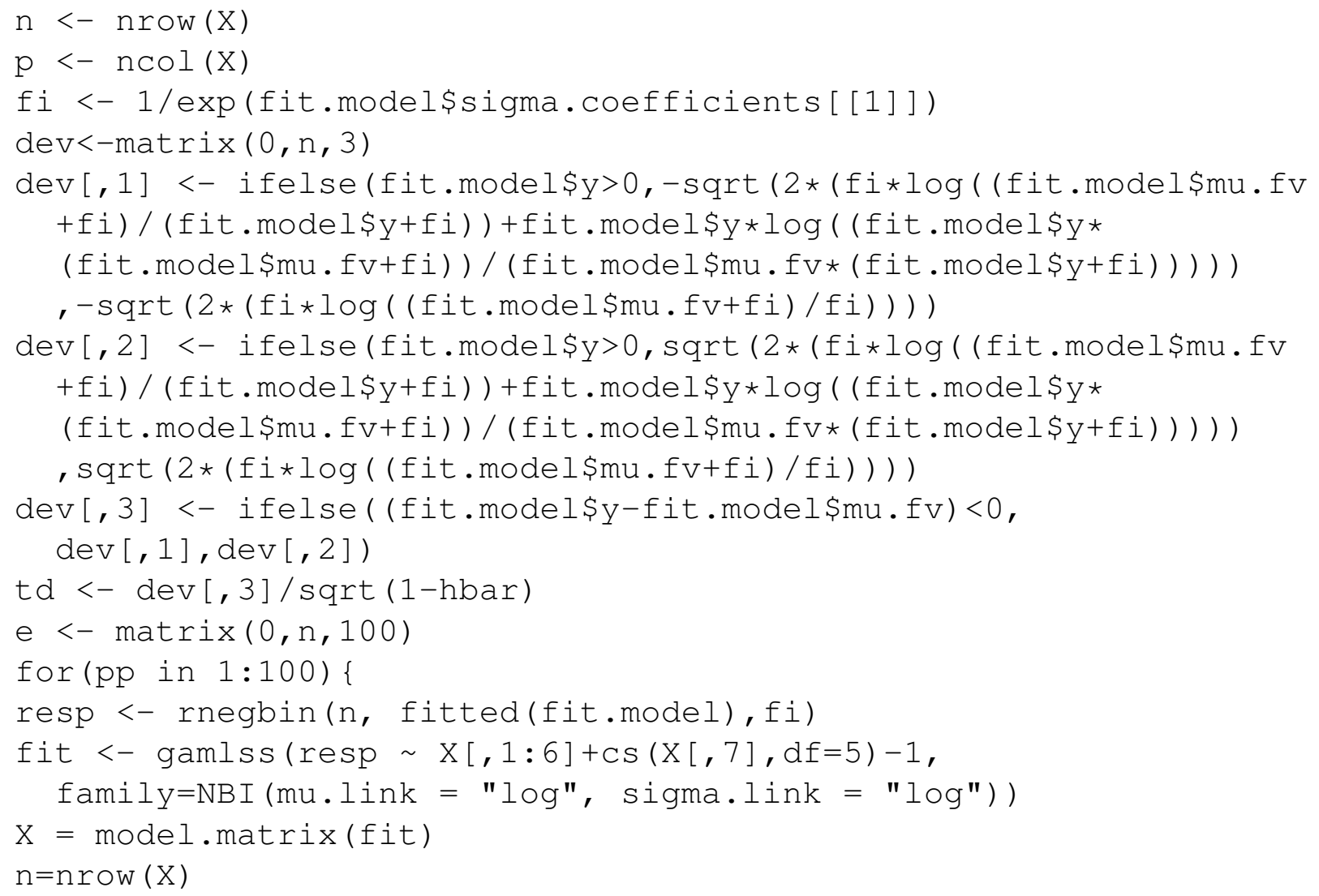




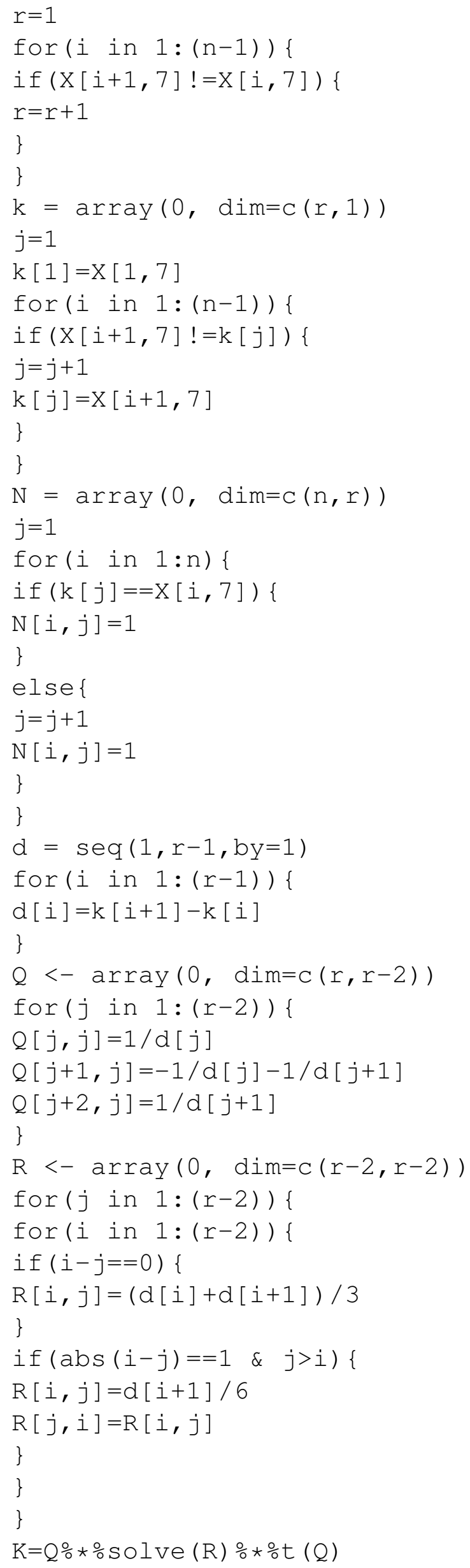




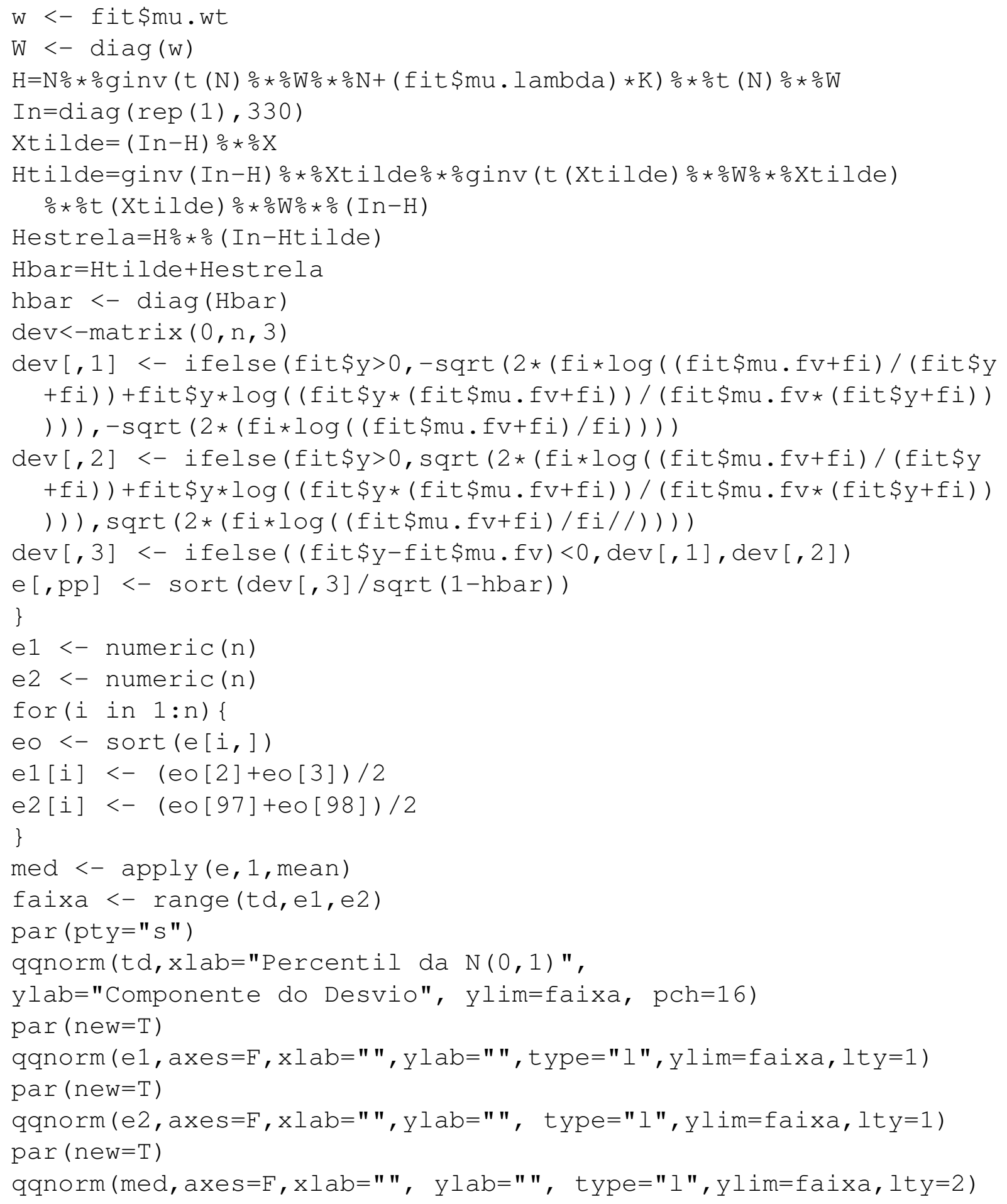

\section{D.3 Gráficos de diagnóstico para o MSRBN ajustado via gamlss}

Para rodar este programa deixe no objeto $\mathrm{f} i t$. model a saída do ajuste do MSRBN ajustado via gamlss. Deixe também os dados disponíveis através do comando attach ( . . . .

$\mathrm{X}=$ model.matrix (fit.model)

$\mathrm{n}=\operatorname{nrow}(\mathrm{X})$

$r=1$

for $(i$ in $1:(n-1))\{$ 


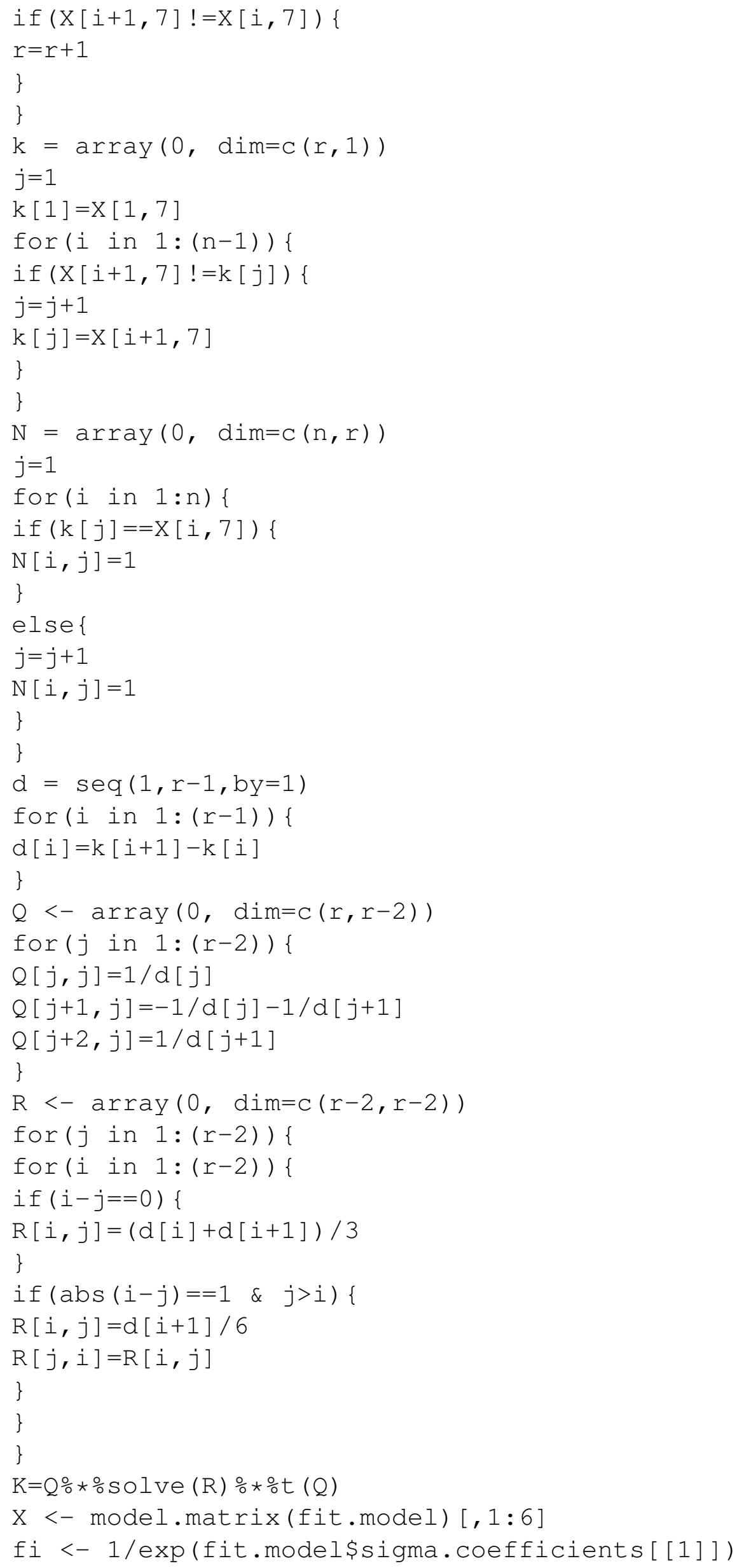




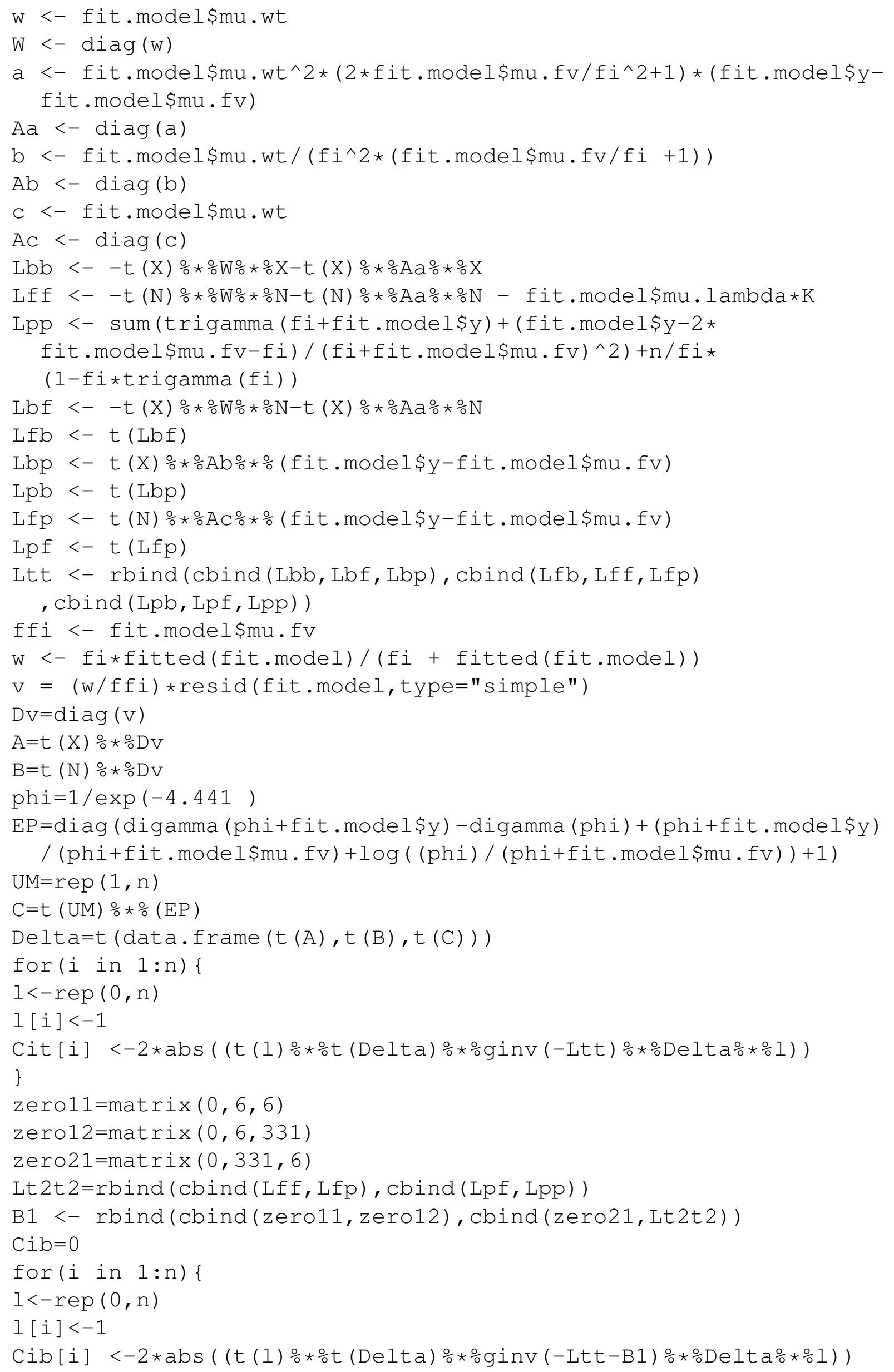


\}

zerol1=matrix $(0,329,329)$

zero12=matrix $(0,329,7)$

zero21=matrix $(0,7,329)$

Ltt $=r b i n d(c b i n d(L f f, L f b, L f p)$, cbind (Lbf, Lbb, Lbp)

, cbind (Lpf, Lpb, Lpp))

Lt 2t2=rbind (cbind (Lbb, Lbp), cbind ( Lpb, Lpp))

B1 <- rbind (cbind (zero11, zero12), cbind(zero21, Lt2t2))

Cif $=0$

for $(i$ in $1: n)\{$

$1<-\operatorname{rep}(0, \mathrm{n})$

$1[i]<-1$

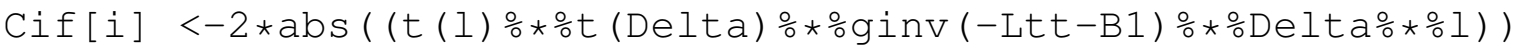
\}

zero11=matrix $(0,336,336)$

zero12=matrix $(0,1,336)$

zero $21=$ matrix $(0,336,1)$

Ltt $=r b i n d(c b i n d(L p p, L p b, L p f), c b i n d(L b p, L b b, L b f)$

, cbind (Lfp, Lfb, Lff))

Lt2t2=rbind (cbind (Lbb, Lbf), cbind (Lfb, Lff))

B1 <- rbind(cbind (zero11, zero12), cbind(zero21, Lt2t2))

$\mathrm{Cip}=0$

for $(\mathrm{i}$ in $1: \mathrm{n})\{$

$1<-r e p(0, n)$

$1[i]<-1$

Cip $[i]<-2 * \operatorname{abs}\left(\left(t(1) \div * \frac{\circ}{\circ}\right.\right.$ (Delta) $\left.\left.\% * g i n v(-\operatorname{Ltt}-\mathrm{B} 1) \div * \frac{\circ}{\circ} \operatorname{Delta} * \% 1\right)\right)$ \} 


\section{Referências Bibliográficas}

Akaike (1974) Hirotugu Akaike. A new look at the statistical model identification. IEEE Transactions on Automatic Control, 19(6):716-723. Citado na(s) pág(s). 16, 28

Breiman e Friedman (1985) Leo Breiman e Jerome H Friedman. Estimating optimal transformations for multiple regression and correlation. Journal of the American Statistical Association, 80(391):580-598. Citado na(s) pág(s). 26

Buja et al. (1989) Andreas Buja, Trevor Hastie e Robert Tibshirani. Linear smoothers and additive models. The Annals of Statistics, 17(2):453-510. Citado na(s) pág(s). 26

Cook (1977) R Dennis Cook. Detection of influential observation in linear regression. Technometrics, 19(1):15-18. Citado na(s) pág(s). 16, 30

Cook (1986) R Dennis Cook. Assessment of local influence. Journal of the Royal Statistical Society, Series B, 48(2):133-169. Citado na(s) pág(s). 30, 34

De Boor (1977) Carl De Boor. Package for calculating with b-splines. SIAM Journal on Numerical Analysis, 14(3):441-472. Citado na(s) pág(s). 20

De Boor (1978) Carl De Boor. A Practical Guide to Splines. Springer Verlag. Citado na(s) pág(s). 20

Dierckx (1993) Paul Dierckx. Curve and Surface Fitting with Splines. Oxford University Press, Inc. Citado na(s) pág(s). 20

Eilers e Marx (1996) Paul HC Eilers e Brian D Marx. Flexible smoothing with b-splines and penalties. Statistical Science, 11(2):89-102. Citado na(s) pág(s). 21

Faraway (2006, Cap. 12) Julian J Faraway. Extending the Linear Model with R: Generalized Linear, Mixed Effects and Nonparametric Regression Models. CRC Press. Citado na(s) pág(s). 2, 9,45

Green e Silverman (1994) Peter J Green e Bernard W Silverman. Nonparametric Regression and Generalized Linear Models: a Roughness Penalty Approach. Chapman \& Hall. Citado na(s) pág(s). 17, 21, 27

Green et al. (1985) Peter J Green, Christopher Jennison e Allan Seheult. Analysis of field experiments by least squares smoothing. Journal of the Royal Statistical Society. Series $B$ (Methodological), 47(2):299-315. Citado na(s) pág(s). 26

Lawless (1987) Jerald F Lawless. Negative binomial and mixed poisson regression. Canadian Journal of Statistics, 15(3):209-225. Citado na(s) pág(s). 13, 53 
Li (2010) Chin-Shang Li. Semiparametric negative binomial regression models. Communications in Statistics - Simulation and Computation@ $\AA$, 39(3):475-486. Citado na(s) pág(s). 17

Montgomery et al. (2001, Capítulo 7) Douglas C Montgomery, Elizabeth A Peck e G Geoffrey Vining. Introduction to Linear Regression Analysis. John Wiley \& Sons. Citado na(s) pág(s). 18

Noda (2013, Cap. 3) Gleyce Rocha Noda. Análise de Diagnóstico em Modelos Semiparamétricos Normais. Dissertação de Mestrado, Universidade de São Paulo. Citado na(s) pág(s). $27,31,32,37$

O'Sullivan (1986) Finbarr O'Sullivan. A statistical perspective on ill-posed inverse problems. Statistical Science, 1(4):502-518. Citado na(s) pág(s). 21

Paula (2013) Gilberto Alvarenga Paula. Modelos de Regressão: Com Apoio Computacional. IME-USP São Paulo. Citado na(s) pág(s). 5

R Core Team (2013) R Core Team. R: A Language and Environment for Statistical Computing. R Foundation for Statistical Computing, Vienna, Austria, 2013. URL http: //www.R-project.org/. Citado na(s) pág(s). 4, 5, 39

Reinsch (1967) Christian H Reinsch. Smoothing by spline functions. Numerische mathematik, 10(3):177-183. Citado na(s) pág(s). 20

Rigby e Stasinopoulos (2005) RA Rigby e DM Stasinopoulos. Generalized additive models for location, scale and shape. Journal of the Royal Statistical Society: Series C (Applied Statistics), 54(3):507-554. Citado na(s) pág(s). 17

Ruppert et al. (2003) David Ruppert, Matt P Wand e Raymond J Carroll. Semiparametric Regression. Cambridge University Press. Citado na(s) pág(s). 21

Schwarz (1978) Gideon Schwarz. Estimating the dimension of a model. The Annals of Statistics, 6(2):461-464. Citado na(s) pág(s). 16, 28

Svetliza e Paula (2003) Carolina F Svetliza e Gilberto A Paula. Diagnostics in nonlinear negative binomial models. Communications in Statistics-Theory and Methods, 32(6):12271250. Citado na(s) pág(s). 15

Svetliza (2002) Carolina Fabiana Svetliza. Modelos Não-lineares com Resposta Binomial Negativa. Tese de Doutorado, Instituto de Matemática e Estatística, Universidade de São Paulo, Brasil. Citado na(s) pág(s). 15, 16

Wang (1985) P. C. Wang. Adding a variable in generalized linear models. Technometrics, 27(3):273-276. Citado na(s) pág(s). 47

Wood (2006, Cap. 4) Simon Wood. Generalized Additive Models: an Introduction with $R$. CRC press. Citado na(s) pág(s). 28 